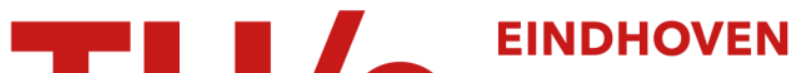 \\ UNIVERSITY OF \\ TECHNOLOGY
}

\section{Fractional evolution equations in Banach spaces}

\section{Citation for published version (APA):}

Bazhlekova, E. G. (2001). Fractional evolution equations in Banach spaces. [Phd Thesis 1 (Research TU/e / Graduation TU/e), Mathematics and Computer Science]. Technische Universiteit Eindhoven.

https://doi.org/10.6100//R549476

DOI:

10.6100/IR549476

Document status and date:

Published: 01/01/2001

\section{Document Version:}

Publisher's PDF, also known as Version of Record (includes final page, issue and volume numbers)

\section{Please check the document version of this publication:}

- A submitted manuscript is the version of the article upon submission and before peer-review. There can be important differences between the submitted version and the official published version of record. People interested in the research are advised to contact the author for the final version of the publication, or visit the $\mathrm{DOI}$ to the publisher's website.

- The final author version and the galley proof are versions of the publication after peer review.

- The final published version features the final layout of the paper including the volume, issue and page numbers.

Link to publication

\section{General rights}

Copyright and moral rights for the publications made accessible in the public portal are retained by the authors and/or other copyright owners and it is a condition of accessing publications that users recognise and abide by the legal requirements associated with these rights.

- Users may download and print one copy of any publication from the public portal for the purpose of private study or research.

- You may not further distribute the material or use it for any profit-making activity or commercial gain

- You may freely distribute the URL identifying the publication in the public portal.

If the publication is distributed under the terms of Article $25 \mathrm{fa}$ of the Dutch Copyright Act, indicated by the "Taverne" license above, please follow below link for the End User Agreement:

www.tue.nl/taverne

Take down policy

If you believe that this document breaches copyright please contact us at:

openaccess@tue.nl

providing details and we will investigate your claim. 


\title{
Fractional Evolution Equations in Banach Spaces
}

\author{
PROEFSCHRIFT \\ ter verkrijging van de graad van doctor aan de \\ Technische Universiteit Eindhoven, op gezag van de \\ Rector Magnificus, prof.dr. R.A. van Santen, voor een \\ commissie aangewezen door het College voor \\ Promoties in het openbaar te verdedigen \\ op maandag 1 oktober 2001 om 16.00 uur
}

door

Emilia Grigorova Bajlekova

geboren te Pleven, Bulgarije 
Dit proefschrift is goedgekeurd door de promotoren:

prof.dr.ir. J. de Graaf

en

prof.dr. Ph.P.J.E. Clément

This thesis was published by University Press Facilities Eindhoven University of Technology 
Dedicated to the memory of my mother in law Svetla 



\section{Preface}

In recent years a considerable interest has been shown in the so-called fractional calculus, which allows us to consider integration and differentiation of any order, not necessarily integer. To a large extent this is due to the applications of the fractional calculus to problems in different areas of physics and engineering.

The fractional calculus can be considered an old and yet novel topic. Starting from some speculations of Leibniz and Euler, followed by the works of other eminent mathematicians including Laplace, Fourier, Abel, Liouville and Riemann, it has undergone a rapid development especially during the past two decades. One of the emerging branches of this study is the theory of fractional evolution equations, i.e. evolution equations where the integer derivative with respect to time is replaced by a derivative of fractional order. The increasing interest in this class of equations is motivated both by their application to problems from viscoelasticity, heat conduction in materials with memory, electrodynamics with memory, and also because they can be employed to approach nonlinear conservation laws.

This thesis is concerned with abstract fractional evolution equations. It is an outcome of the author's research during her Ph.D. study at the Eindhoven University of Technology (December 1997 - September 2001). Most of the material in the thesis is based on the following articles from this period:

[1] E. Bazhlekova, The abstract Cauchy problem for the fractional evolution equation. Fractional Calculus 85 Applied Analysis 1, No 3 (1998), 255-270.

[2] E. Bazhlekova, Perturbation properties for abstract evolution equations of fractional order. Transform Methods and Special Functions, AUBG'99. Fractional Calculus \& Applied Analysis 2, No 4 (1999), 359-366.

[3] E. Bazhlekova, Subordination principle for fractional evolution equations. Fractional Calculus $\&$ Applied Analysis 3, No 3 (2000), 213-230.

[4] E. Bazhlekova, Perturbation and approximation properties for abstract evolution equations of fractional order, Research Report RANA 00-05, Eindhoven University of Technology, Eindhoven (2000).

[5] E. Bazhlekova, Maximal $L^{p}$ regularity of fractional order equations, preprint.

[6] Ph. Clément, E. Bazhlekova, Global solutions for a quasilinear fractional evolution equation, in preparation. 
Chapter 1 contains notations and some background material. In Chapter $2([\mathbf{1}],[\mathbf{2}],[4])$ applying functional analytical methods, the solvability of the problem and the properties of the solution operator are investigated. We develop a theory which extends the classical theory of $C_{0}$-semigroups of operators. The subordination principlewe is studied in detail in Chapter 3 ([3]) applying transform methods. Chapter 4 ([5]) is devoted to the maximal $L^{p}$ regularity. In Chapter 5 the problem of regularity is tackled in Hilbert space settings by the method of sums of accretive operators. The obtained regularity results are applied in Chapter $6([6])$ to investigate the nonautonomous fractional evolution equations, a transient case between linear and quasilinear problems. As an application, the global solvability of a quasilinear fractional evolution problem is obtained.

Here I would like to thank all the people, who helped me during the preparation of the thesis. I am grateful to prof.dr.ir. J. de Graaf, who gave me the opportunity to carry out my doctorate research in the group of Applied Analysis at the Eindhoven University of Technology, for many helpful discussions and constant encouragement. I am indebted to prof.dr. Ph. Clément for keeping me up to date with the very recent developments of the topic, for his inspiring suggestions, criticism and patience. I am grateful to prof.dr. V. Kiryakova for introducing me to the world of fractional calculus. Thanks are also due to prof.dr.ir. M.L.J. Hautus and prof.dr. J. Boersma for the critical reading of parts of the manuscript. I thank the members of the Applied Analysis group for providing a pleasant and stimulating working environment. Last but not least I thank my husband Ivan and our sons Svetlio and Galin for their help, patience and understanding. 


\section{Contents}

$\begin{array}{ll}\text { Introduction } & 1\end{array}$

1 Fractional integration and differentiation 5

1.1 Preliminaries . . . . . . . . . . . . . . . . . . 5

1.2 Fractional integration and differentiation f........... 10

1.3 Mittag-Leffler and Wright functions . . . . . . . . . . . . . 12

1.4 Operators of fractional differentiation in $\boldsymbol{L}^{p}$ spaces . . . . . . . . 14

2 Solution operators: generation, approximation and perturbation $\quad 19$

2.1 Solution operators . . . . . . . . . . . . . . . . . . . . 19

2.2 Analytic solution operators . . . . . . . . . . . 25

2.3 Approximation . . . . . . . . . . . . . . . . 29

2.4 Perturbation properties . . . . . . . . . . . 33

3 Subordination principle $\quad 39$

3.1 Subordination principle and its invertibility . . . . . . . . . . 39

3.2 An inversion formula . . . . . . . . . . . . . . . 45

3.3 The semigroup of subordination operators . . . . . . . . . 48

$4 \quad$ Strict $L^{p}$ solvability

4.1 The notion of maximal $\boldsymbol{L}^{p}$ regularity . . . . . . . . . . . 53

4.2 Maximal $\boldsymbol{L}^{p}$ regularity for fractional order equations . . . . . . . 55

4.3 Strict $\boldsymbol{L}^{\boldsymbol{p}}$ solutions of fractional order equations . . . . . . . . 61

$5 \quad$ Maximal regularity in Hilbert spaces via accretivity $\quad 71$

5.1 Two classical results . . . . . . . . . . . . . . . . 71

5.2 The case of nonlinear $\boldsymbol{m}$-accretive operators with $\boldsymbol{\alpha} \in(\mathbf{0 , 1}) \ldots 72$ 
5.3 Reduction of problems with $\alpha \in(\mathbf{1}, \mathbf{2}) \ldots \ldots \ldots 76$

5.4 Linear nonautonomous problems with $\alpha \in(\mathbf{0 , 1}) \ldots \ldots$. . . . 80

5.5 Example: fractional Löwner-Kufarev equation . . . . . . . . . 83

6 Quasilinear problems $\quad 87$

6.1 Linear nonautonomous case . . . . . . . . . . . . 87

6.2 Global solutions for a quasilinear equation . . . . . . . . . . 93

$\begin{array}{lr}\text { Bibliography } & 99\end{array}$

$\begin{array}{ll}\text { Index } & 105\end{array}$

$\begin{array}{ll}\text { Curriculum Vitae } & 107\end{array}$ 


\section{Introduction}

This thesis is devoted to the study of abstract differential equations of fractional order, describing the evolution in time of the state of a system.

Let $\alpha>0$ and $m=\lceil\alpha\rceil$, the smallest integer greater than or equal to $\alpha$. Assume $u:[0, \infty) \rightarrow X$, where $X$ is a Banach space. Define the fractional derivative of $u$ of order $\alpha$ by

$$
D_{t}^{\alpha} u(t):=\frac{d^{m}}{d t^{m}} \int_{0}^{t} g_{m-\alpha}(t-s) u(s) d s, t>0
$$

where

$$
g_{\beta}(t):=\frac{t^{\beta-1}}{\Gamma(\beta)}, \quad t>0, \beta \geq 0 .
$$

When $\alpha=n$ is integer, we set $D_{t}^{n}:=\frac{d^{n}}{d t^{n}}, n=1,2, \ldots$.

Let $A$ be a closed linear operator densely defined in $X$. Given $x \in X$, we investigate first the following problem:

$$
D_{t}^{\alpha}(u-x)=A u(t), \quad t>0,
$$

with initial conditions $u(0)=x, u^{(k)}(0)=0, k=1,2, \ldots, m-1$. If $A u(t)$ is continuous in $t$ (this we require later in the definition of strong solution), the initial conditions are implied automatically by the equation (1).

In the case $\alpha=1$ this problem coincides with the classical abstract Cauchy problem of first order

$$
u^{\prime}(t)=A u(t), \quad t>0 ; \quad u(0)=x .
$$

There is a vast amount of literature devoted to it and its equivalent formulation - the semigroup theory. Choosing $\alpha=2$ results in the second-order problem

$$
u^{\prime \prime}(t)=A u(t), \quad t>0 ; u(0)=x, u^{\prime}(0)=0,
$$

with the corresponding theory leading to the concept of a cosine family. The study of problem (1) presented in this thesis (Chapters 2 and 3) is thus an extension (of some ideas) of the semigroup theory and the theory of cosine families.

Let $\mathbb{R}_{+}:=[0, \infty)$. A function $u \in C\left(\mathbb{R}_{+} ; X\right)$ is called a strong solution of (1) if $u \in C\left(\mathbb{R}_{+} ; D(A)\right) \cap C^{m-1}\left(\mathbb{R}_{+} ; X\right), g_{m-\alpha} *(u-x) \in C^{m}\left(\mathbb{R}_{+} ; X\right)$ and $(1)$ holds on $\mathbb{R}_{+}$. The concept of well-posedness of $(1)$ is a direct extension of the corresponding notion 
usually employed for the abstract Cauchy problem (2). For well-posed problems we define $S_{\alpha}(t) x:=u(t)$, where $u(t)$ is the solution of (1), and call the operator function $S_{\alpha}(t)$ the solution operator of $(1)$.

Note that in case of noninteger $\alpha$ there is no analogue of the semigroup property

$$
S_{1}(t+s)=S_{1}(t) S_{1}(s)
$$

or cosine functional equation

$$
S_{2}(t+s)+S_{2}(t-s)=2 S_{2}(t) S_{2}(s),
$$

which play a crucial role in the developing of the corresponding theories. This is due to the nonlocal character of the fractional differentiation leading always to some presence of memory.

Equation (1) (possibly with a forcing function $f(t)$ ) can be rewritten in an equivalent form as

$$
u(t)=\int_{0}^{t} g_{\alpha}(t-s) A u(s) d s+h(t), \quad t>0,
$$

where $h(t):=x+\int_{0}^{t} g_{\alpha}(t-s) f(s) d s$. So, we can rely in our study on the basic results on abstract Volterra integral equations. A very rich source is the monograph on evolutionary integral equations [64].

The representation (4) shows clearly the causality of the system described, i.e. the present state of the system is determined only by its history and the present force, but does not depend on the future.

The generation, approximation and perturbation results presented in Chapter 2 generalize some facts concerning $C_{0}$-semigroups and cosine families, but also distinguish some new features. The analogue of the Hille-Yosida theorem (see [25]) reads as follows: The problem (1) is well-posed with solution operator $S_{\alpha}(t)$, satisfying $\left\|S_{\alpha}(t)\right\| \leq M e^{\omega t}$ for some $M, \omega>0$, if and only if $\left(\omega^{\alpha}, \infty\right)$ belongs to the resolvent set of $A$ and

$$
\left\|\frac{\partial^{n}}{\partial \lambda^{n}}\left(\lambda^{\alpha-1}\left(\lambda^{\alpha}-A\right)^{-1}\right)\right\| \leq \frac{M n !}{(\lambda-\omega)^{n+1}}, \lambda>\omega, n=0,1,2, \ldots
$$

This criterion, however, is practically not applicable because it requires verification of infinitely many inequalities. This motivates the study of other aspects of problem (1) such as generation of analytic solution operators, subordination, perturbation, giving other methods to prove well-posedness.

The obtained representation formula for the solution operator $S_{\alpha}(t)$, which is a generalization of the exponential formula for $C_{0}$-semigroups $S_{1}(t) x=\lim _{n \rightarrow \infty}\left(I-\frac{t}{n} A\right)^{-n} x, x \in$ $X, t \geq 0$, is important for the numerical approximation of the solution, especially for implicit approximation schemes.

Another aspect of our study is the relationship between problems (1) with different orders $\alpha$, resulting in the so-called subordination principle (Chapter 3). This is an extension of the abstract Weierstrass formula relating the semigroup $S_{1}$ and the cosine family $S_{2}$,

$$
S_{1}(t)=\frac{1}{\sqrt{\pi t}} \int_{0}^{\infty} e^{-s^{2} /(4 t)} S_{2}(s) d s, t>0
$$


which shows that if $A$ generates a cosine family, it is necessarily a generator of an analytic semigroup of angle $\pi / 2$. Roughly speaking, the subordination principle means that one and the same operator $A$ guarantees better properties of the solution if $\alpha$ is smaller and that the set of operators $A$, such that (1) is well-posed, shrinks when $\alpha$ increases. In particular, if $\alpha>2$, then $A$ is necessarily bounded. For this reason we consider mostly $\alpha \in(0,2)$. The subordination principle is studied in detail, involving the problems of invertibility and the properties of the related semigroup of subordination operators.

The next two chapters are devoted to the regularity aspects of the fractional evolution equations. Maximal regularity of a problem with a forcing function $f$ means that $A u$ has the same "smoothness" as $f$, which is in principle not always the case.

Let $\alpha \in(0,2), m=\lceil\alpha\rceil, 1<p<\infty$ and $T>0$. Let $A$ be a closed positive linear operator of $D(A) \subset X$ into $X$. Instead of working with continuous functions of time we work now with functions which are of class $L^{p}$ and consider the problem

$$
\begin{aligned}
& D_{t}^{\alpha} u(t)+A u(t)=f(t), \quad \text { a.a. } t \in[0, T], \\
& \left(g_{1-\alpha} * u\right)(0)=x_{0}, \quad \text { if } \alpha \in(0,1), \\
& \left(g_{2-\alpha} * u\right)(0)=x_{0}, \quad\left(g_{2-\alpha} * u\right)^{\prime}(0)=x_{1}, \quad \text { if } \alpha \in(1,2) .
\end{aligned}
$$

Let first $x_{0}=x_{1}=0$ and define the operators $\mathcal{A}$ and $\mathcal{L}_{\alpha}$ by

$$
\begin{array}{ll}
(\mathcal{A} u)(t):=(A u)(t), & D(\mathcal{A}):=L^{p}(0, T ; D(A)) \\
\left(\mathcal{L}_{\alpha} u\right)(t):=\left(D_{t}^{\alpha} u\right)(t), & D\left(\mathcal{L}_{\alpha}\right):=\left\{u \in L^{p}(0, T ; X) \mid g_{m-\alpha} * u \in W_{0}^{m, p}(0, T ; X)\right\} .
\end{array}
$$

The properties of operators $\mathcal{L}_{\alpha}$ and their domains of definition are studied in Chapter 1 . We say that there is maximal $L^{p}$ regularity of problem (5) if for every $f \in L^{p}(0, T ; X)$ there exists a unique $u \in D(\mathcal{A}) \cap D\left(\mathcal{L}_{\alpha}\right)$ satisfying $(5)$.

This problem can be reformulated as the operator equation

$$
\mathcal{L}_{\alpha} u+\mathcal{A} u=f
$$

One then can apply the method of sums to study (6). To this aim the theorems of Da Prato-Grisvard and Dore-Venni or some very recent results involving the concept of $\mathcal{R}$-boundedness can be used. They imply that (5) with $x_{0}=x_{1}=0$ has maximal $L^{p}$ regularity if and only if the family of operators $(i \rho)^{\alpha}\left((i \rho)^{\alpha} I+A\right)^{-1}, \rho \in(-\infty, 0) \cup(0,+\infty)$, is $\mathcal{R}$-bounded. Since this is a very recent topic, a part of the references have not been published yet.

Concerning the equation with $f=0, x_{0}, x_{1} \in X$, a direct approach is applied to study problem (5). It appears that the solution $u \in L^{p}(0, T ; D(A))$, whenever $x_{0}$ and $x_{1}$ belong to some real interpolation spaces. Combining the above two cases we obtain conditions on $A, x_{0}$ and $x_{1}$, under which, for any $f \in L^{p}(0, T ; X)$, problem $(5)$ has a strict $L^{p}$ solution, i.e. a solution satisfying $A u \in L^{p}(0, T ; X)$ (Chapter 4).

A word explaining the change of the form of the equation (1) to (5) seems to be necessary. Assume $\alpha \in(0,1)$. In the case of continuous functions, $u(0)$ is well defined and the form (1), which yields an initial condition $u(0)=x$, is more natural. In the case of $L^{p}$ functions 
it is known that the elements of $D\left(\mathcal{L}_{\alpha}\right)$ are continuous only if $\alpha>1 / p$. So, it is more appropriate to give initial values not to the function $u$ itself, but to $g_{1-\alpha} * u$, which is always continuous for $u \in D\left(\mathcal{L}_{\alpha}\right)$. The operator $A$ is replaced by $-A$ because the form (6) is more natural to apply the theory of sums of operators. Let us note that problem $(5)$ can be rewritten in the form (4) with $A$ replaced by $-A$ and $h(t)=\int_{0}^{t} g_{\alpha}(t-s) f(s) d s+g(t)$ where $g(t)=x_{0} g_{\alpha}(t)$ if $\alpha \in(0,1)$ and $g(t)=x_{0} g_{\alpha-1}(t)+x_{1} g_{\alpha}(t)$ if $\alpha \in(1,2)$. This representation shows that the problems (1) and (5) are principally of the same form.

When we work in $L^{2}(0, T ; H)$, where $H$ is a Hilbert space (Chapter 5$)$, a different approach can be used to prove regularity: the method of sums of accretive operators. It is applicable even in the case when $A$ is nonlinear. Since $\mathcal{L}_{\alpha}$ is accretive only for $\alpha \in(0,1)$, this approach cannot be applied for $\alpha \in(1,2)$. To do this indirectly we use a result on reducing an equation of order $\alpha$ to a corresponding equation of order $\alpha / 2$. This is an approach providing a simple proof of regularity, but without further development it can be applied only for a quite limited class of operators $A$. Nonautonomous problems in Hilbert space settings are also tackled by the theory of sums of accretive operators. Applications to the so-called fractional Löwner-Kufarev equation are presented.

Nonautonomous problems are important especially as a transient case between the linear and the nonlinear theory. General results on nonautonomous equations in Banach spaces are obtained in Chapter 6 on the basis of the regularity theorems of the corresponding autonomous problems using an inductive argument in time. These results are applied to obtain the global solvability of the following quasilinear fractional evolution equation of order $\alpha \in(1,2)$, intermediate to the quasilinear diffusion equation and quasilinear wave equation:

$$
D_{t}^{\alpha} u=\left(\sigma\left(u_{x}\right)\right)_{x}+f .
$$

Here $u=u(t, x)$ is a real-valued function for $t>0, x \in(0,1)$, and the subscript $x$ denotes the partial derivative with respect to $x$. The Dirichlet boundary condition

$$
u(t, 0)=u(t, 1)=0, t>0,
$$

and initial conditions

$$
\lim _{t \downarrow 0} \int_{0}^{t} g_{2-\alpha}(t-s) u(s, x) d s=0 ; \quad \lim _{t \downarrow 0} \frac{d}{d t} \int_{0}^{t} g_{2-\alpha}(t-s) u(s, x) d s=0,
$$

are assumed. We suppose that $\sigma \in C^{2}(-\infty,+\infty)$ and satisfies

$$
0<\sigma_{0} \leq \sigma^{\prime}(y) \leq \sigma_{1}<\infty, y \in(-\infty,+\infty)
$$

for some constants $\sigma_{0}, \sigma_{1}$. While the behaviour of the solution of (7) in the linear case $\sigma(y)=y$ is reasonably well understood, only very partial results exist for the nonlinear equations of this type. The existence of global smooth solutions in a very general setting is proved in [31] but only for $\alpha<4 / 3$. In [43] this is proved for the Hilbert space case under an assumption on the deviation of $\sigma$ from a linear function. We use a similar assumption and apply $L^{p}\left(L^{q}\right)$ estimates to establish global existence of strong solution of (7) in $L^{p}\left(0, T ; L^{q}(0,1)\right)$ for all $\alpha \in(1,2)$ and $p, q$ - sufficiently large. 


\section{Chapter 1}

\section{Fractional integration and differentiation}

This section contains some preliminaries used throughout the whole thesis. After presenting some notations and definitions, the operators of fractional integration and differentiation of Rieman-Liouville and Caputo type are defined. Next we introduce two special functions intimately related to fractional differential equations. At the end of this chapter we study the properties of the operator of Riemann-Liouville fractional differentiation in $L^{p}$ spaces which are important for the theory of maximal $L^{p}$ regularity developed in Chapter 4, but are also of independent interest.

\subsection{Preliminaries}

\section{Some notations}

Most notations used throughout this thesis are standard. So, $\mathbb{N}, \mathbb{R}, \mathbb{C}$ denote the sets of natural, real and complex numbers, respectively, and $\mathbb{N}_{0}:=\mathbb{N} \cup\{0\}, \mathbb{R}_{+}:=[0, \infty)$,

$$
\Sigma_{\theta}(\omega):=\{\lambda \in \mathbb{C} \backslash\{0\}|| \arg (\lambda-\omega) \mid<\theta\}, \theta \in[0, \pi), \omega \in \mathbb{R},
$$

and $\Sigma_{\theta}:=\Sigma_{\theta}(0)$ for short. If $\alpha>0,\lfloor\alpha\rfloor$ denotes the largest integer less than or equal to $\alpha$ and $\lceil\alpha\rceil$ denotes the smallest integer greater than or equal to $\alpha$.

Let $X, Y$ be Banach spaces with norms $\|.\|_{X},\|.\|_{Y}$; the subscripts will be dropped when there is no danger of confusion. By $\mathcal{B}(X, Y)$ we denote the space of all bounded linear operators from $X$ to $Y, \mathcal{B}(X):=\mathcal{B}(X, X)$ for short. If $A$ is a linear operator in $X$ then $D(A), R(A), N(A)$ denote domain, range and null space of $A$, respectively, while $\sigma(A)$ and $\varrho(A)$ mean spectrum and resolvent set of $A$ and $R(\lambda, A):=(\lambda I-A)^{-1}$ stands for the resolvent operator of $A$. We say that the Banach space $X$ is continuously embedded in the Banach space $Y$ and write

$$
X \hookrightarrow Y
$$

if $X \subset Y$ and $\|\cdot\|_{Y} \leq C\|\cdot\|_{X}$. 
Let $J=(a, b)$, where $-\infty \leq a<b \leq+\infty$, and $1 \leq p<\infty$. Then $L^{p}(J ; X)$ denotes the space of all (equivalent classes of) Bochner-measurable functions $f: J \rightarrow X$, such that $\|f(t)\|_{X}^{p}$ is integrable for $t \in J$. It is a Banach space when normed by

$$
\|f\|_{L^{p}(J ; X)}:=\left(\int_{J}\|f(s)\|_{X}^{p} d s\right)^{1 / p} .
$$

If $p=\infty$ the space $L^{p}(J ; X)$ consists of all measurable functions with a finite norm

$$
\|f\|_{L^{\infty}(J ; X)}:=\operatorname{ess} \sup _{t \in J}\|f(t)\|_{X} .
$$

If $X$ is the underlying scalar field $\mathbb{R}$ or $\mathbb{C}$, the image space in the function space notation introduced above and further in this section, will be dropped. For example we write $L^{p}(J)$ instead of $L^{p}(J ; \mathbb{R})$. As usual, * denotes the convolution of functions, defined on $\mathbb{R}$ or on $\mathbb{R}_{+}$:

$$
\begin{gathered}
(k * f)(t)=\int_{-\infty}^{+\infty} k(t-s) f(s) d s, \quad t \in \mathbb{R}, k \in L^{1}(\mathbb{R}), f \in L^{1}(\mathbb{R} ; X), \\
(k * f)(t)=\int_{0}^{t} k(t-s) f(s) d s, \quad t \in \mathbb{R}_{+}, k \in L^{1}\left(\mathbb{R}_{+}\right), f \in L^{1}\left(\mathbb{R}_{+} ; X\right) .
\end{gathered}
$$

We recall two important inequalities:

- the Hölder inequality:

$$
\int_{J}\|f(t) g(t)\|_{X} d t \leq\|f\|_{L^{p}(J ; X)}\|g\|_{L^{p^{\prime}(J ; X)}}
$$

where $p^{\prime}=p /(p-1)$ and $f \in L^{p}(J ; X), g \in L^{p^{\prime}}(J ; X)$. We see that the embedding $L^{p_{1}}(J ; X) \hookrightarrow L^{p_{2}}(J ; X), p_{1}>p_{2} \geq 1$, is derived from the Hölder inequality, if $J$ is a finite interval.

- the Young inequality: if $k \in L^{1}(J), f \in L^{p}(J ; X)$ for some $p \in[1, \infty)$, then $k * f \in$ $L^{p}(J ; X)$ and

$$
\|k * f\|_{L^{p}(J ; X)} \leq\|k\|_{L^{1}(J)}\|f\|_{L^{p}(J ; X)} .
$$

By $C(J ; X)$, resp. $C^{m}(J ; X)$, we denote the spaces of functions $f: J \rightarrow X$, which are continuous, resp. $m$-times continuously differentiable; $C_{0}^{\infty}(\mathbb{R} ; X)$ consists of all infinitely differentiable functions with compact support.

Let now $I=[0, T], T>0$. Then $C(I ; X)$ and $C^{m}(I ; X)$ are Banach spaces endowed with the norms

$$
\|f\|_{C}:=\sup _{t \in I}\|f(t)\|_{X}, \quad\|f\|_{C^{m}}:=\sup _{t \in I} \sum_{k=0}^{m}\left\|f^{(k)}(t)\right\|_{C} .
$$

The Hölder spaces $C^{\gamma}, 0<\gamma<1$, are defined by

$$
C^{\gamma}(I ; X):=\left\{f \in C(I ; X) \mid \sup _{s, t \in I} \frac{\|f(t)-f(s)\|_{X}}{|t-s|^{\gamma}}<\infty\right\}
$$


with

$$
\|f\|_{C^{\gamma}}:=\sup _{t \in I}\|f(t)\|_{X}+\sup _{s, t \in I} \frac{\|f(t)-f(s)\|_{X}}{|t-s|^{\gamma}} .
$$

The little Hölder spaces $h^{\gamma}$ are defined by

$$
h^{\gamma}(I ; X):=\left\{f \in C(I ; X) \mid \limsup _{s, t \in I, 0<|t-s| \leq \delta, \delta \downarrow 0} \frac{\|f(t)-f(s)\|_{X}}{|t-s|^{\gamma}}=0\right\} .
$$

If $\lambda>1$ we define $C^{\lambda}(I ; X)$ as the space of all functions satisfying $f \in C^{m}(I, X)$ and $f^{(m)} \in C^{\gamma}(I ; X)$, where $m=\lfloor\lambda\rfloor, \gamma=\lambda-m$. It is endowed with the norm

$$
\|f\|_{C^{\lambda}}=\|f\|_{C^{m}}+\left\|f^{(m)}\right\|_{C^{\gamma}} .
$$

Let $I=(0, T)$, or $I=\mathbb{R}_{+}$, or $I=\mathbb{R}, m \in \mathbb{N}, 1 \leq p<\infty$. The Sobolev spaces can be defined in the following way (see [8], Appendix):

$$
W^{m, p}(I ; X):=\left\{f \mid \exists \varphi \in L^{p}(I ; X): f(t)=\sum_{k=0}^{m-1} c_{k} \frac{t^{k}}{k !}+\frac{t^{m-1}}{(m-1) !} * \varphi(t), t \in I\right\} .
$$

Note that $\varphi(t)=f^{(m)}(t), c_{k}=f^{(k)}(0)$. Let

$$
W_{0}^{m, p}(I ; X):=\left\{f \in W^{m, p}(I ; X) \mid f^{(k)}(0)=0, k=0,1, \ldots, m-1\right\} .
$$

So, $f \in W_{0}^{m, p}(I ; X)$ iff $f=\frac{t^{m-1}}{(m-1) !} * \varphi$ for some $\varphi \in L^{p}(I ; X)$.

The Laplace transform of a function $f \in L^{1}\left(\mathbb{R}_{+} ; X\right)$ is defined by

$$
\widehat{f}(\lambda):=\int_{0}^{\infty} e^{-\lambda t} f(t) d t, \quad \operatorname{Re} \lambda>\omega
$$

if the integral is absolutely convergent for $\operatorname{Re} \lambda>\omega$.

The Fourier transform of a function $f \in L^{1}(\mathbb{R} ; X)$ is denoted by

$$
\widetilde{f}(\rho):=\int_{-\infty}^{\infty} e^{-i \rho t} f(t) d t, \quad \rho \in \mathbb{R}
$$

\section{Operators in Banach spaces}

Let $X$ be a complex Banach space, and let $A: D(A) \subset X \rightarrow X$ be a closed linear densely defined operator in $X$. In the sequel we suppose that $D(A)$ is equipped with the graph norm of $A$, i.e. $\|x\|_{D(A)}:=\|x\|_{X}+\|A x\|_{X}$; since $A$ is closed, $D(A)$ is a Banach space, continuously and densely embedded into $X$.

Definition 1.1 We call an operator $A: D(A) \subset X \rightarrow X$ nonnegative iff the following two conditions are satisfied: 
(i) there exists $K \geq 0$ such that for all $\lambda>0$ and all $u \in D(A)$,

$$
\lambda\|u\|_{X} \leq K\|\lambda u+A u\|_{X}
$$

holds;

(ii) $R(\lambda I+A)=X$ for all $\lambda>0$.

Observe that if $A$ satisfies (i) and (ii), it is closed. Moreover, any nonnegative operator in a reflexive Banach space is densely defined [50]. If $A$ is a nonnegative operator on $X$, then define

$$
\begin{gathered}
\phi_{A}:=\sup \left\{\phi \in[0, \pi] \mid \rho(-A) \supset \overline{\Sigma_{\phi}}, \sup _{\lambda \in \overline{\Sigma_{\phi}}}\left\|\lambda(\lambda I+A)^{-1}\right\|_{\mathcal{B}(X)}<\infty\right\} \\
K_{A}(\phi):=\sup _{\lambda \in \overline{\Sigma_{\phi}}}\left\|\lambda(\lambda I+A)^{-1}\right\|_{\mathcal{B}(X)}, \phi<\phi_{A} .
\end{gathered}
$$

The spectral angle of $A$ is defined by

$$
\omega_{A}:=\pi-\phi_{A}
$$

Definition 1.2 An operator A satisfying (i) of Definition 1.1 with $K=1$ is called accretive. If $A$ moreover satisfies (ii) then $A$ is called $\boldsymbol{m}$-accretive.

Note that if $A$ is accretive and (ii) holds for some $\lambda_{0}>0$ then it holds for all $\lambda>0$, so it is $m$-accretive. Indeed, the equation $\lambda u+A u=f$ is equivalent to

$$
u=\lambda_{0}\left(\lambda_{0} I+A\right)^{-1}\left(\frac{1}{\lambda_{0}} f+\left(1-\frac{\lambda}{\lambda_{0}}\right) u\right)
$$

Since $\left\|\lambda_{0}\left(\lambda_{0} I+A\right)^{-1}\right\| \leq 1$ and $\left|1-\frac{\lambda}{\lambda_{0}}\right|<1$ provided $\lambda<2 \lambda_{0}$, according to the fixed point theorem, (1.4) has a unique solution for all $\lambda<2 \lambda_{0}$. Repeating this procedure we obtain solution for any $\lambda>0$.

It is well known that if $X$ is a Hilbert space with inner product (.,.) then $A$ is accretive iff $\operatorname{Re}(A u, u) \geq 0$ for all $u \in D(A)$.

Definition 1.3 An operator $A$ is said to be positive if it is nonnegative and $0 \in \rho(A)$.

There are many examples of positive operators. For instance, any positive-definite selfadjoint operator acting in Hilbert space is a positive operator. If $A$ generates a $C_{0^{-}}$ semigroup of negative type then $-A$ is a positive operator. The reverse statement, however, is untrue, since there exist positive operators which are not generators of suitable semigroups. In many cases, however, condition $0 \in \rho(A)$ is not satisfied, e.g. for the Laplace operator on $L^{p}\left(\mathbb{R}^{n}\right)$ we have $0 \in \sigma(A)$. Therefore it is desirable to weaken this condition. 
Definition 1.4 An operator $A$ is called sectorial if it is nonnegative and $N(A)=\{0\}$ and $\overline{R(A)}=X$.

Obviously, any positive operator is sectorial. Examples of sectorial, but not positive operators are some differential operators on unbounded regions, like the Laplace operator or the Stokes operator on exterior domains.

For the class of sectorial operators one can define complex powers (see e.g. [64]). A sectorial operator $A$ is said to admit bounded imaginary powers if the purely imaginary powers $A^{i s}$ of $A$ are uniformly bounded for $s \in[-1,1]$. Then it can be shown that $A^{i s}$ forms a strongly continuous $C_{0}$-group of bounded linear operators. The type $\theta_{A}$ of this group defined by

$$
\theta_{A}:=\varlimsup_{|s| \rightarrow \infty}|s|^{-1} \log \left\|A^{i s}\right\|_{\mathcal{B}(X)} .
$$

is called the power angle of $A$. Then for any $\varphi_{A}>\theta_{A}$, there exists constant $M=$ $M\left(\varphi_{A}\right) \geq 1$ such that

$$
\left\|A^{i s}\right\|_{\mathcal{B}(X)} \leq M e^{\varphi_{A}|s|}, s \in \mathbb{R} .
$$

We denote $A \in \mathcal{B I P}\left(X ; M, \varphi_{A}\right)$ or $A \in \mathcal{B I P}\left(X ; \varphi_{A}\right)$. The spectral angle $\omega_{A}$ and the power angle $\theta_{A}$ of an operator satisfy the inequality $\theta_{A} \geq \omega_{A}$ (see [65]).

Let $A$ be a nonnegative operator in $X, \gamma \in(0,1), p \in(1, \infty)$. Consider the spaces

$$
D_{A}(\gamma, p):=\left\{x \in X \mid[x]_{D_{A}(\gamma, p)}<\infty\right\},
$$

where

$$
[x]_{D_{A}(\gamma, p)}:=\left\{\int_{0}^{\infty}\left(t^{\gamma}\left\|A(t I+A)^{-1} x\right\|_{X}\right)^{p} \frac{d t}{t}\right\}^{\frac{1}{p}},
$$

endowed with the norm $\|x\|_{D_{A}(\gamma, p)}:=\|x\|_{X}+[x]_{D_{A}(\gamma, p)}$. These spaces coincide up to the equivalence of norms with the real interpolation spaces $(X, D(A))_{\gamma, p}$ between $X$ and $D(A)$ ([14], Proposition 3). They are intermediate spaces between $D(A)$ and $X$ in the following sense:

$$
D(A) \hookrightarrow D_{A}(\gamma, p) \hookrightarrow D_{A}\left(\gamma^{\prime}, p\right) \hookrightarrow X, 0<\gamma^{\prime}<\gamma<1
$$

The real interpolation spaces are extensively studied; we refer e.g. to [70] for a more detailed description.

Recall that a Banach space $X$ is said to belong to the class $\mathcal{H} \mathcal{T}$ if the Hilbert transform $H$ defined by

$$
(H f)(t)=\lim _{\varepsilon \rightarrow 0+} \int_{|s| \geq \varepsilon} f(t-s) \frac{d s}{\pi s}, \quad t \in \mathbb{R}, f \in C_{0}^{\infty}(\mathbb{R} ; X),
$$

extends to a bounded linear operator on $L^{p}(\mathbb{R} ; X)$ for some $p \in(1, \infty)$. It is well known that Hilbert spaces are of class $\mathcal{H} \mathcal{T}$ and if $X$ is of class $\mathcal{H T}$ then $L^{p}(\mathbb{R} ; X)$ is of class $\mathcal{H} \mathcal{T}$ for every $p \in(1, \infty)$. Note also that any Banach space of class $\mathcal{H} \mathcal{T}$ is reflexive. 


\subsection{Fractional integration and differentiation}

Let $\alpha>0, m=\lceil\alpha\rceil$ and $I=(0, T)$ for some $T>0$. For the sake of brevity we use the following notation for $\beta \geq 0$ :

$$
g_{\beta}(t):= \begin{cases}\frac{1}{\Gamma(\beta)} t^{\beta-1}, & t>0 \\ 0, & t \leq 0\end{cases}
$$

where $\Gamma(\beta)$ is the Gamma function. Note that $g_{0}(t)=0$, because $\Gamma(0)^{-1}=0$. These functions satisfy the semigroup property

$$
g_{\alpha} * g_{\beta}=g_{\alpha+\beta}
$$

The Riemann-Liouville fractional integral of order $\alpha>0$ is defined as follows:

$$
J_{t}^{\alpha} f(t):=\left(g_{\alpha} * f\right)(t), \quad f \in L^{1}(I), t>0 .
$$

Set $J_{t}^{0} f(t):=f(t)$. Thanks to (1.8) and the associativity of the convolution we obtain that the operators of fractional integration obey the semigroup property

$$
J_{t}^{\alpha} J_{t}^{\beta}=J_{t}^{\alpha+\beta}, \quad \alpha, \beta \geq 0 .
$$

The Riemann-Liouville fractional derivative of order $\alpha$ is defined for all $f$ satisfying

$$
f \in L^{1}(I), g_{m-\alpha} * f \in W^{m, 1}(I)
$$

by

$$
D_{t}^{\alpha} f(t):=D_{t}^{m}\left(g_{m-\alpha} * f\right)(t)=D_{t}^{m} J_{t}^{m-\alpha} f(t),
$$

where $D_{t}^{m}:=\frac{d^{m}}{d t^{m}}, m \in \mathbb{N}$. As in the case of differentiation and integration of integer order, $D_{t}^{\alpha}$ is a left inverse of $J_{t}^{\alpha}$, but in general it is not a right inverse. More precisely, we have the following theorem [67]:

Theorem 1.5 Let $\alpha>0$ and $m=\lceil\alpha\rceil$. Then for any $f \in L^{1}(I)$

$$
D_{t}^{\alpha} J_{t}^{\alpha} f=f .
$$

If moreover (1.11) holds then

$$
J_{t}^{\alpha} D_{t}^{\alpha} f(t)=f(t)-\sum_{k=0}^{m-1}\left(g_{m-\alpha} * f\right)^{(k)}(0) g_{\alpha+k+1-m}(t) .
$$

In the particular case $g_{m-\alpha} * f \in W_{0}^{m, 1}(I)$, we have $J_{t}^{\alpha} D_{t}^{\alpha} f=f$.

Proof: If $f \in L^{1}(I)$, then $J_{t}^{\alpha} f$ satisfies (1.11): $J_{t}^{\alpha} f=g_{\alpha} * f \in L^{1}(I)$ and $g_{m-\alpha} *\left(J_{t}^{\alpha} f\right)=$ $g_{m-\alpha} * g_{\alpha} * f=g_{m} * f \in W_{0}^{m, 1}(I)$. So, we can apply $D_{t}^{\alpha}$ to $J_{t}^{\alpha} f$ and thanks to the semigroup property (1.10)

$$
D_{t}^{\alpha} J_{t}^{\alpha} f=D_{t}^{m} J_{t}^{m-\alpha} J_{t}^{\alpha} f=D_{t}^{m} J_{t}^{m} f=f .
$$


If $f$ satisfies (1.11), then according to (1.1),

$$
g_{m-\alpha} * f=\sum_{k=0}^{m-1} c_{k} g_{k+1}(t)+g_{m} * \varphi,
$$

where $\varphi \in L^{1}(I), c_{k}=\left(g_{m-\alpha} * f\right)^{(k)}(0)$. Therefore

$$
J_{t}^{\alpha} D_{t}^{\alpha} f=J_{t}^{\alpha} D_{t}^{m}\left(g_{m-\alpha} * f\right)=J_{t}^{\alpha} \varphi .
$$

Convolving both sides of (1.15) with $g_{\alpha}$, and applying the semigroup property (1.8), we obtain

$$
g_{m} * f=\sum_{k=0}^{m-1} c_{k} g_{\alpha+k+1}(t)+g_{\alpha+m} * \varphi .
$$

An application of $D_{t}^{m}$ to both sides gives

$$
f=\sum_{k=0}^{m-1} c_{k} g_{\alpha+k+1-m}(t)+g_{\alpha} * \varphi,
$$

which together with (1.16) implies (1.14). If $g_{m-\alpha} * f \in W_{0}^{m, 1}(I)$, that is $c_{k}=0, k=$ $0,1, \ldots, m-1$, we have $J_{t}^{\alpha} D_{t}^{\alpha} f=f$.

In particular, if $\alpha \in(0,1)$, and if $g_{1-\alpha} * f \in W^{1,1}(I)$ then (1.14) reads

$$
J_{t}^{\alpha} D_{t}^{\alpha} f(t)=f(t)-\left(g_{1-\alpha} * f\right)(0) g_{\alpha}(t) .
$$

If $f \in W^{m, 1}(I)$ (which implies (1.11)), then $D_{t}^{\alpha} f$ may be represented in he form

$$
D_{t}^{\alpha} f=\sum_{k=0}^{m-1} f^{(k)}(0) g_{k-\alpha+1}(t)+J_{t}^{m-\alpha} D_{t}^{m} f(t) .
$$

It follows from the representation (1.1) of the elements of $W^{m, 1}(I)$ and the definition of $D_{t}^{\alpha}$. In many cases it is more convenient to use the second term in the right-hand side of (1.17) as a definition of fractional derivative of order $\alpha$. The usefulness of such a definition in the mathematical analysis is demonstrated in [29]. Later, this alternative definition of fractional derivative was introduced by Caputo [10], [11], and adopted by Caputo and Mainardi [12] in the framework of the theory of linear viscoelasticity. So, the Caputo fractional derivative of order $\alpha>0$ is defined by

$$
\mathbf{D}_{t}^{\alpha} f(t):=J_{t}^{m-\alpha} D_{t}^{m} f(t) .
$$

Some simple but relevant results valid for $\alpha, \beta, t>0$ are:

$$
J_{t}^{\alpha} g_{\beta}=g_{\alpha+\beta}, \quad D_{t}^{\alpha} g_{\beta}=g_{\beta-\alpha}, \quad \beta \geq \alpha .
$$

In particular, $D_{t}^{\alpha} g_{\alpha}=0$. We also note that $D_{t}^{\alpha} 1=g_{1-\alpha}, \alpha \leq 1$, while $\mathbf{D}_{t}^{\alpha} 1=0$ for all $\alpha>0$. If instead of $f \in W^{m, 1}(I)$ we have only (1.11) and $f \in C^{m-1}(I)$, then we can use the following equivalent representation, which follows from (1.17), (1.18) and (1.19):

$$
\mathbf{D}_{t}^{\alpha} f(t)=D_{t}^{\alpha}\left(f(t)-\sum_{k=0}^{m-1} f^{(k)}(0) g_{k+1}(t)\right) .
$$


The Caputo derivative $\mathbf{D}_{t}^{\alpha}$ is again a left inverse of $J_{t}^{\alpha}$ but in general not a right inverse:

$$
\mathbf{D}_{t}^{\alpha} J_{t}^{\alpha} f=f, \quad J_{t}^{\alpha} \mathbf{D}_{t}^{\alpha} f(t)=f(t)-\sum_{k=0}^{m-1} f^{(k)}(0) g_{k+1}(t) .
$$

The first identity is valid for all $f \in L^{1}(I)$, the second for $f \in C^{m-1}(I)$, such that (1.11) is satisfied. In particular, if $\alpha \in(0,1), g_{1-\alpha} * f \in W^{1,1}(I)$ and $f \in C(I)$, then $J_{t}^{\alpha} \mathbf{D}_{t}^{\alpha} f(t)=f(t)-f(0)$.

Applying the properties of the Laplace transform and since $\widehat{g_{\alpha}}(\lambda)=\lambda^{-\alpha}$, we obtain

$$
\begin{gathered}
\widehat{D_{t}^{\alpha} f}(\lambda)=\lambda^{\alpha} \widehat{f}(\lambda)-\sum_{k=0}^{m-1}\left(g_{m-\alpha} * f\right)^{(k)}(0) \lambda^{m-1-k}, \\
\widehat{\mathbf{D}_{t}^{\alpha} f}(\lambda)=\lambda^{\alpha} \widehat{f}(\lambda)-\sum_{k=0}^{m-1} f^{(k)}(0) \lambda^{\alpha-1-k} .
\end{gathered}
$$

\subsection{Mittag-Leffler and Wright functions}

In this section we summarize some properties of two special functions which play an important role in the study of fractional differential equations.

The Mittag-Leffler function (see [32], Vol. 3, Chapter 18), is defined as follows:

$$
E_{\alpha, \beta}(z):=\sum_{n=0}^{\infty} \frac{z^{n}}{\Gamma(\alpha n+\beta)}=\frac{1}{2 \pi i} \int_{C} \frac{\mu^{\alpha-\beta} e^{\mu}}{\mu^{\alpha}-z} d \mu, \quad \alpha, \beta>0, z \in \mathbb{C},
$$

where $C$ is a contour which starts and ends at $-\infty$ and encircles the disc $|\mu| \leq|z|^{1 / \alpha}$ counter-clockwise. For short, $E_{\alpha}(z):=E_{\alpha, 1}(z)$. It is an entire function which provides a simple generalization of the exponential function: $E_{1}(z)=e^{z}$ and the cosine function: $E_{2}\left(z^{2}\right)=\cosh (z), E_{2}\left(-z^{2}\right)=\cos (z)$, and plays an important role in the theory of fractional differential equations. Similarly to the differential equation $d / d t\left(e^{\omega t}\right)=\omega e^{\omega t}$ the Mittag-Leffler function $E_{\alpha}(z)$ satisfies the more general differential relation

$$
\mathbf{D}_{t}^{\alpha} E_{\alpha}\left(\omega t^{\alpha}\right)=\omega E_{\alpha}\left(\omega t^{\alpha}\right)
$$

The most interesting properties of the Mittag-Leffler functions are associated with their Laplace integral

$$
\int_{0}^{\infty} e^{-\lambda t} t^{\beta-1} E_{\alpha, \beta}\left(\omega t^{\alpha}\right) d t=\frac{\lambda^{\alpha-\beta}}{\lambda^{\alpha}-\omega}, \quad \operatorname{Re} \lambda>\omega^{1 / \alpha}, \omega>0,
$$

and with their asymptotic expansion as $z \rightarrow \infty$. If $0<\alpha<2, \beta>0$, then

$$
E_{\alpha, \beta}(z)=\frac{1}{\alpha} z^{(1-\beta) / \alpha} \exp \left(z^{1 / \alpha}\right)+\varepsilon_{\alpha, \beta}(z), \quad|\arg z| \leq \frac{1}{2} \alpha \pi,
$$




$$
E_{\alpha, \beta}(z)=\varepsilon_{\alpha, \beta}(z), \quad|\arg (-z)|<\left(1-\frac{1}{2} \alpha\right) \pi
$$

where

$$
\varepsilon_{\alpha, \beta}(z)=-\sum_{n=1}^{N-1} \frac{z^{-n}}{\Gamma(\beta-\alpha n)}+O\left(|z|^{-N}\right), z \rightarrow \infty .
$$

Moreover, $E_{\alpha}(-x)$ is a completely monotonic function for $x \geq 0$ and $0<\alpha \leq 1$, i.e. $(-1)^{n}\left(d^{n} / d x^{n}\right) E_{\alpha}(-x) \geq 0$.

Let us consider the ordinary fractional differential equation

$$
\mathbf{D}_{t}^{\alpha} u(t)=-\omega u(t), 0<\alpha<2, \omega>0 .
$$

According to the cases $0<\alpha<1$ and $1<\alpha<2$ it can be referred to as the fractional relaxation or the fractional oscillation equation, respectively. In the former case, it must be equipped with a single initial condition, say $u(0)=u_{0}$, and in the latter with two initial conditions, say $u(0)=u_{0}$, and $u^{\prime}(0)=u_{1}$. The solution of (1.29) can be obtained by applying the Laplace transform technique which implies:

$$
\begin{aligned}
& u(t)=u_{0} E_{\alpha}\left(-\omega t^{\alpha}\right), \alpha \in(0,1), \\
& u(t)=u_{0} E_{\alpha}\left(-\omega t^{\alpha}\right)+u_{1} t E_{\alpha, 2}\left(-\omega t^{\alpha}\right), \alpha \in(1,2) .
\end{aligned}
$$

If we assume $u_{1}=0$ in order to ensure the continuous dependence of the solution of (1.29) on $\alpha$ in the transition from $\alpha=1-$ to $\alpha=1+$, we observe the following behaviour of $u(t)$. In comparison to ordinary relaxation $(\alpha=1)$ fractional relaxation for small times exhibits a much faster decay (the derivative tends to $-\infty$ in comparison to -1 ) and for large times a much slower decay (algebraic decay in comparison to exponential decay). Compared to the ordinary oscillation $(\alpha=2)$, the solution of the fractional oscillation equation does not exhibit permanent oscillations but an asymptotic algebraic decay. There are some attenuated oscillations, whose number and initial amplitude increase with $\alpha$, i.e. we observe features intermediate between relaxation and oscillation.

If we consider the analogue of (1.29) with Riemann-Liouville fractional derivative

$$
D_{t}^{\alpha} u(t)=-\omega u(t), 0<\alpha<2, \omega>0,
$$

which requires an initial condition $\left(g_{1-\alpha} * u\right)(0)=u_{0}$ when $0<\alpha<1$ and two initial conditions $\left(g_{2-\alpha} * u\right)(0)=u_{0}$ and $\left(g_{2-\alpha} * u\right)^{\prime}(0)=u_{1}$ when $1<\alpha<2$, we obtain

$$
\begin{aligned}
& u(t)=u_{0} t^{\alpha-1} E_{\alpha, \alpha}\left(-\omega t^{\alpha}\right), \alpha \in(0,1), \\
& u(t)=u_{0} t^{\alpha-2} E_{\alpha, \alpha-1}\left(-\omega t^{\alpha}\right)+u_{1} t^{\alpha-1} E_{\alpha, \alpha}\left(-\omega t^{\alpha}\right), \alpha \in(1,2) .
\end{aligned}
$$

Consider also the following function of Wright type (see [74], [54], [39], [56]):

$$
\Phi_{\gamma}(z):=\sum_{n=0}^{\infty} \frac{(-z)^{n}}{n ! \Gamma(-\gamma n+1-\gamma)}=\frac{1}{2 \pi i} \int_{\Gamma} \mu^{\gamma-1} \exp \left(\mu-z \mu^{\gamma}\right) d \mu, 0<\gamma<1,
$$


where $\Gamma$ is a contour which starts and ends at $-\infty$ and encircles the origin once counterclockwise. It is of interest because of the following relationship with the Mittag-Leffler function:

$$
E_{\gamma}(z)=\int_{0}^{\infty} \Phi_{\gamma}(t) e^{z t} d t, \quad z \in \mathbb{C}, 0<\gamma<1
$$

that is, $E_{\gamma}(-z)$ is the Laplace transform of $\Phi_{\gamma}(t)$ in the whole complex plane. Therefore, $\Phi_{\gamma}(t)$ is a probability density function:

$$
\Phi_{\gamma}(t) \geq 0, \quad t>0 ; \quad \int_{0}^{\infty} \Phi_{\gamma}(t) d t=1
$$

The identity is a particular case of (1.31). The positivity of $\Phi_{\gamma}(t)$ follows from (1.31), the complete monotonicity of $E_{\gamma}(-x)$ for $x \geq 0$ and $0<\gamma \leq 1$ and the Post-Widder inversion formula:

Lemma 1.6 Let $u(t)$ be a $X$ valued continuous function defined in $t \geq 0$ such that $u(t)=O(\exp (\gamma t))$ as $t \rightarrow \infty$ for some $\gamma$ and let $\widehat{u}(\lambda)$ be the Laplace transform of $u(t)$. Then

$$
u(t)=\lim _{n \rightarrow \infty} \frac{(-1)^{n}}{n !}\left(\frac{n}{t}\right)^{n+1}\left(\frac{\partial^{n}}{\partial \lambda^{n}} \widehat{u}\right)\left(\frac{n}{t}\right)
$$

uniformly on compacts of $t>0$.

The entire function $\Phi_{\gamma}$ has the following asymptotic expansion for $0<\gamma<1$, as $|z| \rightarrow \infty$ in the sector $|\arg z| \leq \min \{(1-\gamma) 3 \pi / 2, \pi\}-\varepsilon$ :

$$
\Phi_{\gamma}(z)=Y^{\gamma-1 / 2} e^{-Y}\left(\sum_{m=0}^{M-1} A_{m} Y^{-m}+O\left(|Y|^{-M}\right)\right)
$$

with $Y=(1-\gamma)\left(\gamma^{\gamma} z\right)^{1 /(1-\gamma)}$, where $A_{m}$ are certain real numbers ( see [74]).

\subsection{Operators of fractional differentiation in $L^{p}$ spaces}

Let $X$ be a complex Banach space. Let $\alpha>0, m=\lceil\alpha\rceil, p \in[1, \infty)$, and $I=(0, T)$ where $T>0$. In this section we study $D_{t}^{\alpha}$ as operators acting in $L^{p}(I ; X)$.

Denote the operators of fractional integration on $L^{p}(I ; X)$ by $\mathcal{J}_{\alpha}$ :

$$
D\left(\mathcal{J}_{\alpha}\right):=L^{p}(I ; X), \quad \mathcal{J}_{\alpha} u:=g_{\alpha} * u,
$$

where the integration is in the sense of Bochner. Applying the Young inequality, it follows that $\mathcal{J}_{\alpha} \in \mathcal{B}\left(L^{p}(I ; X)\right)$ :

$$
\left\|\mathcal{J}_{\alpha} u\right\|_{L^{p}(I ; X)}=\left\|g_{\alpha} * u\right\|_{L^{p}(I ; X)} \leq\left\|g_{\alpha}\right\|_{L^{1}(I)}\|u\|_{L^{p}(I ; X)}=g_{\alpha+1}(T)\|u\|_{L^{p}(I ; X)} .
$$

In fact, the following theorem can be proved just as in the scalar case: 
Theorem 1.7 Operators of fractional integration $\mathcal{J}_{\alpha}, \alpha \geq 0$, form a $C_{0}$-semigroup of bounded operators in $L^{p}(I ; X), 1 \leq p<\infty$.

Define the spaces $R^{\alpha, p}(I ; X)$ and $R_{0}^{\alpha, p}(I ; X)$ as follows. If $\alpha \notin \mathbb{N}$, set

$$
\begin{aligned}
& R^{\alpha, p}(I ; X):=\left\{u \in L^{p}(I ; X) \mid g_{m-\alpha} * u \in W^{m, p}(I ; X)\right\}, \\
& R_{0}^{\alpha, p}(I ; X):=\left\{u \in L^{p}(I ; X) \mid g_{m-\alpha} * u \in W_{0}^{m, p}(I ; X)\right\} .
\end{aligned}
$$

If $\alpha \in \mathbb{N}$ we take

$$
R^{\alpha, p}(I ; X):=W^{\alpha, p}(I ; X), \quad R_{0}^{\alpha, p}(I ; X):=W_{0}^{\alpha, p}(I ; X) .
$$

Denote the extensions of the operators of fractional differentiation in $L^{p}(I ; X)$ by $\mathcal{L}_{\alpha}$, i.e.

$$
D\left(\mathcal{L}_{\alpha}\right):=R_{0}^{\alpha, p}(I ; X), \quad \mathcal{L}_{\alpha} u:=D_{t}^{\alpha} u,
$$

where $D_{t}^{\alpha}$ is the Riemann-Liouville fractional derivative (1.12). In the next lemma we study the properties of $\mathcal{L}_{\alpha}$.

Lemma 1.8 Let $\alpha>0,1<p<\infty, X$ be a complex Banach space, and $\mathcal{L}_{\alpha}$ be the operators defined by (1.37). Then

(a) $\mathcal{L}_{\alpha}$ are closed, linear, densely defined;

(b) $\mathcal{L}_{\alpha}=\mathcal{J}_{\alpha}^{-1}$;

(c) $\mathcal{L}_{\alpha}=\mathcal{L}_{1}^{\alpha}$, the $\alpha$-th power of the operator $\mathcal{L}_{1}$;

(d) if $\alpha \in(0,2)$ then $\mathcal{L}_{\alpha}$ are positive operators with spectral angle $\omega_{\mathcal{L}_{\alpha}}=\alpha \pi / 2$;

(e) if $X$ is of class $\mathcal{H} \mathcal{T}$ and $\alpha \in(0,2)$ then $\mathcal{L}_{\alpha} \in \mathcal{B I P}\left(L^{p}(I ; X) ; \alpha(\pi / 2+\varepsilon)\right)$ for each $\varepsilon>0$

(f) if $\alpha \in(0,1]$ then $\mathcal{L}_{\alpha}$ are $m$-accretive operators.

Proof: The operator $\mathcal{J}_{\alpha}$ is injective. Indeed, if $\mathcal{J}_{\alpha} u=0$, then $\mathcal{J}_{1} u=\mathcal{J}_{1-\alpha} \mathcal{J}_{\alpha} u=0$, whence $u=0$. Therefore $\mathcal{J}_{\alpha}^{-1}$ exists. We shall prove that $\mathcal{L}_{\alpha}=\mathcal{J}_{\alpha}^{-1}$. If $u \in R\left(\mathcal{J}_{\alpha}\right)$, then $u=g_{\alpha} * v$ for some $v \in L^{p}(I ; X)$, and $g_{m-\alpha} * u=g_{m-\alpha} * g_{\alpha} * v=g_{m} * v$. Therefore, $g_{m-\alpha} * u \in W_{0}^{m, p}(I ; X)$, that is, $u \in R_{0}^{\alpha, p}=D\left(\mathcal{L}_{\alpha}\right)$. The identities $\mathcal{L}_{\alpha} \mathcal{J}_{\alpha} u=u, u \in$ $L^{p}(I ; X), \mathcal{J}_{\alpha} \mathcal{L}_{\alpha} v=v, v \in D\left(\mathcal{L}_{\alpha}\right)$, can be proven in the same way as in the scalar case, Theorem 1.5. Thus, we proved (b). The representation $\mathcal{L}_{\alpha}=\mathcal{J}_{\alpha}^{-1}$ incidentally shows that $\mathcal{L}_{\alpha}$ is a closed operator as an inverse of a bounded operator and that it is densely defined because $D\left(\mathcal{L}_{\alpha}\right)=R\left(\mathcal{J}_{\alpha}\right)$, which is dense in $L^{p}(I ; X)$. Obviously, it is also linear and (a) is proved.

Let us compute the resolvent of $\mathcal{L}_{1}$,

$$
\left(\left(s I+\mathcal{L}_{1}\right)^{-1} f\right)(t)=\int_{0}^{t} e^{-s(t-\tau)} f(\tau) d \tau, \operatorname{Re} s>0, t \in I .
$$


This representation implies that $\mathcal{L}_{1}$ is positive with spectral angle $\phi_{\mathcal{L}_{1}}=\pi / 2$. We shall prove that $\mathcal{L}_{\alpha}=\mathcal{L}_{1}^{\alpha}$. Consider first the case $\alpha \in(0,1)$. We have the following representation (see e.g. [1], eq. (4.6.9)):

$$
\mathcal{L}_{1}^{\alpha-1}=\frac{\sin \pi \alpha}{\pi} \int_{0}^{\infty} s^{\alpha-1}\left(s I+\mathcal{L}_{1}\right)^{-1} d s .
$$

Applying (1.38) and using the definition of the Gamma function and the formula

$$
\Gamma(\alpha) \Gamma(1-\alpha)=\pi / \sin \pi \alpha
$$

we obtain

$$
\mathcal{L}_{1}^{\alpha-1} f(t)=\frac{\sin \pi \alpha}{\pi} \int_{0}^{\infty} s^{\alpha-1} \int_{0}^{t} e^{-s(t-\tau)} f(\tau) d \tau d s=g_{1-\alpha} * f
$$

for $t \in I, f \in L^{p}(I ; X)$. Since $\mathcal{L}_{1}$ is an isomorphic mapping from $D\left(\mathcal{L}_{1}^{\alpha}\right)$ to $D\left(\mathcal{L}_{1}^{\alpha-1}\right)([70]$, Section 1.15.2) then $f \in D\left(\mathcal{L}_{1}^{\alpha}\right)$ is equivalent to $\mathcal{L}_{1}^{\alpha-1} f \in D\left(\mathcal{L}_{1}\right)$. This is equivalent to $f \in D\left(\mathcal{L}_{\alpha}\right)$ by $(1.39)$ and by the definition of $D\left(\mathcal{L}_{\alpha}\right)$. Therefore $D\left(\mathcal{L}_{1}^{\alpha}\right)=D\left(\mathcal{L}_{\alpha}\right)$. Applying $\mathcal{L}_{1}$ to $(1.39)$ we obtain $\mathcal{L}_{1}^{\alpha} f=\mathcal{L}_{\alpha} f$ for $f \in D\left(\mathcal{L}_{1}^{\alpha}\right)=D\left(\mathcal{L}_{\alpha}\right)$. Let now $\alpha>1$. Then from the definition of $\mathcal{L}_{\alpha}$ and the above result one has $\mathcal{L}_{\alpha}=\mathcal{L}_{m-1} \mathcal{L}_{\alpha-m+1}=\mathcal{L}_{1}^{m-1} \mathcal{L}_{1}^{\alpha-m+1}=\mathcal{L}_{1}^{\alpha}$.

The facts that $\mathcal{L}_{\alpha}, \alpha \in(0,2)$, are positive and $\phi_{\mathcal{L}_{\alpha}}=\alpha \pi / 2$ follow from the representation $\mathcal{L}_{\alpha}=\mathcal{L}_{1}^{\alpha}$. To see that $\omega_{\mathcal{L}_{\alpha}} \leq \alpha \pi / 2$ one applies [59], Proposition 4. Assume that $\omega_{\mathcal{L}_{\alpha}}<$ $\alpha \pi / 2$. Then $\phi_{\mathcal{L}_{\alpha}}>\pi(1-\alpha / 2)$. But from the representation ([67], Example 42.2)

$$
\left(\left(s I+\mathcal{L}_{\alpha}\right)^{-1} f\right)(t)=\int_{0}^{t}(t-\tau)^{\alpha-1} E_{\alpha, \alpha}\left(-s(t-\tau)^{\alpha}\right) f(\tau) d \tau
$$

and from the asymptotic expansion of the Mittag-Leffler function (1.27) it follows that if $f$ is a constant then $\left\|s\left(s I+\mathcal{L}_{\alpha}\right)^{-1} f\right\|_{L^{p}(I ; X)} \rightarrow \infty$ as $|s| \rightarrow \infty$ and $|\arg (-s)|<\alpha \pi / 2$. By this contradiction $\omega_{\mathcal{L}_{\alpha}}=\alpha \pi / 2$.

According to [28], Th.3.1, if $X$ belongs to the class $\mathcal{H} \mathcal{T}$, then the imaginary powers of $\mathcal{L}_{1}$ satisfy the estimate

$$
\left\|\mathcal{L}_{1}^{i s}\right\|_{\mathcal{B}\left(L^{p}(I ; X)\right)} \leq c\left(1+s^{2}\right) e^{\frac{\pi}{2}|s|}, s \in \mathbb{R} .
$$

Therefore, given $\varepsilon>0$, there exists $M>0$ such that

$$
\left\|\mathcal{L}_{1}^{i s}\right\|_{\mathcal{B}\left(L^{p}(I ; X)\right)} \leq M e^{\left(\frac{\pi}{2}+\varepsilon\right)|s|}, s \in \mathbb{R}
$$

which means $\mathcal{L}_{1} \in \mathcal{B I} \mathcal{P}\left(L^{p}(I ; X) ; \pi / 2+\varepsilon\right)$. Since $\mathcal{L}_{\alpha}, \alpha \in(0,2)$, are positive, their fractional powers $\mathcal{L}_{\alpha}^{z}, z \in \mathbb{C}$, are well defined and satisfy ([1], Theorem 4.6.13) $\mathcal{L}_{\alpha}^{i s}=$ $\left(\mathcal{L}_{1}^{\alpha}\right)^{i s}=\mathcal{L}_{1}^{\alpha i s}$. Therefore, by $(1.40), \mathcal{L}_{\alpha}$ has bounded imaginary powers and

$$
\left\|\mathcal{L}_{\alpha}^{i s}\right\|_{\mathcal{B}\left(L^{p}(I ; X)\right)} \leq M e^{\alpha\left(\frac{\pi}{2}+\varepsilon\right)|s|}, s \in \mathbb{R},
$$

that is $\mathcal{L}_{\alpha} \in \mathcal{B I P}\left(L^{p}(I ; X) ; \alpha(\pi / 2+\varepsilon)\right)$.

Lastly, (f) follows from [19], Theorem 3.1, because $g_{1-\alpha} \in L^{1}(I)$ is nonnegative and nonincreasing for $\alpha \in(0,1]$. 
Let us note that if $p=2$ and $X=H$ is a Hilbert space with inner product $(.,$.$) , then$ $L^{2}(I ; H)$ is again a Hilbert space with inner product

$$
(u, v)_{L^{2}(I ; H)}:=\int_{0}^{T}(u, v)(t) d t .
$$

In this case the accretivity of $\mathcal{L}_{\alpha}$ follows directly from the fact that $g_{\alpha}, \alpha \in(0,1]$, is a kernel of positive type, i.e. $\operatorname{Re}\left(g_{\alpha} * u, u\right)_{L^{2}(I ; H)} \geq 0$. This together with $\mathcal{L}_{\alpha}=\mathcal{J}_{\alpha}^{-1}$ implies

$$
\operatorname{Re}\left(\mathcal{L}_{\alpha} v, v\right)_{L^{2}(I ; H)}=\operatorname{Re}\left(\mathcal{L}_{\alpha} v, \mathcal{J}_{\alpha} \mathcal{L}_{\alpha} v\right)_{L^{2}(I ; H)} \geq 0
$$

Next we study the properties of the domains of the operators of fractional differentiation $D\left(\mathcal{L}_{\alpha}\right)$, endowed with the norm

$$
\|f\|_{R_{0}^{\alpha, p}(I ; X)}:=\left\|\mathcal{L}_{\alpha} f\right\|_{L^{p}(I ; X)} .
$$

We proved that $D\left(\mathcal{L}_{\alpha}\right)=R\left(\mathcal{J}_{\alpha}\right)$ (Lemma 1.8, (b)). So, studying the mapping properties of the operator of fractional integration we obtain some interesting properties of $D\left(\mathcal{L}_{\alpha}\right)$. The following two results can be proven in the same way as in the scalar case (see [67], Theorems 3.5 and 3.6).

Theorem 1.9 If $0<\alpha<1$ and $1<p<1 / \alpha$, then $\mathcal{J}_{\alpha} \in \mathcal{B}\left(L^{p}(I ; X), L^{q}(I ; X)\right)$, for all $q$ such that $1 \leq q<p /(1-\alpha p)$.

This theorem shows that

$$
R_{0}^{\alpha, p}(I ; X) \hookrightarrow L^{q}(I ; X), \quad 0<\alpha<1,1<p<1 / \alpha, 1 \leq q<p /(1-\alpha p) .
$$

Theorem 1.10 If $\alpha>0, p>1 / \alpha$, then $\mathcal{J}_{\alpha} \in \mathcal{B}\left(L^{p}(I ; X), C^{\alpha-1 / p}(I ; X)\right)$ if $\alpha-1 / p \notin \mathbb{N}$. If $f \in L^{p}(I ; X)$ then $\mathcal{J}_{\alpha} f(t)=o\left(t^{\alpha-1 / p}\right)$ as $t \downarrow 0$. Moreover,

$$
\mathcal{J}_{\alpha}: L^{p}(I ; X) \rightarrow h^{\alpha-1 / p}(I ; X), \quad 1 / p<\alpha<1+1 / p
$$

This theorem implies that

$$
R_{0}^{\alpha, p}(I ; X) \hookrightarrow C^{\alpha-1 / p}(I ; X), \quad \alpha>1 / p, \alpha-1 / p \notin \mathbb{N},
$$

and that all the elements of $R_{0}^{\alpha, p}$ belong to $h^{\alpha-\frac{1}{p}}(I ; X)$ if $1 / p<\alpha<1+1 / p$.

We proceed with the identification of $R_{0}^{\alpha, p}$ as domains of the fractional powers of the operator $\mathcal{L}_{1}: \mathcal{L}_{\alpha}=\mathcal{L}_{1}^{\alpha}$ (Lemma 1.8, (c)). The first straightforward consequence is that we have the embeddings

$$
R_{0}^{\beta, p}(I ; X) \hookrightarrow R_{0}^{\alpha, p}(I ; X) \hookrightarrow L^{p}(I ; X), 0<\alpha<\beta .
$$

Applying [70], Section 1.15.2, it follows that if $\alpha, \mu>0, m \in \mathbb{N}, \alpha+\mu<m$, then $\mathcal{L}_{\alpha}$ is an isomorphic mapping from $D\left(\mathcal{L}_{\alpha+\mu}\right)$ onto $D\left(\mathcal{L}_{\mu}\right)$ and from $\left(L^{p}(I ; X), D\left(\mathcal{L}_{m}\right)\right)_{\frac{\alpha+\mu}{m}, p}$ onto $\left(L^{p}(I ; X), D\left(\mathcal{L}_{m}\right)\right)_{\frac{\mu}{m}, p}$. But [70], Section 2.10.4, Theorem 1, gives

$$
\left(L^{p}(I ; X), D\left(\mathcal{L}_{m}\right)\right)_{\frac{\beta}{m}, p}=\left(L^{p}(I ; X), W_{0}^{m, p}(I ; X)\right)_{\frac{\beta}{m}, p}=W_{0}^{\beta, p}, \quad \beta-1 / p \notin \mathbb{N}_{0},
$$


where $W^{\beta, p}(I ; X)$ denotes the Sobolev space of fractional order $\beta>0[70]$ and

$$
W_{0}^{\beta, p}(I ; X):=\left\{f \in W^{\beta, p}(I ; X) \mid f^{(k)}(0)=0, k=0,1, \ldots,[\beta-1 / p]\right\}, \quad \beta-1 / p \notin \mathbb{N}_{0} .
$$

In this way we proved the following result:

Proposition 1.11 Let $\alpha, \mu>0,1<p<\infty$. Then $\mathcal{L}_{\alpha}$ is an isomorphic mapping from $R_{0}^{\alpha+\mu, p}(I ; X)$ onto $R_{0}^{\mu, p}(I ; X)$. If moreover $\mu-1 / p \notin \mathbb{N}_{0}, \alpha+\mu-1 / p \notin \mathbb{N}_{0}$, then $\mathcal{L}_{\alpha}$ is also an isomorphic mapping from $W_{0}^{\alpha+\mu, p}(I ; X)$ onto $W_{0}^{\mu, p}(I ; X)$.

Applying [70], Sections 1.15.4 and 2.10.4, we obtain for $0 \leq \alpha<\beta \leq 1,0<\gamma<1$, $\alpha(1-\gamma)+\beta \gamma-1 / p \notin \mathbb{N}_{0}$,

$$
\begin{gathered}
\left(R_{0}^{\alpha, p}(I ; X), R_{0}^{\beta, p}(I ; X)\right)_{\gamma, p}=\left(D\left(\mathcal{L}_{1}^{\alpha}\right), D\left(\mathcal{L}_{1}^{\beta}\right)\right)_{\gamma, p}=\left(L^{p}(I ; X), D\left(\mathcal{L}_{1}^{\beta}\right)\right)_{\frac{\alpha(1-\gamma)+\beta \gamma}{\beta}, p} \\
=\left(L^{p}(I ; X), D\left(\mathcal{L}_{1}\right)\right)_{\alpha(1-\gamma)+\beta \gamma, p}=\left(L^{p}(I ; X), W_{0}^{1, p}(I ; X)\right)_{\alpha(1-\gamma)+\beta \gamma, p}=W_{0}^{\alpha(1-\gamma)+\beta \gamma, p}(I ; X),
\end{gathered}
$$

and, if $\alpha \gamma-1 / p \notin \mathbb{N}_{0}$,

$$
\left(L^{p}(I ; X), R_{0}^{\alpha, p}(I ; X)\right)_{\gamma, p}=W_{0}^{\alpha \gamma, p}(I ; X) .
$$

In the case when $X$ is of class $\mathcal{H} \mathcal{T}$, we obtain a precise identification of $R_{0}^{\alpha, p}$ for $\alpha-1 / p \notin$ $\mathbb{N}_{0}$. In this case $\mathcal{L}_{1}$ has bounded imaginary powers and therefore ([70], Theorem 1.15.3)

$$
R_{0}^{\alpha, p}=D\left(\mathcal{L}_{\alpha}\right)=D\left(\mathcal{L}_{1}^{\alpha}\right)=\left[L^{p}, D\left(\mathcal{L}_{1}\right)\right]_{\alpha}, 0<\alpha<1,
$$

the complex interpolation space between $L^{p}$ and $D\left(\mathcal{L}_{1}\right) \hookrightarrow L^{p}$ of order $\alpha$. Now introduce the Bessel potential spaces defined by

$$
\begin{gathered}
H^{\alpha, p}(\mathbb{R} ; X):=\left\{\left.f\left|\exists f_{\alpha} \in L^{p}(\mathbb{R} ; X): \widetilde{f}_{\alpha}(\rho)=\right| \rho\right|^{\alpha} \widetilde{f}(\rho), \rho \in \mathbb{R}\right\},\|f\|_{H^{\alpha, p}(\mathbb{R} ; X)}:=\left\|f_{\alpha}\right\|_{L^{p}(\mathbb{R} ; X)} ; \\
H^{\alpha, p}(I ; X):=\left\{f=\left.g\right|_{I}, g \in H^{\alpha, p}(\mathbb{R} ; X)\right\}, \quad\|f\|_{H^{\alpha, p}(I ; X)}:=\inf _{g \in H^{\alpha, p}(\mathbb{R} ; X)}\|g\|_{H^{\alpha, p}(\mathbb{R} ; X)} ; \\
H_{0}^{\alpha, p}(I ; X):=\left\{f \in H^{\alpha, p}(I ; X), f^{(k)}(0)=0, k=0,1, \ldots,[\alpha-1 / p]\right\}, \quad \alpha-1 / p \notin \mathbb{N}_{0} .
\end{gathered}
$$

For $k \in \mathbb{N}$ we have $H^{k, p}(I ; X)=W^{k, p}(I ; X)$. Therefore $D\left(\mathcal{L}_{1}\right)=W_{0}^{1, p}=H_{0}^{1, p}$. If $\alpha-1 / p \notin \mathbb{N}_{0}$, then according to [70], 2.10.4, Theorem $1,\left[L^{p}, H_{0}^{1, p}\right]_{\alpha}=H_{0}^{\alpha, p}$ and therefore in the case of $\mathcal{H} \mathcal{T}$ space we obtain for $\alpha \in(0,1)$

$$
R_{0}^{\alpha, p}(I ; X)=H_{0}^{\alpha, p}(I ; X), \quad \alpha-1 / p \notin \mathbb{N}_{0} .
$$

This is also true for all $\alpha>0, \alpha-1 / p \notin \mathbb{N}_{0}$, because $\mathcal{L}_{m-1}$ is an isomorphism from $H_{0}^{\alpha, p}$ onto $H_{0}^{\alpha-m+1, p}$ and from $R_{0}^{\alpha, p}$ to $R_{0}^{\alpha-m+1, p}=H_{0}^{\alpha-m+1, p}$. The identity (1.42) has been proven in the scalar case in [67], Theorem 18.3 and Remark 18.1, applying another approach. 


\section{Chapter 2}

\section{Solution operators: generation, approximation and perturbation}

The fractional evolution equation with Caputo fractional derivative, which will be studied in the following two chapters, is formulated. The notion of solution operator plays a basic role in its study. Problems such as generation of a solution operator, conditions for its analyticity, representation in terms of the corresponding generator, perturbation, are studied.

\subsection{Solution operators}

Consider a closed linear operator $A$ densely defined in a Banach space $X$. Let $\alpha>0$ and $m=\lceil\alpha\rceil$. Given $x_{k} \in X, k=0,1, \ldots, m-1$, we investigate the following Cauchy problem for the fractional evolution equation of order $\alpha$ :

$$
\mathbf{D}_{t}^{\alpha} u(t)=A u(t), t>0 ; \quad u^{(k)}(0)=x_{k}, k=0,1, \ldots, m-1,
$$

where $\mathbf{D}_{t}^{\alpha}$ is the Caputo fractional derivative defined by (1.20).

Definition 2.1 A function $u \in C\left(\mathbb{R}_{+} ; X\right)$ is called a strong solution of (2.1) if $u \in$ $C\left(\mathbb{R}_{+} ; D(A)\right) \cap C^{m-1}\left(\mathbb{R}_{+} ; X\right), g_{m-\alpha} *\left(u-\sum_{k=0}^{m-1} u^{(k)}(0) g_{k+1}\right) \in C^{m}\left(\mathbb{R}_{+} ; X\right)$ and $(2.1)$ holds on $\mathbb{R}_{+}$.

Definition 2.2 The problem (2.1) is called well-posed if for any $x_{k} \in D(A), k=$ $0,1, \ldots, m-1$, there is a unique strong solution $u\left(t ; x_{1}, \ldots, x_{m-1}\right)$ of $(2.1)$, and $x_{k, n} \in$ $D(A), x_{k, n} \rightarrow 0$ as $n \rightarrow \infty$, imply $u\left(t ; x_{1, n}, \ldots, x_{m-1, n}\right) \rightarrow 0$ as $n \rightarrow \infty$ in $X$, uniformly on compact intervals.

Consider the following particular case of (2.1):

$$
\mathbf{D}_{t}^{\alpha} u(t)=A u(t), u(0)=x, u^{(k)}(0)=0, k=1, \ldots, m-1 .
$$


Applying (1.21), we obtain that the Cauchy problem (2.2) is well-posed iff the following Volterra integral equation

$$
u(t)=x+\int_{0}^{t} g_{\alpha}(t-s) A u(s) d s
$$

is well-posed in sense of [64], Definition 1.2. That is why we refer several times to this monograph for basic results on evolutionary integral equations. For shortness, we define solution operator of (2.2) in terms of the corresponding integral equation (2.3).

Definition 2.3 A family $\left\{S_{\alpha}(t)\right\}_{t \geq 0} \subset \mathcal{B}(X)$ is called a solution operator for (2.2) if the following conditions are satisfied:

(a) $S_{\alpha}(t)$ is strongly continuous for $t \geq 0$ and $S_{\alpha}(0)=I$;

(b) $S_{\alpha}(t) D(A) \subset D(A)$ and $A S_{\alpha}(t) x=S_{\alpha}(t) A x$ for all $x \in D(A), t \geq 0$;

(c) $S_{\alpha}(t) x$ is a solution of (2.3) for all $x \in D(A), t \geq 0$.

Following [64], problem (2.2) is well-posed iff it admits a solution operator. Moreover, if (2.2) has a solution operator $S_{\alpha}(t)$ then the corresponding problem (2.1) is uniquely solvable with solution

$$
u(t)=\sum_{k=0}^{n-1}\left(J_{t}^{k} S_{\alpha}\right)(t) x_{k},
$$

provided $x_{k} \in D(A), k=0, \ldots, m-1$. Therefore it is well-posed. For this reason we restrict ourselves to the problem (2.2).

Definition 2.4 The solution operator $S_{\alpha}(t)$ is called exponentially bounded if there are constants $M \geq 1$ and $\omega \geq 0$ such that

$$
\left\|S_{\alpha}(t)\right\| \leq M e^{\omega t}, t \geq 0
$$

An operator $A$ is said to belong to $\mathcal{C}^{\alpha}(X ; M, \omega)$, or $\mathcal{C}^{\alpha}(M, \omega)$ for short, if the problem $(2.2)$ has a solution operator $S_{\alpha}(t)$ satisfying (2.4). Denote $\mathcal{C}^{\alpha}(\omega):=\bigcup\left\{\mathcal{C}^{\alpha}(M, \omega) ; M \geq 1\right\}$, $\mathcal{C}^{\alpha}:=\bigcup\left\{\mathcal{C}^{\alpha}(\omega) ; \omega \geq 0\right\}$. In these notations $\mathcal{C}^{1}$ and $\mathcal{C}^{2}$ are the sets of all infinitesimal generators of $\boldsymbol{C}_{\mathbf{0}^{-}}$-semigroups and cosine operator families (COF), respectively. Next we give a characterization of $\mathcal{C}^{\alpha}(M, \omega)$.

Assume $A \in \mathcal{C}^{\alpha}(M, \omega)$ and let $S_{\alpha}(t)$ be the corresponding solution operator. For $\operatorname{Re} \lambda>\omega$ we define $H(\lambda) x=\int_{0}^{\infty} e^{-\lambda t} S_{\alpha}(t) x d t, \quad x \in X$. In view of $(2.4), H(\lambda) \in \mathcal{B}(X)$. Using properties b) and c) of Definition 2.3 and the identity (1.23) we obtain

$$
\lambda^{\alpha} H(\lambda) x-\lambda^{\alpha-1} x=A H(\lambda) x, x \in X ; \quad \lambda^{\alpha} H(\lambda) x-\lambda^{\alpha-1} x=H(\lambda) A x, x \in D(A) .
$$

Hence the operator $\lambda^{\alpha} I-A$ is invertible and $H(\lambda)=\lambda^{\alpha-1} R\left(\lambda^{\alpha}, A\right)$, that is

$$
\left\{\lambda^{\alpha}: \operatorname{Re} \lambda>\omega\right\} \subseteq \varrho(A)
$$


and

$$
\lambda^{\alpha-1} R\left(\lambda^{\alpha}, A\right) x=\int_{0}^{\infty} e^{-\lambda t} S_{\alpha}(t) x d t, \quad \operatorname{Re} \lambda>\omega, x \in X .
$$

Just as in the case $\alpha=1$ we have the following

Theorem 2.5 Let $\alpha>0$. Then $A \in \mathcal{C}^{\alpha}$ and the corresponding solution operator is continuous in the uniform operator topology iff $A \in \mathcal{B}(X)$.

Proof: Let $A \in \mathcal{C}^{\alpha}(M, \omega)$ and take $\lambda>\omega$. Then (2.6) implies

$$
\lambda^{\alpha-1} R\left(\lambda^{\alpha}, A\right)-\lambda^{-1} I=\int_{0}^{\infty} e^{-\lambda t}\left(S_{\alpha}(t)-I\right) d t
$$

whence

$$
\left\|\lambda^{\alpha-1} R\left(\lambda^{\alpha}, A\right)-\lambda^{-1} I\right\| \leq \int_{0}^{\infty} e^{-\lambda t} \eta(t) d t
$$

where $\eta(t)=\left\|S_{\alpha}(t)-I\right\|$ is continuous on $t \geq 0, \eta(t) \leq M e^{\omega t}+1$, and $\eta(0)=0$. Fix $\varepsilon>0$ and take $\delta>0$ such that $\eta(t) \leq \varepsilon$ if $t \in[0, \delta]$. Then

$$
\int_{0}^{\infty} e^{-\lambda t} \eta(t) d t=\int_{0}^{\delta} e^{-\lambda t} \eta(t) d t+\int_{\delta}^{\infty} e^{-\lambda t} \eta(t) d t \leq \frac{\varepsilon}{\lambda}+o\left(\frac{1}{\lambda}\right), \lambda \rightarrow \infty .
$$

Therefore, for $\lambda$ large enough we obtain $\left\|\lambda^{\alpha} R\left(\lambda^{\alpha}, A\right)-I\right\|<1$, hence $\lambda^{\alpha} R\left(\lambda^{\alpha}, A\right)$ has a bounded inverse, that is, $R\left(\lambda^{\alpha}, A\right)^{-1}=\lambda^{\alpha} I-A$ is bounded, thus $A \in \mathcal{B}(X)$.

Conversely, let $A \in \mathcal{B}(X)$ and set

$$
S_{\alpha}(t):=\sum_{n=0}^{\infty} \frac{A^{n} t^{\alpha n}}{\Gamma(\alpha n+1)} .
$$

The right-hand side of (2.7) converges in norm for every $t \geq 0$ and defines a bounded linear operator $S_{\alpha}(t)$ (note that $\Gamma(c)>0$ for $c>0$ ):

$$
\left\|S_{\alpha}(t)\right\| \leq \sum_{n=0}^{\infty} \frac{\|A\|^{n} t^{\alpha n}}{\Gamma(\alpha n+1)}=E_{\alpha}\left(\|A\| t^{\alpha}\right)
$$

Let first $\alpha \in(0,2)$. Then inequality (2.8) implies that $S_{\alpha}(t)$ is exponentially bounded. Indeed, the asymptotic expansion (1.27) and the continuity of the Mittag-Leffler function in $t \geq 0$ imply that if $\omega \geq 0$, there is a constant $C$ such that

$$
E_{\alpha}\left(\omega t^{\alpha}\right) \leq C e^{\omega^{1 / \alpha} t}, \quad t \geq 0, \alpha \in(0,2) .
$$

Therefore (2.8) and (2.9) imply

$$
\left\|S_{\alpha}(t)\right\| \leq C e^{\|A\|^{1 / \alpha} t} .
$$


If $\alpha \in[2, \infty)$, fix $k \in \mathbb{N}$ such that $k>\alpha / 2$. Then

$$
\begin{gathered}
\left\|S_{\alpha}(t)\right\| \leq \sum_{n=0}^{\infty} \frac{\|A\|^{n} t^{\alpha n}}{\Gamma(\alpha n+1)}=\sum_{n=0}^{\infty} \frac{\|A\|^{(1 / k) k n} t^{(\alpha / k) k n}}{\Gamma((\alpha / k) k n+1)} \leq \\
\sum_{n=0}^{\infty} \frac{\|A\|^{(1 / k) n} t^{(\alpha / k) n}}{\Gamma((\alpha / k) n+1)}=E_{\alpha / k}\left(\|A\|^{1 / k} t^{\alpha / k}\right) .
\end{gathered}
$$

Now we can apply (2.9) and obtain again the estimate (2.10).

Moreover, $S_{\alpha}(t)$ satisfies conditions of Definition 2.3, hence $A \in \mathcal{C}^{\alpha}\left(\|A\|^{1 / \alpha}\right)$ and $S_{\alpha}(t)$ is the corresponding solution operator. Estimating the power series yields

$$
\left\|S_{\alpha}(t)-I\right\| \leq \sum_{n=1}^{\infty} \frac{\|A\|^{n} t^{\alpha n}}{\Gamma(\alpha n+1)}=t^{\alpha}\|A\| E_{\alpha, \alpha+1}\left(\|A\| t^{\alpha}\right),
$$

therefore $\lim _{t \downarrow 0}\left\|S_{\alpha}(t)-I\right\|=0$, i.e. the solution operator is uniformly continuous.

It is well known that if $A \in \mathcal{C}^{\alpha}$ for some integer $\alpha \geq 3$ then $A$ is a bounded operator ([34], p. 99). The following theorem is a generalization of this result.

Theorem 2.6 Assume $A \in \mathcal{C}^{\alpha}$ for some $\alpha>2$. Then $A \in \mathcal{B}(X)$.

Proof: If $A \in \mathcal{C}^{\alpha}$ for some $\alpha>2$ then (2.5) implies that

$$
\Sigma_{\alpha, \omega}:=\left\{\lambda^{\alpha}: \operatorname{Re} \lambda>\omega,|\arg \lambda| \leq \pi / \alpha<\pi / 2\right\} \subseteq \rho(A) .
$$

Hence $\rho(A)$ consists of the entire complex plane with the exception of some bounded set containing the origin. If $\mu \in \mathbb{C}$ with $|\mu|$ large enough then $\mu=\lambda^{\alpha} \in \Sigma_{\alpha, \omega}$. Equations (2.4) and (2.6) imply

$$
\|\mu R(\mu, A)\| \leq \frac{M|\lambda|}{\operatorname{Re} \lambda-\omega} \leq \frac{M|\lambda|}{|\lambda| \cos (\pi / \alpha)-\omega} \rightarrow \frac{M}{\cos (\pi / \alpha)},|\mu| \rightarrow \infty .
$$

Then the following lemma ([37], Lemma 5.2) shows that $A$ is bounded.

Lemma 2.7 If $\sigma(A)$ is a bounded subset of $\mathbb{C}$ and $\|R(\mu, A)\|=O(1 /|\mu|)$ as $|\mu| \rightarrow \infty$ then $A \in \mathcal{B}(X)$.

In view of Theorem 2.6 further we will consider mainly $\alpha, 0<\alpha \leq 2$.

After easily justified differentiation under the integral sign in (2.6) we obtain

$$
\frac{\partial^{n}}{\partial \lambda^{n}}\left(\lambda^{\alpha-1} R\left(\lambda^{\alpha}\right)\right) x=(-1)^{n} \int_{0}^{\infty} t^{n} e^{-\lambda t} S_{\alpha}(t) x d t, \quad n=0,1, \ldots, x \in X, \operatorname{Re} \lambda>\omega,
$$

that together with $(2.4)$ gives

$$
\left\|\frac{\partial^{n}}{\partial \lambda^{n}}\left(\lambda^{\alpha-1} R\left(\lambda^{\alpha}, A\right)\right)\right\| \leq \frac{M n !}{(\lambda-\omega)^{n+1}}, \operatorname{Re} \lambda>\omega, n \in \mathbb{N}_{0} .
$$


It appears that this condition is also sufficient for an operator to belong to $\mathcal{C}^{\alpha}(M, \omega)$.

The following generation theorem is a particular case of a result, essentially due to Da Prato and Iannelli [25]. We use the version given in [64], Theorem 1.3, which in our case reads:

Theorem 2.8 Let $0<\alpha \leq 2$. Then $A \in \mathcal{C}^{\alpha}(M, \omega)$ iff $\left(\omega^{\alpha}, \infty\right) \subset \varrho(A)$ and

$$
\left\|\frac{\partial^{n}}{\partial \lambda^{n}}\left(\lambda^{\alpha-1} R\left(\lambda^{\alpha}, A\right)\right)\right\| \leq \frac{M n !}{(\lambda-\omega)^{n+1}}, \lambda>\omega, n \in \mathbb{N}_{0} .
$$

Let us note that inequalities (2.11) follow from their real counterparts (2.12). The proof of this fact uses Taylor series expansion for the function $\lambda^{\alpha-1} R\left(\lambda^{\alpha}, A\right)$.

The following result is an immediate consequence of the previous theorem.

Theorem 2.9 Let $0<\alpha \leq 2$. Then $A \in \mathcal{C}^{\alpha}(M, \omega)$ iff $\left(\omega^{\alpha}, \infty\right) \subset \varrho(A)$ and there is a strongly continuous operator-valued function $S(t)$ satisfying $\|S(t)\| \leq M e^{\omega t}, t \geq 0$, and such that

$$
\lambda^{\alpha-1} R\left(\lambda^{\alpha}, A\right) x=\int_{0}^{\infty} e^{-\lambda t} S(t) x d t, \quad \lambda>\omega, x \in X .
$$

If this is the case $S(t)=S_{\alpha}(t)$.

Proof: Let a function $S(t)$ with the properties mentioned above exists. After easily justified differentiation under the integral sign in (2.13) we obtain the inequalities (2.12). Applying Theorem 2.8, it results that $A \in \mathcal{C}^{\alpha}(M, \omega)$. Let $S_{\alpha}(t)$ be the corresponding solution operator. Then (2.13) holds for both $S_{\alpha}(t)$ and $S(t)$ and $S_{\alpha}(t)=S(t)$ follows from the uniqueness of the Laplace transform. The converse has already been proven before Theorem 2.5.

Next we apply Theorems 2.8 and 2.9 to study solvability and to obtain some properties of $S_{\alpha}(t)$.

Corollary 2.10 If $A \in \mathcal{C}^{1}(\omega)$ then $A \in \mathcal{C}^{\alpha}\left(\omega^{1 / \alpha}\right)$ for any $\alpha \in(0,1)$.

Proof: Let $A \in \mathcal{C}^{1}(\omega)$. Then from the Hille-Yosida theorem there exists a constant $M=M(\omega) \geq 1$ such that $R(\lambda, A)$ exists for $\lambda>\omega$ and

$$
\left\|R(\lambda, A)^{n}\right\| \leq \frac{M}{(\lambda-\omega)^{n}}, \lambda>\omega, n \in \mathbb{N}
$$

We will prove that there exists a constant $M_{1}=M_{1}(\omega, \alpha)$ such that

$$
\left\|\frac{\partial^{n}}{\partial \lambda^{n}}\left(\lambda^{\alpha-1} R\left(\lambda^{\alpha}, A\right)\right)\right\| \leq M_{1} \frac{n !}{\left(\lambda-\omega^{1 / \alpha}\right)^{n+1}}, \lambda>\omega^{1 / \alpha}, n \in \mathbb{N}_{0} .
$$


By induction in $n$ we obtain the following representation

$$
\frac{\partial^{n}}{\partial \lambda^{n}}\left(\lambda^{\alpha-1} R\left(\lambda^{\alpha}, A\right)\right)=(-1)^{n} \lambda^{-(n+1)} \sum_{k=1}^{n+1} b_{k, n+1}^{\alpha}\left(\lambda^{\alpha} R\left(\lambda^{\alpha}, A\right)\right)^{k}, \quad n \in \mathbb{N}_{0}, \quad \alpha>0,
$$

where $b_{k, n}^{\alpha}$ are given by the recurrence relations

$$
\begin{aligned}
& b_{1,1}^{\alpha}=1 \\
& b_{k, n}^{\alpha}=(n-1-k \alpha) b_{k, n-1}^{\alpha}+\alpha(k-1) b_{k-1, n-1}^{\alpha}, 1 \leq k \leq n, n=2,3, \ldots \\
& b_{k, n}^{\alpha}=0, k>n, n=1,2, \ldots
\end{aligned}
$$

In the considered case $\alpha \in(0,1)$ we obtain from (2.17) that $b_{k, n}^{\alpha}>0$. Then from (2.16) and (2.14) we have for $\lambda>\omega^{1 / \alpha}$

$$
\begin{aligned}
\left\|\frac{\partial^{n}}{\partial \lambda^{n}}\left(\lambda^{\alpha-1} R\left(\lambda^{\alpha}, A\right)\right)\right\| & \leq \sum_{k=1}^{n+1} b_{k, n+1}^{\alpha} \lambda^{k \alpha-n-1}\left\|R\left(\lambda^{\alpha}, A\right)^{k}\right\| \\
& \leq M \sum_{k=1}^{n+1} b_{k, n+1}^{\alpha} \frac{\lambda^{k \alpha-n-1}}{\left(\lambda^{\alpha}-\omega\right)^{k}}=M(-1)^{n} \frac{\partial^{n}}{\partial \lambda^{n}}\left(\frac{\lambda^{\alpha-1}}{\lambda^{\alpha}-\omega}\right),
\end{aligned}
$$

where the last equality follows as a particular case of (2.16) with $A=\omega$.

Differentiating under the integral sign (1.26) with $\beta=1$, we have

$$
(-1)^{n} \frac{\partial^{n}}{\partial \lambda^{n}}\left(\frac{\lambda^{\alpha-1}}{\lambda^{\alpha}-\omega}\right)=\int_{0}^{\infty} t^{n} e^{-\lambda t} E_{\alpha}\left(\omega t^{\alpha}\right) d t
$$

Applying (2.9), we obtain

$$
(-1)^{n} \frac{\partial^{n}}{\partial \lambda^{n}}\left(\frac{\lambda^{\alpha-1}}{\lambda^{\alpha}-\omega}\right) \leq C \frac{n !}{\left(\lambda-\omega^{1 / \alpha}\right)^{n+1}}, \quad n \in \mathbb{N}_{0}
$$

This inequality together with (2.18) implies (2.15). Therefore $A \in \mathcal{C}^{\alpha}\left(\omega^{1 / \alpha}\right)$.

To obtain a representation of the solution operator $S_{\alpha}(t)$ in terms of the resolvent operator of $A$ we apply the Post-Widder inversion formula ( Lemma 1.6 ). It together with (2.16), which holds for any $\alpha>0$, gives:

Proposition 2.11 Let $\alpha>0$ and $A \in \mathcal{C}^{\alpha}$. Then the corresponding solution operator is given by:

$$
S_{\alpha}(t) x=\lim _{n \rightarrow \infty} \frac{1}{n !} \sum_{k=1}^{n+1} b_{k, n+1}^{\alpha}\left(I-(t / n)^{\alpha} A\right)^{-k} x,
$$

where $b_{k, n}^{\alpha}$ are the constants in (2.17). The convergence is uniform on bounded subsets of $t \geq 0$ for any fixed $x \in X$. 
This is a generalization of the exponential representation for $C_{0}$-semigroups

$$
S_{1}(t) x=\lim _{n \rightarrow \infty}\left(I-\frac{t}{n} A\right)^{-n} x, x \in X,
$$

uniformly on bounded subintervals of $t \geq 0$.

Next we express the operator $A$ in terms of the corresponding solution operator $S_{\alpha}(t)$ of the Cauchy problem (2.2).

Proposition 2.12 If $S_{\alpha}(t)$ is the solution operator of (2.2) then

$$
A x=\Gamma(\alpha+1) \lim _{t \downarrow 0} \frac{S_{\alpha}(t) x-x}{t^{\alpha}}
$$

for those $x \in X$ for which this limit exists.

Proof: For any function $v(.) \in C\left(\mathbb{R}_{+} ; X\right)$ we have:

$$
\lim _{t \downarrow 0} v(t)=\lim _{t \downarrow 0} \frac{J_{t}^{\alpha} v(t)}{g_{\alpha+1}(t)} .
$$

Taking $v(t)=\left(\mathbf{D}_{t}^{\alpha} S_{\alpha}\right)(t) x$ and using (2.2) and (1.21), we obtain (2.22).

Incidentally, $(2.22)$ shows that $S_{\alpha}(t) x$ with $\alpha \in(0,1)$ is not differentiable at $t=0^{+}$.

\section{$2.2 \quad$ Analytic solution operators}

Definition 2.13 A solution operator $S_{\alpha}(t)$ of (2.2) is called analytic if $S_{\alpha}(t)$ admits an analytic extension to a sector $\Sigma_{\theta_{0}}$ for some $\theta_{0} \in(0, \pi / 2]$. An analytic solution operator is said to be of analyticity type $\left(\theta_{0}, \omega_{0}\right)$ if for each $\theta<\theta_{0}$ and $\omega>\omega_{0}$ there is $M=M(\theta, \omega)$ such that

$$
\left\|S_{\alpha}(t)\right\| \leq M e^{\omega \operatorname{Re} t}, t \in \Sigma_{\theta} .
$$

The set of all operators $A \in \mathcal{C}^{\alpha}$, generating analytic solution operators $S_{\alpha}(t)$ of type $\left(\theta_{0}, \omega_{0}\right)$ is denoted by $\mathcal{A}^{\alpha}\left(\theta_{0}, \omega_{0}\right)$. In addition, denote $\mathcal{A}^{\alpha}\left(\theta_{0}\right):=\bigcup\left\{\mathcal{A}^{\alpha}\left(\theta_{0}, \omega_{0}\right) ; \omega_{0} \in \mathbb{R}_{+}\right\}$, $\mathcal{A}^{\alpha}:=\bigcup\left\{\mathcal{A}^{\alpha}\left(\theta_{0}\right) ; \theta_{0} \in(0, \pi / 2]\right\}$. For $\alpha=1$ we obtain the set of all generators of analytic semigroups.

Analytic solution operators have been introduced by Da Prato and Iannelli [24], who also proved a weaker version of the generation theorem [64], Theorem 2.1. The following characterization of $\mathcal{A}^{\alpha}\left(\theta_{0}, \omega_{0}\right)$ is a particular case of this theorem.

Theorem 2.14 Let $\alpha \in(0,2)$. A linear closed densely defined operator $A$ belongs to $\mathcal{A}^{\alpha}\left(\theta_{0}, \omega_{0}\right)$ iff $\lambda^{\alpha} \in \rho(A)$ for each $\lambda \in \Sigma_{\theta_{0}+\pi / 2}\left(\omega_{0}\right)$, and for any $\omega>\omega_{0}, \theta<\theta_{0}$, there is a constant $C=C(\theta, \omega)$ such that

$$
\left\|\lambda^{\alpha-1} R\left(\lambda^{\alpha}, A\right)\right\| \leq \frac{C}{|\lambda-\omega|}, \lambda \in \Sigma_{\theta+\pi / 2}(\omega)
$$


Proof: Here we present only the proof of the sufficiency part. For the complete proof we refer to [64], Theorem 2.1. Let $t \in \Sigma_{\theta}$ for some $\theta<\theta_{0}$ and $\delta \in\left(\theta, \theta_{0}\right), \omega>\omega_{0}, \varrho>0$. Set

$$
S_{\alpha}(t):=\frac{1}{2 \pi i} \int_{\Gamma} e^{\lambda t} \lambda^{\alpha-1} R\left(\lambda^{\alpha}, A\right) d \lambda
$$

where

$\Gamma:=\left\{\omega+r e^{-i(\pi / 2+\delta)} ; \varrho \leq r<\infty\right\} \cup\left\{\omega+\varrho e^{i \varphi} ;|\varphi| \leq \pi / 2+\delta\right\} \cup\left\{\omega+r e^{i(\pi / 2+\delta)} ; \varrho \leq r<\infty\right\}$

is oriented counterclockwise. Let $\varrho=1 /|t|$ and $a=\sin (\delta-\theta)$. Then from (2.25) and (2.24) it follows

$$
\left\|S_{\alpha}(t)\right\| \leq \frac{C}{2 \pi} \int_{\Gamma} e^{\operatorname{Re}(\lambda t)} \frac{|d \lambda|}{|\lambda-\omega|} \leq \frac{C e^{\omega \operatorname{Re} t}}{\pi}\left(\int_{1}^{\infty} e^{-a r} \frac{d r}{r}+\int_{0}^{\pi} e^{\cos \varphi} d \varphi\right) \leq M e^{\omega \operatorname{Re} t} .
$$

This estimate shows that the integral in (2.25) is absolutely convergent for $t \in \Sigma_{\theta}$, hence $S_{\alpha}(t)$ is analytic in this region and estimate (2.23) holds.

Now fix $\lambda>\omega$, and take $\varrho<\lambda$. Then for the Laplace transform of $S_{\alpha}(t)$, defined by (2.25), we obtain

$$
\begin{gathered}
\int_{0}^{\infty} e^{-\lambda t} S_{\alpha}(t) d t=\frac{1}{2 \pi i} \int_{\Gamma} \mu^{\alpha-1} R\left(\mu^{\alpha}, A\right) \int_{0}^{\infty} e^{-(\lambda-\mu) t} d t d \mu= \\
\frac{-1}{2 \pi i} \int_{\Gamma} \frac{\mu^{\alpha-1} R\left(\mu^{\alpha}, A\right) x}{\mu-\lambda} d \mu=\lambda^{\alpha-1} R\left(\lambda^{\alpha}, A\right),
\end{gathered}
$$

where Fubini's theorem and Cauchy's integral formula are used. So, we have proved that the conditions of Theorem 2.9 are fulfilled. Therefore $A \in \mathcal{C}^{\alpha}$ and the corresponding solution operator is $S_{\alpha}(t)$.

Next we prove an important property of the analytic solution operators (see also [64], Theorem 2.2 (ii)).

Proposition 2.15 Let $\alpha \in(0,2)$ and assume $A \in \mathcal{A}^{\alpha}\left(\theta_{0}, \omega_{0}\right)$. Then for any $x \in X$ and $t>0$ we have $S_{\alpha}(t) x \in D(A)$ and

$$
\left\|A S_{\alpha}(t)\right\| \leq C e^{\omega t}\left(1+t^{-\alpha}\right), \quad t>0, \omega>\omega_{0} .
$$

Proof: If $t>0$, applying $A$ to both sides of (2.25) and using the identity $A R(\lambda)=$ $\lambda R(\lambda)-I$, we have

$$
A S_{\alpha}(t)=\frac{1}{2 \pi i} \int_{\Gamma} e^{\lambda t} \lambda^{\alpha-1} A R\left(\lambda^{\alpha}\right) d \lambda=\frac{1}{2 \pi i} \int_{\Gamma} e^{\lambda t} \lambda^{2 \alpha-1} R\left(\lambda^{\alpha}\right) d \lambda-a(t),
$$

where $a(t)=g_{1-\alpha}(t)$ if $\alpha \in(0,1)$ and $a(t)=0$ if $\alpha \in(1,2)$. There exists a constant $c=c(\omega, \theta)$ such that

$$
\left|\lambda^{\alpha}\right| \leq c\left(|\lambda-\omega|^{\alpha}+1\right), \quad \lambda \in \Sigma_{\pi / 2+\theta}(\omega)
$$


Applying this inequality and (2.24) to (2.28) we obtain

$$
\begin{aligned}
\left\|A S_{\alpha}(t)\right\| & \leq C_{1} \int_{\Gamma} e^{\operatorname{Re} \lambda t}\left(|\lambda-\omega|^{\alpha}+1\right) \frac{|d \lambda|}{|\lambda-\omega|}+a(t) \\
& \leq C_{1} e^{\omega t}\left(\int_{\rho}^{\infty} e^{-a r t}\left(r^{\alpha}+1\right) \frac{d r}{r}+\int_{0}^{\pi} e^{\rho t \cos \varphi}\left(\rho^{\alpha}+1\right) d \varphi\right)+a(t)
\end{aligned}
$$

and (2.27) follows taking $\varrho=1 / t$.

As a byproduct of this proposition we obtain that for any $x \in X, S_{\alpha}(t) x$ is a strong solution of (2.2) in $t>0$. In contrast to analytic semigroups, we can not proceed beyond Proposition 2.15. It is not true in general that $A^{2} S_{\alpha}(t)$ is a bounded operator for $t>0$, see [64], Theorem 2.2 (iii).

We formulate two immediate corollaries of Theorem 2.14 for $\omega_{0}=0$.

Corollary 2.16 Let $\alpha \in(0,2)$. Then $A \in \mathcal{A}^{\alpha}\left(\theta_{0}, 0\right)$ iff $\rho(A) \supset \Sigma_{\alpha\left(\pi / 2+\theta_{0}\right)}$ and for all $\theta<\theta_{0}$

$$
\|R(\lambda, A)\| \leq C|\lambda|, \quad \lambda \in \Sigma_{\alpha(\pi / 2+\theta)}
$$

Note that inequality (2.29) is satisfied iff $-A$ is a positive operator with spectral angle $\omega_{-A} \leq \pi-\alpha(\pi / 2+\theta)$.

Corollary 2.17 If $\varrho(A) \supset\{\lambda: \operatorname{Re} \lambda>0\}$ and for some constant $C$

$$
\|R(\lambda, A)\| \leq C / \operatorname{Re} \lambda, \quad \operatorname{Re} \lambda>0 .
$$

then for any $\alpha \in(0,1), A \in \mathcal{A}^{\alpha}(\min \{(1 / \alpha-1) \pi / 2, \pi / 2\}, 0)$.

Proof: Fix $\alpha \in(0,1)$ and $\theta_{0}=(1 / \alpha-1) \pi / 2$. Then $\alpha\left(\pi / 2+\theta_{0}\right)<\pi / 2$ and so, $\Sigma_{\alpha\left(\pi / 2+\theta_{0}\right)} \subset$ $\varrho(A)$. Taking $\beta$, such that $\alpha\left(\pi / 2+\theta_{0}\right)<\beta<\pi / 2$, we obtain

$$
\|R(\lambda, A)\| \leq \frac{C}{\operatorname{Re} \lambda}=\frac{C}{|\lambda| \cos \varphi}<\frac{C}{|\lambda| \cos \beta}, \quad \lambda \in \Sigma_{\alpha\left(\pi / 2+\theta_{0}\right)},
$$

where $\varphi=\arg \lambda$ and so, (2.29) holds.

It is instructive to consider some simple examples.

Example 2.18 Consider the fractional diffusion-wave equation for $u=u(x, t)$

$$
\mathbf{D}_{t}^{\alpha} u=k^{2} u_{x x},-\infty<x<\infty, t>0,0<\alpha<2, k \in \mathbb{R}
$$

with conditions $u(\mp \infty, t)=0, u(x, 0)=f(x), u_{t}(x, 0)=0$ (the last one only when $1<\alpha<2)$. Let $X=L^{p}(\mathbb{R}), A=k^{2} D_{x}^{2}$ with $D(A)=W^{2, p}(\mathbb{R})$.

The fractional diffusion equation $(\alpha \in(0,1))$ has been explicitly introduced in physics by Nigmatullin [61] to describe diffusion in special types of porous media, which exhibit 
a fractal geometry. Mainardi [53] has shown that fractional wave equation $(\alpha \in(1,2))$ governs the propagation of mechanical diffusive waves in viscoelastic media which exhibit a simple power-law creep. It is proven in [35] that $(2.31)$ with $\alpha \in(1,2)$ interpolates between diffusion and wave equations.

It is well known that $A$ generates an analytic semigroup of angle $\pi / 2$, that is,

$$
\|R(\lambda, A)\| \leq M /|\lambda|, \lambda \in \Sigma_{\pi-\varepsilon} .
$$

Then Corollary 2.16 implies $A \in \mathcal{A}^{\alpha}\left(\min \left\{\frac{2-\alpha}{2 \alpha} \pi, \frac{1}{2} \pi\right\}, 0\right), \alpha \in(0,2)$. Problem (2.31) is studied further in Chapter 3, Example 3.6. Similar results hold for a more general fractional diffusion-wave problems in $\mathbb{R}^{N}$ with $A=\Delta$, the Laplace operator in $L^{p}(\Omega)$, where $\Omega \subset \mathbb{R}^{N}$ is open with, say, Dirichlet boundary conditions.

Example 2.19 Let $\alpha \in(0,1), \theta \in[0, \pi)$, and consider the problem

$$
\mathbf{D}_{t}^{\alpha} u=-e^{i \theta} u_{x}, 0<x<1, t>0 ; u(0, t)=0, u(x, 0)=f(x) .
$$

Set $X=L^{p}(0,1), A_{\theta}=-e^{i \theta} D_{x}^{1}$ with $D\left(A_{\theta}\right)=\left\{f \in W^{1, p}(0,1), f(0)=0\right\}$. It is well known that $A_{0}$ generates a contraction $C_{0}$-semigroup:

$$
\left\|R\left(\lambda, A_{0}\right)\right\| \leq 1 / \operatorname{Re} \lambda, \quad \operatorname{Re} \lambda>0,
$$

and using Corollary 2.17 it follows that $A_{0} \in \mathcal{A}^{\alpha}(\min \{(1 / \alpha-1) \pi / 2, \pi / 2\}, 0)$. For general $\theta \in(0, \pi)$ we have by $(2.32)$

$$
\left\|R\left(\lambda, A_{\theta}\right)\right\|=\left\|R\left(\lambda, e^{i \theta} A_{0}\right)\right\|=\left\|R\left(\lambda e^{-i \theta}, A_{0}\right)\right\| \leq \frac{1}{|\lambda| \cos (\varphi-\theta)}, \lambda \in \Sigma_{\pi / 2-\theta},
$$

where $\varphi=\arg \lambda$. Therefore $\Sigma_{\pi / 2-\theta} \in \rho\left(A_{\theta}\right)$ and

$$
\left\|R\left(\lambda, A_{\theta}\right)\right\| \leq \frac{M(\varepsilon)}{|\lambda|}, \quad \lambda \in \Sigma_{\pi / 2-\theta-\varepsilon},
$$

whence $A_{\theta} \in \mathcal{A}^{\alpha}\left(\min \left\{\frac{\pi / 2-\theta}{\alpha}-\frac{\pi}{2}, \frac{\pi}{2}\right\}, 0\right)$ if $|\theta|<(1-\alpha) \pi / 2$. Note that $A_{\theta} \in \mathcal{C}^{1}$ only if $\theta=0$.

This property can also be derived from the following explicit representation of the solution obtained by applying the Laplace transform method:

$$
u(x, t)=e^{-i \theta} t^{-\alpha} \int_{0}^{x} \Phi_{\alpha}\left(s e^{-i \theta} t^{-\alpha}\right) f(x-s) d s
$$

for $|\theta|<(1-\alpha) \pi / 2$, where $\Phi_{\alpha}$ is defined by (1.30).

Example 2.20 Let $\alpha \in(0,2), \theta \in[0, \pi)$, and consider the problem

$$
\mathbf{D}_{t}^{\alpha} u=e^{i \theta} u_{x x}, \quad 0<x<1, t>0 ;
$$


with initial and boundary conditions $u(0, t)=u(1, t)=0, u(x, 0)=f(x), u_{t}(x, 0)=0$ (the second only if $\alpha \in(1,2)$ ).

Take $X=L^{2}(0,1), B_{\theta}=e^{i \theta} D_{x}^{2}, D\left(B_{\theta}\right)=\left\{f \in W^{2,2}(0,1), f(0)=f(1)=0\right\}$. Since $B_{\theta}$ has eigenvalues $z_{n}=-e^{i \theta} n^{2} \pi^{2}$ and eigenfunctions $\sin n \pi x$, then if $f(x)=\sum_{n=1}^{\infty} c_{n} \sin n \pi x$ the solution is represented by the series

$$
u(x, t)=\sum_{n=1}^{\infty} c_{n} \sin n \pi x E_{\alpha}\left(z_{n} t^{\alpha}\right) .
$$

Now it is clear from the asymptotic expansion of the Mittag-Leffler function (1.27) and (1.28) that $B_{\theta} \in \mathcal{C}^{\alpha}(0)$ iff $|\theta| \leq(1-\alpha / 2) \pi$. Moreover, if $|\theta|<(1-\alpha / 2) \pi$, then $B_{\theta} \in \mathcal{A}^{\alpha}(0)$. So, $B_{\theta}$ with $\pi / 2<|\theta|<(1-\alpha / 2) \pi$, is another example of an operator generating analytic solution of (2.2) but not generating $C_{0}$-semigroup, because no right half-plane is free of spectra of $B_{\theta}$.

\subsection{Approximation}

In this section we prove directly a representation of type (2.20) and show its importance for the numerical approximation of the solution operator $S_{\alpha}(t)$, especially for implicit approximation schemes.

Let us first consider the case $\alpha=1$. Usually, to find an approximation of the value of the solution of the problem

$$
u^{\prime}(t)=A u(t), u(0)=x,
$$

at a fixed time $t>0$, we divide the interval $[0, t]$ into $n$ equal parts and approximate the derivative by a difference. If we take the two-point backward difference, we obtain the following implicit difference scheme

$$
\frac{1}{h}\left[u_{n}(j h)-u_{n}((j-1) h)\right]=A u_{n}(j h), j=1, \ldots, n, \quad u_{n}(0)=x,
$$

with $h=t / n$. The equations (2.34) can be solved explicitly and their solution $u_{n}(t)$, given by $u_{n}(t)=\left(I-\frac{t}{n} A\right)^{-n} x$ for $n$ sufficiently large, is an approximation of the solution $u(t)$ of (2.33). Then the exponential formula (2.21) implies that $u_{n}(t) \rightarrow u(t)$, as $n \rightarrow \infty$, uniformly on bounded subsets of $t \geq 0$. So, the solution of the difference scheme (2.34) converges to the solution of the differential equation (2.33).

We generalize these results to the case of the fractional order problem (2.2). Consider the numerical method for solving fractional differential equations using the following approximation of $D_{t}^{\alpha}$ by the backward fractional difference (see [63], Chapters 7 and 8):

$$
D_{\tau}^{\alpha} f(\tau) \approx h^{-\alpha} \sum_{i=0}^{\lfloor\tau / h\rfloor}(-1)^{i}\left(\begin{array}{c}
\alpha \\
i
\end{array}\right) f(\tau-i h),
$$

where

$$
\left(\begin{array}{c}
\alpha \\
i
\end{array}\right)=\frac{\alpha(\alpha-1) \ldots(\alpha-i+1)}{i !}, i \in \mathbb{N}
$$


Applying (1.20) to the solution $u(t)$ of $(2.2)$ we obtain $\mathbf{D}_{t}^{\alpha} u(t)=D_{t}^{\alpha}(u(t)-x)$. Then using (2.35) to approximate the equation in (2.2) and forward differences

$$
u^{(k)}(0) \approx h^{-k} \sum_{i=0}^{k}(-1)^{i}\left(\begin{array}{c}
k \\
i
\end{array}\right) u((k-i) h), k=0,1, \ldots, m-1,
$$

to approximate the initial conditions in $(2.2)$, we obtain the following difference scheme

$$
\begin{gathered}
h^{-\alpha} \sum_{i=0}^{j}(-1)^{i}\left(\begin{array}{c}
\alpha \\
i
\end{array}\right)\left(u_{n}((j-i) h)-x\right)=A u_{n}(j h), j=m, m+1, \ldots \\
u_{n}(j h)=x, j=0,1, \ldots, m-1,
\end{gathered}
$$

where $h=t / n$. It can be solved explicitly and the result is presented in the next

Theorem 2.21 Let $\alpha>0, A \in \mathcal{C}^{\alpha}(M, \omega)$ and $u_{n}(t)$ be the approximation defined by (2.36), (2.37) to the solution $u(t)$ of (2.2). Then

$$
u_{n}(t)=\frac{1}{(n-m) !} \sum_{k=1}^{n-m+1} b_{k, n-m+1}^{\alpha}\left(I-(t / n)^{\alpha} A\right)^{-k} x, x \in X,
$$

where $b_{k, n}^{\alpha}$ are given by the reccurence relations (2.17) and $u_{n}(t)$ converges to $u(t)$ as $n \rightarrow \infty$ uniformly on bounded subsets of $t \geq 0$.

Proof: The case $t=0$ is trivial, applying $\sum_{k=1}^{n+1} b_{k, n+1}^{\alpha}=n$ !, which can be obtained from (2.16) with $A \equiv 0$. Consider $t>0$. Then, using (2.37) and the identity

$$
\sum_{i=0}^{j}(-1)^{i}\left(\begin{array}{c}
\alpha \\
i
\end{array}\right)=\left(\begin{array}{c}
j-\alpha \\
j
\end{array}\right)
$$

we rearrange (2.36), obtaining (here we let $\sum_{i=1}^{0}=0$ ):

$$
\left(I-h^{\alpha} A\right) u_{n}((m+j) h)=\sum_{i=1}^{j}(-1)^{i+1}\left(\begin{array}{c}
\alpha \\
i
\end{array}\right) u_{n}((m+j-i) h)+\left(\begin{array}{c}
j-\alpha \\
j
\end{array}\right) x, j=0,1, \ldots
$$

If we choose $n$ such that $n / t>\omega$ and apply $(2.6)$ then $\left(I-h^{\alpha} A\right)^{-1}=h^{-\alpha} R\left(h^{-\alpha}, A\right)$ exists and we obtain from $(2.39)$

$$
u_{n}((m+j) h)=h^{-\alpha} R\left(h^{-\alpha}, A\right)\left[\sum_{i=1}^{j}(-1)^{i+1}\left(\begin{array}{c}
\alpha \\
i
\end{array}\right) u_{n}((m+j-i) h)+\left(\begin{array}{c}
j-\alpha \\
j
\end{array}\right) x\right] .
$$

Denote for shortness $F(\lambda):=\lambda^{\alpha-1} R\left(\lambda^{\alpha}, A\right)$. Next we shall prove by induction on $j$ that $u_{n}((m+j) h)=v_{n}(j, h), j=0,1, \ldots$, where

$$
v_{n}(j, h):=\left[\frac{(-1)^{j} \lambda^{j+1}}{j !} F^{(j)}(\lambda) x\right]_{\lambda=1 / h}, j=0,1, \ldots
$$


For $j=0$ this is trivial. Suppose that $u_{n}((m+l) h)=v_{n}(l, h)$ is true for all $l \leq j-1$. By (2.40) and the induction hypothesis it follows

$$
u_{n}((m+j) h)=\left[\lambda F(\lambda)\left((-1)^{j+1} \sum_{i=1}^{j}\left(\begin{array}{c}
\alpha \\
i
\end{array}\right) \frac{\lambda^{j+1-i}}{(j-i) !} F^{(j-i)}(\lambda) x+\left(\begin{array}{c}
j-\alpha \\
j
\end{array}\right) x\right)\right]_{\lambda=1 / h} .
$$

Further, we use the identity

$$
A F^{(j)}(\lambda) x=\left(\lambda^{\alpha} F(\lambda)\right)^{(j)} x+(-1)^{j+1} j ! \lambda^{-j+\alpha-1}\left(\begin{array}{c}
j-\alpha \\
j
\end{array}\right) x, j \in \mathbb{N}_{0},
$$

which can be easily proven by induction. Note that $A F^{(j)}(\lambda)$ is a bounded operator by (2.16) and the fact that $A R\left(\lambda^{\alpha}, A\right)$ is bounded. So, it is sufficient to prove (2.43) for $x \in D(A)$. Applying the operator $-R\left(\lambda^{\alpha}, A\right)$ to both sides of (2.43) and using that $A R\left(\lambda^{\alpha}, A\right) x=R\left(\lambda^{\alpha}, A\right) A x=\lambda F(\lambda) x-x, x \in D(A)$, and $R\left(\lambda^{\alpha}, A\right)=\lambda^{1-\alpha} F(\lambda)$, we obtain

$$
F^{(j)}(\lambda) x=F(\lambda)\left[-\lambda^{1-\alpha}\left(\lambda^{\alpha} F(\lambda)\right)^{(j)} x+\lambda F^{(j)}(\lambda) x+(-1)^{j} j ! \lambda^{-j}\left(\begin{array}{c}
j-\alpha \\
j
\end{array}\right) x\right] .
$$

With the aid of the Leibniz rule

$$
\left(\lambda^{\alpha} F(\lambda)\right)^{(j)}=\sum_{i=0}^{j}\left(\begin{array}{l}
j \\
i
\end{array}\right) \alpha(\alpha-1) \ldots(\alpha-i+1) \lambda^{\alpha-i} F^{(j-i)}(\lambda)
$$

(2.44) can be written in the form

$$
F^{(j)}(\lambda) x=\frac{(-1)^{j} j !}{\lambda^{j+1}} \lambda F(\lambda)\left[(-1)^{j+1} \sum_{i=1}^{j}\left(\begin{array}{c}
\alpha \\
i
\end{array}\right) \frac{\lambda^{j+1-i}}{(j-i) !} F^{(j-i)}(\lambda) x+\left(\begin{array}{c}
j-\alpha \\
j
\end{array}\right) x\right] .
$$

Now (2.42) and (2.45) imply $u_{n}((m+j) h)=v_{n}(j, h)$. Taking $j=n-m$ we obtain

$$
u_{n}(t)=\left[\frac{(-1)^{n-m} \lambda^{n-m+1}}{(n-m) !} F^{(n-m)}(\lambda) x\right]_{\lambda=n / t},
$$

and this representation together with (2.16) implies (2.38).

Differentiating (2.6) $n-m$ times with respect to $\lambda$ and inserting the result into (2.46) we find

$$
u_{n}(t)=\left[\frac{\lambda^{n-m+1}}{(n-m) !} \int_{0}^{\infty} s^{n-m} e^{-\lambda s} S_{\alpha}(s) x d s\right]_{\lambda=n / t} .
$$

Noting that $\lambda^{n-m+1} \int_{0}^{\infty} s^{n-m} e^{-\lambda s} d s=(n-m)$ !, it follows

$$
u(t)-u_{n}(t)=\left[\frac{\lambda^{n-m+1}}{(n-m) !} \int_{0}^{\infty} s^{n-m} e^{-\lambda s}\left(S_{\alpha}(t) x-S_{\alpha}(s) x\right) d s\right]_{\lambda=n / t},
$$

and after the change of variables $s=t \sigma$ we obtain

$$
u(t)-u_{n}(t)=\frac{n^{n-m+1}}{(n-m) !} \int_{0}^{\infty} \sigma^{n-m} e^{-n \sigma}\left(S_{\alpha}(t) x-S_{\alpha}(t \sigma) x\right) d \sigma .
$$

It remains to apply the following lemma and the proof of Theorem 2.21 is completed. 
Lemma 2.22 Let $f(\cdot):[0, \infty) \rightarrow X$ be a continuous function such that $\|f(t)\| \leq$ $M e^{\omega t}, t \geq 0$, for some $M \geq 1$ and $\omega \geq 0$. Then for any integer $k \geq 0$ we have

$$
\lim _{n \rightarrow \infty} \frac{n^{n-k+1}}{(n-k) !} \int_{0}^{\infty} \sigma^{n-k} e^{-n \sigma}[f(t)-f(t \sigma)] d \sigma=0
$$

uniformly on bounded subsets of $t \geq 0$.

This lemma can be proven by a method similar to that in [62], p.34, so we omit the proof. The representation (2.20) is (2.38) with $m=0$. In fact, (2.49) together with Lemma 2.22 shows that no matter what integer $m \geq 0$ we take in the representation (2.46), resp. (2.47), of $u_{n}(t)$ we always have $u_{n}(t) \rightarrow u(t)$ as $n \rightarrow \infty$.

Next we estimate the rate of convergence of representation (2.38) when $\alpha \geq 1$.

Theorem 2.23 Let $\alpha \geq 1, A \in \mathcal{C}^{\alpha}(M, \omega)$ and $x \in D(A)$. If $u_{n}(t)$ is the approximation defined by (2.36) and (2.37) to the solution $u(t)$ of (2.2) then

$$
\left\|u(t)-u_{n}(t)\right\|=O\left(n^{-1 / 2}\right), n \rightarrow \infty,
$$

uniformly on $t$ in compacts of $\mathbb{R}_{+}$. If $A \in \mathcal{C}(M, 0)$ then the more precise estimate

$$
\left\|u(t)-u_{n}(t)\right\| \leq C(\alpha) M n^{-1 / 2} t^{\alpha}\|A x\|, x \in D(A),
$$

holds, where $C(\alpha)$ depends only on $\alpha$.

Proof: We start from (2.48). Let us find a bound for $S_{\alpha}(t) x-S_{\alpha}(s) x$. Applying $D_{t}^{1} J_{t}^{\alpha}$ to both sides of the equation in (2.2) and using the property (1.21) we obtain

$$
S_{\alpha}^{\prime}(t) x=D_{t}^{1} J_{t}^{\alpha} A S_{\alpha}(t) x=D_{t}^{1} J_{t}^{1} J_{t}^{\alpha-1} S_{\alpha}(t) A x=J_{t}^{\alpha-1} S_{\alpha}(t) A x .
$$

Therefore $S_{\alpha}(t) x-S_{\alpha}(s) x=\int_{s}^{t} P_{\alpha}(\tau) A x d \tau$ with

$$
P_{\alpha}(t)=\int_{0}^{t} g_{\alpha-1}(t-s) S_{\alpha}(s) d s
$$

From $\left\|S_{\alpha}(t)\right\| \leq M e^{\omega t}$ it follows

$$
\left\|P_{\alpha}(t)\right\| \leq M e^{\omega t} g_{\alpha}(t)
$$

Therefore

$$
\left\|S_{\alpha}(t) x-S_{\alpha}(s) x\right\| \leq M|t-s| \max _{\tau \in[s, t]} g_{\alpha}(\tau) e^{\omega \tau}\|A x\|=M\|A x\|\left\{\begin{array}{cc}
(t-s) g_{\alpha}(t) e^{\omega t}, & t \geq s \\
(s-t) g_{\alpha}(s) e^{\omega s}, & s \geq t
\end{array}\right.
$$


Inserting these bounds in (2.48) we get

$$
\begin{aligned}
\left\|u(t)-u_{n}(t)\right\| & \leq \frac{M\|A x\|}{(n-m) !}\left(\frac{n}{t}\right)^{n-m+1}\left[\int_{0}^{t} s^{n-m} e^{-n s / t}(t-s) g_{\alpha}(t) e^{\omega t} d s\right. \\
& \left.+\int_{t}^{\infty} s^{n-m} e^{-n s / t}(s-t) g_{\alpha}(s) e^{\omega s} d s\right] \\
& =\frac{M\|A x\| t^{\alpha} e^{\omega t}}{\Gamma(\alpha)(n-m) !}\left[\gamma(n-m+1, n)-\frac{1}{n} \gamma(n-m+2, n)\right. \\
& +\frac{n^{n-m+1} e^{-\omega t}}{(n-\omega t)^{n-m+\alpha+1}} \Gamma(n-m+\alpha+1, n-\omega t) \\
& \left.-\frac{n^{n-m+1} e^{-\omega t}}{(n-\omega t)^{n-m+\alpha}} \Gamma(n-m+\alpha, n-\omega t)\right]
\end{aligned}
$$

where $\gamma(a, b)=\int_{0}^{b} e^{-t} t^{a-1} d t, \Gamma(a, b)=\int_{b}^{\infty} e^{-t} t^{a-1} d t$ are the incomplete Gamma functions (see [32], vol.1). Using the identities

$$
\gamma(a+1, b)=a \gamma(a, b)-b^{a} e^{-b}, \Gamma(a+1, b)=a \Gamma(a, b)+b^{a} e^{-b},
$$

we simplify the last expression and obtain

$$
\begin{gathered}
\left\|u(t)-u_{n}(t)\right\| \leq \frac{M\|A x\| t^{\alpha} e^{\omega t}}{\Gamma(\alpha)(n-m) !}\left[\frac{m-1}{n} \gamma(n-m+1, n)+\right. \\
\left.e^{-\omega t}\left(1-\frac{\omega t}{n}\right)^{-n} \frac{n^{1-m}(\alpha+\omega t-m)}{(n-\omega t)^{\alpha-m+1}} \Gamma(n-m+\alpha, n-\omega t)+\frac{2 n-\omega t}{n-\omega t} n^{n-m} e^{-n}\right] .
\end{gathered}
$$

Applying the inequalities

$$
\gamma(n+1, n)<\Gamma(n+1)=n !, \Gamma(n+\alpha, n-\omega t)<\Gamma(n+\alpha), 2(n !)^{-1} n^{n} e^{-n} \leq(2 / \pi n)^{1 / 2}
$$

and the asymptotic property of the Gamma functions

$$
\Gamma(n+\alpha) / n !=n^{\alpha-1}\left(1+O\left(n^{-1}\right)\right), \quad n \rightarrow \infty,
$$

we see that the last term in the brackets is dominating as $n \rightarrow \infty$, that implies (2.50). In case $\omega=0$ the estimate $(2.56)$ reduces to

$$
\left\|u(t)-u_{n}(t)\right\| \leq \frac{M\|A x\| t^{\alpha}}{\Gamma(\alpha)(n-m) !}\left[\frac{m-1}{n} \gamma(n+1, n)+\frac{\alpha-m}{n^{\alpha}} \Gamma(n+\alpha, n)+2 n^{n-m} e^{-n}\right] .
$$

This by remarks above implies (2.51)

\subsection{Perturbation properties}

Since in this section we consider solution operators with different generators, we use the notation $S_{\alpha}(t ; A)$ for the solution operators generated by $A$. 
A classical result (see [62] and [60]) is: if $A$ is the generator of a $C_{0}$-semigroup (or COF) and $B \in \mathcal{B}(X)$, then $A+B$ is again a generator of a $C_{0}$-semigroup (COF). This is not true in general for solution operators of (2.2) with $0<\alpha<1$, as the following example shows.

Example 2.24 Let $0<\alpha<1$ be fixed. Assume $X=l^{1}$, the Banach space of all sequences $x=\left\{x_{n}\right\}_{n=1}^{\infty}, x_{n} \in \mathbb{C}$, with norm $\|x\|_{l^{1}}=\sum_{n=1}^{\infty}\left|x_{n}\right|<\infty$. Let $A_{\alpha}$ be an operator defined by

$$
D\left(A_{\alpha}\right)=\left\{x \in l^{1}: \sum_{n=1}^{\infty} n\left|x_{n}\right|<\infty\right\}, \quad A_{\alpha} x=\left\{e^{i \alpha \frac{\pi}{2}} n x_{n}\right\}_{n=1}^{\infty} .
$$

We shall prove that $A_{\alpha} \in \mathcal{C}^{\alpha}$, but $A_{\alpha}+I \notin \mathcal{C}^{\alpha}$.

Applying (1.25), it follows that if $S_{\alpha}\left(t ; A_{\alpha}\right)$ exists, it is given by the formula

$$
S_{\alpha}\left(t ; A_{\alpha}\right) x=\left\{E_{\alpha}\left(e^{i \alpha \frac{\pi}{2}} n t^{\alpha}\right) x_{n}\right\}_{n=1}^{\infty} .
$$

Since $\arg \left(e^{i \alpha \frac{\pi}{2}} n t^{\alpha}\right)=\alpha \pi / 2$ for $t \geq 0$, the asymptotic property of Mittag-Leffler functions (1.27) implies $\left|E_{\alpha}\left(e^{i \alpha \frac{\pi}{2}} n t^{\alpha}\right)\right| \simeq 1 / \alpha, n \rightarrow \infty$. Therefore $\left\|S_{\alpha}\left(t ; A_{\alpha}\right) x\right\|_{l^{1}}<(1 / \alpha+\varepsilon)\|x\|_{l^{1}}$ for some $\varepsilon>0$ and any $x \in l^{1}$, hence $A_{\alpha} \in \mathcal{C}^{\alpha}$.

Similarly, if $S_{\alpha}\left(t ; A_{\alpha}+I\right)$ exists, it is given by

$$
S_{\alpha}\left(t ; A_{\alpha}+I\right) x=\left\{E_{\alpha}\left(\left(e^{i \alpha \frac{\pi}{2}} n+1\right) t^{\alpha}\right) x_{n}\right\}_{n=1}^{\infty} .
$$

Using again the asymptotic relation (1.27),

$$
\left|E_{\alpha}\left(\left(e^{i \alpha \frac{\pi}{2}} n+1\right) t^{\alpha}\right)\right| \simeq \frac{1}{\alpha} \exp \left(\operatorname{Re}\left(e^{i \alpha \frac{\pi}{2}} n+1\right)^{1 / \alpha} t\right), n \rightarrow \infty,
$$

we shall show that, given $t>0$, there is no constant $C$ such that

$$
\left\|S_{\alpha}\left(t ; A_{\alpha}+I\right) x\right\|_{l^{1}} \leq C\|x\|_{l^{1}} .
$$

Indeed, let us use the representation

$$
\operatorname{Re}\left(\left(e^{i \alpha \frac{\pi}{2}} n+1\right)^{1 / \alpha}\right)=\operatorname{Re}\left(\left(r_{n} \exp \left(i \theta_{n}\right)\right)^{1 / \alpha}\right)=r_{n}^{1 / \alpha} \cos \left(\theta_{n} / \alpha\right),
$$

where

$$
r_{n}=\left(n^{2}+1+2 n \cos (\alpha \pi / 2)\right)^{1 / 2}, \theta_{n}=\arctan \frac{n \sin (\alpha \pi / 2)}{1+n \cos (\alpha \pi / 2)} .
$$

Now we study the asymptotic behaviour of $\cos \left(\theta_{n} / \alpha\right)$ as $n \rightarrow \infty$. Using a well-known school formula and (2.59) we obtain

$$
\tan \left(\alpha \pi / 2-\theta_{n}\right)=\frac{\tan (\alpha \pi / 2)-\tan \theta_{n}}{1+\tan (\alpha \pi / 2) \tan \theta_{n}}=\frac{\sin (\alpha \pi / 2)}{n+\cos (\alpha \pi / 2)}=O\left(\frac{1}{n}\right), \quad n \rightarrow \infty,
$$


that is, $\pi / 2-\theta_{n} / \alpha=O(1 / n), n \rightarrow \infty$. Hence $\cos \left(\theta_{n} / \alpha\right)=\sin \left(\pi / 2-\theta_{n} / \alpha\right)=$ $O(1 / n), n \rightarrow \infty$. Together with $r_{n}^{1 / \alpha}=O\left(n^{1 / \alpha}\right), n \rightarrow \infty$, we obtain

$$
r_{n}^{1 / \alpha} \cos \left(\theta_{n} / \alpha\right)=O\left(n^{1 / \alpha-1}\right), n \rightarrow \infty .
$$

Since $1 / \alpha-1>0$, using (2.57) and (2.58), we have the desired result.

If $A \in \mathcal{A}^{\alpha}$, then perturbations by bounded operators are possible also for $\alpha \in(0,1)$. Next we prove a stronger result, which is a generalization of the corresponding result for analytic semigroups ([62], p. 80).

Theorem 2.25 Let $\alpha \in(0,2)$ and $A \in \mathcal{A}^{\alpha}\left(\theta_{0}, \omega_{0}\right)$. Let $B$ be a closed linear operator satisfying $D(B) \supset D(A)$ and

$$
\|B x\| \leq a\|A x\|+b\|x\|, \quad x \in D(A) .
$$

There exists $\delta>0$ such that if $0 \leq a \leq \delta$ then $A+B \in \mathcal{A}^{\alpha}$.

Proof: Since $A \in \mathcal{A}^{\alpha}\left(\theta_{0}, \omega_{0}\right)$, then $\lambda^{\alpha} \in \rho(A)$ for each $\lambda \in \Sigma_{\theta_{0}+\pi / 2}\left(\omega_{0}\right)$. Fix $\omega>\omega_{0}$, $\theta<\theta_{0}$. Then there is a constant $C=C(\theta, \omega)$ such that

$$
\left\|\lambda^{\alpha-1} R\left(\lambda^{\alpha}, A\right)\right\| \leq \frac{C}{|\lambda-\omega|}, \lambda \in \Sigma_{\theta+\pi / 2}(\omega) .
$$

This together with (2.60) implies that for every $x \in X$,

$$
\begin{aligned}
\left\|B R\left(\lambda^{\alpha}, A\right) x\right\| & \leq a\left\|A R\left(\lambda^{\alpha}, A\right) x\right\|+b\left\|R\left(\lambda^{\alpha}, A\right) x\right\| \\
& \leq a\left(1+\frac{C|\lambda|}{|\lambda-\omega|}\right)\|x\|+\frac{b C}{|\lambda-\omega|}\|x\| .
\end{aligned}
$$

Choosing $\lambda$ such that $|\lambda-\omega|>2 b C$ and $\delta=\frac{1}{2}\left(1+C_{1}\right)^{-1}$, where

$$
C_{1}:=\sup _{\lambda \in \Sigma_{\theta+\pi / 2}(\omega),|\lambda-\omega|>1} \frac{C|\lambda|}{|\lambda-\omega|}
$$

we obtain $\left\|B R\left(\lambda^{\alpha}, A\right)\right\|<1$ for $\lambda \in \Sigma_{\theta+\pi / 2}(\omega),|\lambda-\omega|>\max \{1,2 b C\}$. Therefore for such $\lambda$ the operator $I-B R\left(\lambda^{\alpha}, A\right)$ is invertible and

$$
R\left(\lambda^{\alpha}, A+B\right)=R\left(\lambda^{\alpha}, A\right)\left(I-B R\left(\lambda^{\alpha}, A\right)\right)^{-1},
$$

which implies

$$
\left\|\lambda^{\alpha-1} R\left(\lambda^{\alpha}, A+B\right)\right\| \leq \frac{C^{\prime}}{|\lambda-\omega|}, \quad \lambda \in \Sigma_{\theta+\pi / 2}(\omega)
$$

Hence $A+B \in \mathcal{A}^{\alpha}\left(\theta_{0}, \omega_{0}\right)$.

If $A \in \mathcal{C}^{\alpha}, \alpha \in(1,2)$ and $B \in \mathcal{B}(X)$, then $A+B \in \mathcal{C}^{\alpha}$. In the next theorem we prove this in a more general case of bounded time-dependent perturbations $B$. For $\alpha=2$ an analogous theorem is presented in [52]. 
Theorem 2.26 Let $\alpha \in(1,2), A \in \mathcal{C}^{\alpha}(M, \omega)$ and $B(t) \in C\left(\mathbb{R}_{+}, \mathcal{B}(X)\right)$. Then for every $x \in D(A)$ the Cauchy problem

$$
\begin{gathered}
\mathbf{D}_{t}^{\alpha} u(t)=(A+B(t)) u(t), t>0, \\
u(0)=x, u^{\prime}(0)=0,
\end{gathered}
$$

admits an uniquely determined solution $u(t)$ given by the formula

$$
u(t)=S_{\alpha}(t ; A+B) x=\sum_{n=0}^{\infty} S_{\alpha, n}(t ; A) x
$$

where

$$
\begin{gathered}
S_{\alpha, 0}(t ; A)=S_{\alpha}(t ; A) \\
S_{\alpha, n}(t ; A)=\int_{0}^{t} P_{\alpha}(t-s ; A) B(s) S_{\alpha, n-1}(s ; A) d s, \quad n \in \mathbb{N}
\end{gathered}
$$

and $P_{\alpha}(t ; A)$ is defined by (2.53). Moreover, if $K_{t}=\max _{s \in[0, t]}\|B(s)\|$, we have for all $t \in[0, T]$ the bounds

$$
\begin{gathered}
\|u(t)\| \leq M e^{\omega t} E_{\alpha}\left(M K_{T} t^{\alpha}\right)\|x\| \\
\left\|u(t)-S_{\alpha}(t ; A) x\right\| \leq M e^{\omega t}\left(E_{\alpha}\left(M K_{T} t^{\alpha}\right)-1\right)\|x\| .
\end{gathered}
$$

Proof: Applying (2.54) and the semigroup property (1.8) to (2.65), we obtain inductively

$$
\left\|S_{\alpha, n}(t ; A)\right\| \leq M^{n+1} e^{\omega t} K_{t}^{n} g_{\alpha n+1}(t), \quad n \in \mathbb{N}_{0} .
$$

From these bounds it follows that the series representing $S_{\alpha}(t ; A+B)$ in $(2.64)$ are uniformly convergent on compact subsets of $\mathbb{R}_{+}$with respect to the operator norm topology. Hence, $S_{\alpha}(t ; A+B)$ is a strongly continuous function on $\mathbb{R}_{+}$with values in $\mathcal{B}(X)$.

Next we prove that $u(t)$ satisfies (2.62) and (2.63). Since $S_{\alpha}(0 ; A)=I, S_{\alpha, n}(0 ; A)=0, n \in$ $\mathbb{N}, S_{\alpha, n}^{\prime}(0 ; A)=0, n \in \mathbb{N}_{0}$, we have $S_{\alpha}(0 ; A+B)=I, S_{\alpha}^{\prime}(0 ; A+B)=0$, i.e. the initial conditions are satisfied. Applying (2.64) and (2.65), it follows

$$
\begin{aligned}
u(t) & =S_{\alpha}(t ; A) x+\sum_{n=0}^{\infty} \int_{0}^{t} P_{\alpha}(t-s ; A) B(s) S_{\alpha, n}(s ; A) x d s \\
& =S_{\alpha}(t ; A) x+\int_{0}^{t} P_{\alpha}(t-s ; A) B(s) u(s) d s,
\end{aligned}
$$

where the interchanging of the summation and integration is justified by the uniform convergence of the series. Integrodifferentiating (2.68), we obtain

$$
\mathbf{D}_{t}^{\alpha} u(t)=A S_{\alpha}(t ; A) x+\mathbf{D}_{t}^{\alpha} \int_{0}^{t} P_{\alpha}(t-s ; A) B(s) u(s) d s .
$$


Setting $h(t):=B(t) u(t)$ and using (1.20), (1.12), $\left(P_{\alpha} * h\right)(0)=\left(P_{\alpha} * h\right)^{\prime}(0)=0$, the associativity of the convolution, implying $J_{t}^{\beta}(f * g)=\left(J_{t}^{\beta} f\right) * g$, and the semigroup property (1.10), we obtain

$$
\begin{gathered}
\mathbf{D}_{t}^{\alpha}\left(P_{\alpha}(. ; A) * h\right)=D_{t}^{\alpha}\left(P_{\alpha}(. ; A) * h\right)=D_{t}^{2} J_{t}^{2-\alpha}\left(J_{t}^{\alpha-1} S_{\alpha}(. ; A) * h\right) \\
=D_{t}^{1}\left(S_{\alpha}(. ; A) * h\right)=S_{\alpha}^{\prime}(t ; A) * h+S_{\alpha}(0 ; A) h .
\end{gathered}
$$

Since $S^{\prime}(0 ; A)=0$, it follows by the closedness of $A$

$$
\begin{gathered}
S_{\alpha}^{\prime}(t ; A)=J_{t}^{1} D_{t}^{2} S_{\alpha}(t ; A)=J_{t}^{\alpha-1} J_{t}^{2-\alpha} D_{t}^{2} S_{\alpha}(t ; A) \\
=J_{t}^{\alpha-1} \mathbf{D}_{t}^{\alpha} S_{\alpha}(t ; A)=J_{t}^{\alpha-1} A S_{\alpha}(t ; A)=A J_{t}^{\alpha-1} S_{\alpha}(t ; A)=A P_{\alpha}(t ; A) .
\end{gathered}
$$

Combining (2.68), (2.69), (2.70) and (2.71), we obtain that $u(t)$ satisfies (2.62).

To prove the uniqueness, let $v: \mathbb{R}_{+} \rightarrow D(A)$ be a solution of $(2.62)$ with $v(0)=v^{\prime}(0)=0$. Then (1.21) gives $v(t)=J_{t}^{\alpha} A v(t)+J_{t}^{\alpha} B(t) v(t)$ and applying the variation of parameters formula (see [64], Prop. 1.2), $v(t)$ satisfies the integral equation

$$
v(t)=\int_{0}^{t} P_{\alpha}(t-s ; A) B(s) v(s) d s .
$$

Setting $m_{t}=\max _{s \in[0, t]}\|v(s)\|$, we see that for $m_{t}>0$

$$
m_{t} \leq \frac{M K_{t} m_{t}}{\Gamma(\alpha)} \int_{0}^{t}(t-s)^{\alpha-1} e^{\omega(t-s)} d s \leq \frac{M K_{t} m_{t}}{\Gamma(\alpha+1)} t^{\alpha} e^{\omega t}<m_{t},
$$

if $t>0$ is chosen sufficiently small. Thus, $v(t)=0$ on $\left[0, t_{0}\right]$ with $t_{0}>0$. Iteration of this argument leads to $v(t)=0$ on $\mathbb{R}_{+}$. The bounds (2.66) follow directly from (2.67). 


\section{Chapter 3}

\section{Subordination principle}

The subordination principle, presented earlier by Prüss [64] for general Volterra integral equations, is studied in detail for the fractional evolution equation. Besides some additional properties of the subordinated solution operator, we discuss also some new aspects of this principle, such as invertibility, an inversion formula and the semigroup of subordination operators.

\subsection{Subordination principle and its invertibility}

Let $\alpha \in(0,2], m=\lceil\alpha\rceil$ and $\mathbf{D}_{t}^{\alpha}$ be the Caputo fractional derivative of order $\alpha$. Let $A$ be a linear closed densely defined operator in a Banach space $X$ and $x \in X$. In this section we are concerned again with the fractional evolution equation

$$
\mathbf{D}_{t}^{\alpha} u(t)=A u(t), \quad u(0)=x, u^{(k)}(0)=0, k=1, \ldots, m-1 .
$$

It is well known ([34], p.169) that if $A$ generates a cosine operator family $S_{2}(t)$ then $A$ generates a $C_{0}$-semigroup $S_{1}(t)$ and they are related by the abstract Weierstrass formula:

$$
S_{1}(t)=\frac{1}{\sqrt{\pi t}} \int_{0}^{\infty} e^{-s^{2} /(4 t)} S_{2}(s) d s, t>0,
$$

which incidentally shows that $S_{1}(t)$ is an analytic semigroup of angle $\pi / 2$. However, the converse is false: there are infinitesimal generators of analytic semigroups of angle $\pi / 2$ which do not generate cosine families. A well-known example is the Laplacian $\Delta$ in $L^{p}\left(\mathbb{R}^{n}\right)$ if $p \neq 2, n \neq 1$ (see e.g. [45]).

Generalizing these classical results, we study the relationship between problems (3.1) for different values of $\alpha$, resulting in the subordination principle. This principle is presented in the next two theorems. The first of them is a particular case of [64], Th. 4.2 and Cor. 4.5. Here we give another proof based on the properties of Mittag-Leffler and Wright functions. 
Theorem 3.1 Let $0<\alpha<\beta \leq 2, \gamma=\alpha / \beta, \omega \geq 0$. If $A \in \mathcal{C}^{\beta}(\omega)$ then $A \in \mathcal{C}^{\alpha}\left(\omega^{1 / \gamma}\right)$ and the following representation holds

$$
S_{\alpha}(t):=\int_{0}^{\infty} \varphi_{t, \gamma}(s) S_{\beta}(s) d s, \quad t>0,
$$

where $\varphi_{t, \gamma}(s):=t^{-\gamma} \Phi_{\gamma}\left(s t^{-\gamma}\right)$ and $\Phi_{\gamma}(z)$ is defined by (1.30); (3.3) holds in the strong sense.

Proof: Define

$$
S(t) x=\int_{0}^{\infty} \varphi_{t, \gamma}(s) S_{\beta}(s) x d s, \quad t>0 .
$$

Our aim is to prove that $S(t)$ satisfies the conditions of Theorem 2.9 with $\omega^{1 / \gamma}$ instead of $\omega$. Since $A \in \mathcal{C}^{\beta}(\omega)$, then $\left(\omega^{\beta}, \infty\right) \subset \varrho(A)$ and there is a constant $M \geq 1$ such that

$$
\left\|S_{\beta}(s)\right\| \leq M e^{\omega s}, \quad s \geq 0 .
$$

Then the condition $\left(\left(\omega^{1 / \gamma}\right)^{\alpha}, \infty\right) \subset \varrho(A)$ is trivially fulfilled. Further, (3.5) together with (1.31) and (1.32) implies

$$
\|S(t)\| \leq \int_{0}^{\infty} \varphi_{t, \gamma}(s)\left\|S_{\beta}(s)\right\| d s \leq M \int_{0}^{\infty} \varphi_{t, \gamma}(s) e^{\omega s} d s=M E_{\gamma}\left(\omega t^{\gamma}\right), t \geq 0 .
$$

This together with (2.9) gives

$$
\|S(t)\| \leq M C \exp \left(\omega^{1 / \gamma} t\right), \quad t \geq 0 .
$$

Next we prove the strong continuity of $S(t)$ at the origin on the basis of the dominated convergence theorem, using (1.32), (1.33), (3.7) and the strong continuity of $S_{\beta}(t)$ at the origin:

$$
\lim _{t \downarrow 0} S(t) x=\lim _{t \downarrow 0} \int_{0}^{\infty} \Phi_{\gamma}(\sigma) S_{\beta}\left(\sigma t^{\gamma}\right) x d \sigma=\int_{0}^{\infty} \Phi_{\gamma}(\sigma) x d \sigma=x .
$$

For $\lambda>\omega^{1 / \gamma}$, using (3.4) and interchanging the order of integration, we have

$$
\int_{0}^{\infty} e^{-\lambda t} S(t) x d t=\int_{0}^{\infty} S_{\beta}(s) x \int_{0}^{\infty} e^{-\lambda t} \varphi_{t, \gamma}(s) d t d s
$$

Substituting $\mu=t \tau$ in (1.30) and shifting the new contour $\Gamma^{\prime}=\Gamma / t$ to $\Gamma$ we get the integral representation

$$
\varphi_{t, \gamma}(s)=\frac{1}{2 \pi i} \int_{\Gamma} \tau^{\gamma-1} \exp \left(\tau t-\tau^{\gamma} s\right) d \tau
$$

Therefore

$$
\int_{0}^{\infty} e^{-\lambda t} \varphi_{t, \gamma}(s) d t=\lambda^{\gamma-1} \exp \left(-\lambda^{\gamma} s\right)
$$

Using now (2.6) for $S_{\beta}(s)$ we obtain from (3.8) and (3.10)

$$
\int_{0}^{\infty} e^{-\lambda t} S(t) x d t=\lambda^{\gamma-1} \int_{0}^{\infty} \exp \left(-\lambda^{\gamma} s\right) S_{\beta}(s) x d s=\lambda^{\alpha-1} R\left(\lambda^{\alpha}, A\right) x
$$


So, we have proved the conditions of Theorem 2.9. Therefore $A \in \mathcal{C}^{\alpha}\left(\omega^{1 / \gamma}\right)$ and the corresponding solution operator $S_{\alpha}(t)=S(t)$.

Since $\Phi_{1 / 2}(z)=\pi^{-1 / 2} e^{-z^{2} / 4}$, the formula (3.3) coincides with the abstract Weierstrass formula (3.2) when $\alpha=1, \beta=2$. By (3.6) we obtain the following additional result:

Corollary 3.2 Assume $\alpha \in(0,1), \omega \in \mathbb{R}$, and $A$ is the infinitesimal generator of a $C_{0}$-semigroup $S_{1}(t)$ satisfying $\left\|S_{1}(t)\right\| \leq M e^{\omega t}, t \geq 0$. Then $A \in \mathcal{C}^{\alpha}$ and

$$
\left\|S_{\alpha}(t)\right\| \leq M E_{\alpha}\left(\omega t^{\alpha}\right), t \geq 0
$$

Next we study the analyticity properties of the subordinated resolvent $S_{\alpha}(t)$.

Theorem 3.3 Under the hypotheses of Theorem 3.1 the solution operator $S_{\alpha}(t)$ has the following additional properties, where $\theta(\gamma)=(1 / \gamma-1) \pi / 2$ :

(a) $S_{\alpha}(t)$ admits an analytic extension to the sector $\Sigma_{\min \{\theta(\gamma), \pi\}}$;

(b) If $\omega=0,\left\|S_{\alpha}(t)\right\| \leq C$ for $t \in \Sigma_{\min \{\theta(\gamma), \pi\}-\varepsilon}$, where $C=C(\gamma, \varepsilon)$;

(c) If $\omega>0,\left\|S_{\alpha}(t)\right\| \leq C e^{\delta \operatorname{Re} t}$ for $t \in \Sigma_{\min \{\theta(\gamma), \pi / 2\}-\varepsilon}$, where $\delta=\delta(\gamma, \varepsilon), C=C(\delta, \gamma, \varepsilon)$.

Proof: Let

$$
\left\|S_{\beta}(t)\right\| \leq M e^{\omega t}, t \geq 0
$$

and $\Sigma$ be the sector $\Sigma=\Sigma_{\min \{\theta(\gamma), \pi\}}$. The function under the integral sign in (3.3) is analytic in $t \in \Sigma$. In view of the asymptotic expansion (1.33) and the inequality (3.11) and, noting that $\operatorname{Re}\left(t^{-\gamma /(1-\gamma)}\right)>0$ when $t \in \Sigma$ and $1 /(1-\gamma)>1$ for $0<\gamma<1$, it follows that the integral in (3.3) is absolutely and uniformly convergent on compact subsets of $\Sigma$. Therefore, $S_{\alpha}(t)$ given by (3.3) is analytic in $\Sigma$ (see [57], p. 32, Th. 7). This implies (a). In what follows, $c_{n}$ denote positive constants, not depending on $t$. Let $t \in \Sigma_{\min \{\theta(\gamma), \pi\}-\varepsilon}$. Then (3.3) and (3.11) imply

$$
\left\|S_{\alpha}(t)\right\| \leq M|t|^{-\gamma} \int_{0}^{\infty}\left|\Phi_{\gamma}\left(s t^{-\gamma}\right)\right| e^{\omega s} d s=M \int_{0}^{\infty}\left|\Phi_{\gamma}\left(s e^{i \varphi}\right)\right| e^{\left.\omega|t|\right|^{\gamma} s} d s,
$$

where $\varphi=-\gamma \arg t$, that is $|\varphi| \leq(1-\gamma) \pi / 2-\gamma \varepsilon$. Thanks to the expansion (1.33) there exists $S>0$ such that for any $s>S$

$$
\left|\Phi_{\gamma}\left(s e^{i \varphi}\right)\right| \leq c_{0} s^{(\gamma-1 / 2) /(1-\gamma)} \exp \left\{-c_{1} \sin \frac{\gamma \varepsilon}{1-\gamma} s^{1 /(1-\gamma)}\right\} .
$$

Divide the last integral in (3.12) into two parts: $I_{1}$ from 0 to $S$ and $I_{2}$ from $S$ to $\infty$. If $\omega=0$ then $I_{1}$ and $I_{2}$ are uniformly bounded: $I_{1}$ - since $\Phi_{\gamma}(z)$ is an entire function and $I_{2^{-}}$ in view of (3.13). Hence b) is proven. 
Assume now $\omega>0$ and $t \in \Sigma_{\min \{\theta(\gamma), \pi / 2\}-\varepsilon}$. Denote $r=|t|$. Then $I_{1} \leq c_{2} \exp \left(c_{3} r^{\gamma}\right) \leq$ $c_{4} \exp \left(c_{3} r\right)$, since $0<\gamma<1$. Applying (3.13) we have

$$
I_{2} \leq M c_{0} \int_{0}^{\infty} s^{(\gamma-1 / 2) /(1-\gamma)} \exp \left\{-c_{1} \sin \frac{\gamma \varepsilon}{1-\gamma} s^{1 /(1-\gamma)}+\omega r^{\gamma} s\right\} d s .
$$

Using now the asymptotic expansion (see [33], p. 141)

$$
\int_{0}^{\infty} s^{p} \exp \left(-s^{q}+y s\right) d s \approx A y^{(2 p-q+2) /(2 q-2)} \exp \left\{a y^{q /(q-1)}\right\}, \quad q>1, y \rightarrow+\infty,
$$

where $A=A(p, q), a=a(q)$ are positive constants, we obtain $I_{2} \leq c_{5} r^{c_{6}} \exp \left(c_{7} r\right) \leq$ $c_{8} \exp \left(c_{9} r\right)$. Then $I_{1}+I_{2} \leq C \exp \left(c_{10} r\right)$. Since $|\arg t|<\pi / 2-\varepsilon$, we can take $\delta=c_{10} / \sin \varepsilon$ and then $\left\|S_{\alpha}(t)\right\| \leq C \exp (\delta \operatorname{Re} t)$.

So, we proved in Theorem 3.3 that $S_{\alpha}(t)$ is an analytic solution operator. Note that if $\omega=0$ and $\gamma<1 / 2, S_{\alpha}(t)$ is in fact analytic in a sector with opening angle $\theta>\pi$ and if $\gamma<1 / 3$, it is analytic in all of $\mathbb{C}$ except of a neighbourhood of the negative real axis. To complete the picture, we note in addition to Theorem 3.3 that if $S_{\beta}(t)$ is analytic in $\Sigma_{\theta_{0}}$ and $\alpha=\gamma \beta, \gamma<1$, then $S_{\alpha}(t)$ is analytic in $\Sigma_{\min \left\{\theta(\gamma)+\theta_{0} / \gamma, \pi / 2\right\}}$. Indeed, if for $|\phi|<\theta_{0}$ we consider the path $\Gamma_{R}=[0, R], R \exp (i[0, \phi]),[R, 0] e^{i \phi}$, then by Cauchy's theorem $\int_{\Gamma_{R}} \varphi_{t, \gamma}(z) S_{\beta}(z) d z=0$, i.e. for $R \rightarrow \infty$ we obtain

$$
S_{\alpha}(t)=\int_{0}^{\infty} \varphi_{t, \gamma}(s) S_{\beta}(s) d s=\int_{0}^{\infty} \varphi_{t, \gamma}\left(s e^{i \phi}\right) S_{\beta}\left(s e^{i \phi}\right) e^{i \phi} d s
$$

and the desired result follows as in the proof of Theorem 3.3. This result can be also obtained applying Theorem 2.14.

Next we present some simple but interesting corollaries of the above theorems.

Corollary 3.4 If $A \in \mathcal{C}^{\alpha}(1,0)$ for some $\alpha \in(0,1]$, then $A \in \mathcal{C}^{\alpha}(1,0)$ for all $\alpha \in(0,1]$.

Proof: If $A \in \mathcal{C}^{\alpha}(1,0)$ for some $\alpha \in(0,1]$, then according to Theorem $2.8,(0, \infty) \subseteq \varrho(A)$ and (2.12) holds with $\omega=0, M=1$ and $n=0$, that is $\left\|\lambda^{\alpha-1} R\left(\lambda^{\alpha}, A\right)\right\| \leq 1 / \lambda, \lambda>0$. This is exactly $\|R(\mu, A)\| \leq 1 / \mu, \mu>0$. Hence $A \in \mathcal{C}^{1}(1,0)$ and Theorem 3.1 implies $A \in \mathcal{C}^{\alpha}(1,0)$ for all $\alpha \in(0,1]$.

Combining Theorem 3.3 and Corollary 3.4, we obtain the following result:

Corollary 3.5 Let $\alpha \in(0,1)$ and $S_{\alpha}(t)$ be a contraction: $\left\|S_{\alpha}(t)\right\| \leq 1, t \geq 0$. Then $S_{\alpha}(t)$ is necessarily an analytic solution operator.

The next example illustrates how Theorems 3.1 and 3.3 can be applied to obtain solutions of (3.1) for noninteger $\alpha$ from known solutions for $\alpha=1$ or 2 and to study their properties. 
Example 3.6 Consider the fractional diffusion-wave equation (see Example 2.18). It is well-known that this problem is well-posed for $\alpha=2$, more precisely $A=k^{2} D_{x}^{2} \in$ $\mathcal{C}^{2}\left(L^{p}(\mathbb{R}) ; 1,0\right)$, and the solution is given by the d'Alembert formula:

$$
\left(S_{2}(t) f\right)(x)=\frac{1}{2}(f(x+k t)+f(x-k t)) .
$$

Therefore, according to Theorem 3.3 (a),(b), $A \in \mathcal{A}^{\alpha}\left(\frac{2-\alpha}{2 \alpha} \pi, 0\right)$ if $\alpha>\frac{2}{3}, A \in \mathcal{A}^{\alpha}(\pi, 0)$, if $\alpha<\frac{2}{3}$. Applying formulas (3.3) and (3.14), we obtain the explicit form of the solution

$$
\begin{aligned}
S_{\alpha}(t) f(x) & =t^{-\alpha / 2} \int_{0}^{\infty} \Phi_{\alpha / 2}\left(s t^{-\alpha / 2}\right) S_{2}(s) f(x) d s \\
& =\frac{1}{2|k| t^{\alpha / 2}} \int_{-\infty}^{\infty} \Phi_{\alpha / 2}\left(\frac{|s|}{|k| t^{\alpha / 2}}\right) f(x-s) d s .
\end{aligned}
$$

This representation coincides with a result given in [54].

So far, given $A \in \mathcal{C}^{\beta}$, we studied the properties of $S_{\alpha}(t)$ for $\alpha<\beta$. Now we shall try to do the converse: to find properties of $S_{\alpha}(t)$, sufficient for the existing of $S_{\beta}(t), \beta>\alpha$.

Set $\alpha=1$ in Theorem 3.3. If $A \in \mathcal{C}^{\beta}$ for some $\beta \in(1,2]$, then $A$ generates an analytic semigroup $S_{1}(t)$ of angle $(\beta-1) \pi / 2$. We are interested in the opposite: whether the analyticity of $S_{1}(t)$ in $\Sigma_{(\beta-1) \pi / 2}$ suffices for existing of exponentially bounded solution operator $S_{\beta}(t)$ ? For $\beta=2$ the answer is negative, as it was mentioned in the beginning of this section. The next example shows that the same answer holds for $\beta \in(1,2)$.

Example 3.7 Fix $\beta \in(1,2)$ and consider in $X=L^{1}(\mathbb{R})$ the operator $A_{\beta}=e^{i(2-\beta) \pi / 2} D_{x}^{2}$, $D(A)=W^{2,1}(\mathbb{R})$. Since $D_{x}^{2}$ generates a bounded analytic semigroup of angle $\pi / 2$ then $\left\|R\left(\lambda, D_{x}^{2}\right)\right\|_{\mathcal{B}\left(L^{1}\right)} \leq M /|\lambda|, \quad \lambda \in \Sigma_{\pi-\varepsilon}$. Therefore

$$
\left\|R\left(\lambda, A_{\beta}\right)\right\|_{\mathcal{B}\left(L^{1}\right)}=\left\|R\left(\lambda e^{-i(2-\beta) \pi / 2}, D_{x}^{2}\right)\right\|_{\mathcal{B}\left(L^{1}\right)} \leq M /|\lambda|, \quad \lambda \in \Sigma_{\beta \pi / 2-\varepsilon},
$$

i.e. $A_{\beta}$ generates a bounded analytic semigroup of angle $(\beta-1) \pi / 2$. Let us now consider the problem

$$
\mathbf{D}_{t}^{\beta} u(x, t)=A_{\beta} u(x, t), u(x, 0)=f(x), u_{t}(x, 0)=0,
$$

with $f \in \mathcal{S}(\mathbb{R})$, the space of rapidly decreasing functions of Schwartz. Then the solution is given by

$$
u(x, t)=\frac{1}{2} e^{-i(2-\beta) \pi / 4} \int_{-\infty}^{\infty} \varphi_{t, \beta / 2}\left(e^{-i(2-\beta) \pi / 4}|s|\right) f(x-s) d s .
$$

This can be verified proving that $\int_{0}^{\infty} e^{-\lambda t} u(x, t) d t=\lambda^{\beta-1} R\left(\lambda^{\beta}, A_{\beta}\right) f(x)$. Suppose $A_{\beta} \in$ $\mathcal{C}^{\beta}$ for $X=L^{1}(\mathbb{R})$. Then given $t>0$ there is a constant $C$ such that $\|u(x, t)\|_{L^{1}} \leq C\|f\|_{L^{1}}$ for any function of the form (3.15). Let $\delta_{n}$ be a delta sequence (i.e. for any $y \in L^{1}(\mathbb{R})$, $\delta_{n} * y \rightarrow y$ with respect to \|\|$_{L^{1}}$ as $n \rightarrow \infty$ and $\left.\left\|\delta_{n}\right\|_{L^{1}}=1\right)$. Setting $f(x)=\delta_{n}$ in (3.15) and calling $u_{n}(x, t)$ the function so obtained we see that

$$
u_{n}(x, t) \rightarrow k(x, t)=\frac{1}{2} e^{-i(2-\beta) \pi / 4} \varphi_{t, \beta / 2}\left(e^{-i(2-\beta) \pi / 4}|x|\right)
$$

for all $x \in \mathbb{R}$. Since $\left\|\delta_{n}\right\|_{L^{1}}=1$, it follows from Fatou's lemma that $k(\cdot, t)$ must be in $L^{1}(\mathbb{R})$ which is false in view of the asymptotic expansion (1.33). 
Next we give a simple sufficient condition, under which $A \in \mathcal{C}^{\beta}(0)$.

Proposition 3.8 If $1<\beta<2$ and

$$
\|R(\lambda, A)\| \leq M /|\lambda|, \lambda \in \Sigma_{\beta \pi / 2}
$$

then $A \in \mathcal{C}^{\beta}(0)$.

That is, "a little bit more" than analyticity of $S_{1}(t)$ in $\Sigma_{(\beta-1) \pi / 2}$ is sufficient. Condition (3.16) is satisfied for example, if the analytic semigroup $S_{1}(t)$ in $\Sigma_{(\beta-1) \pi / 2}$ admits boundary values [2], that is

$$
\sup _{\Sigma^{\prime}}\left\|S_{1}(t)\right\|<\infty, \quad \text { where } \Sigma^{\prime}:=\left\{t \in \mathbb{C} ; t \in \Sigma_{(\beta-1) \pi / 2}, \operatorname{Im} t \geq 0,|t| \leq 1\right\} .
$$

Proof: The key to the proof of Proposition 3.8 is the following lemma due to Prüss [64], Proposition 0.1:

Lemma 3.9 Let $h(\lambda)$ be analytic for $\operatorname{Re} \lambda>0$ and $C \geq 0$ is such that

$$
\|\lambda h(\lambda)\| \leq C ; \quad\left\|\lambda^{2} h^{\prime}(\lambda)\right\| \leq C, \quad \operatorname{Re} \lambda>0 .
$$

Then

$$
\left\|r^{n+1} h^{(n)}(r) / n !\right\| \leq 2 C, \quad r>0, \quad n \in \mathbb{N} .
$$

Setting now $h(\lambda)=\lambda^{\beta-1} R\left(\lambda^{\beta}, A\right)$ it easily follows from (3.16) that $h(\lambda)$ satisfies (3.17) with $C=\max \left\{M, M(\beta-1)+M^{2} \beta\right\}$. Then we have (3.18), that is exactly (2.12) with $\alpha=\beta, \omega=0$. Hence $A \in \mathcal{C}^{\beta}(0)$.

Corollary 3.10 If $A$ is the infinitesimal generator of a bounded analytic semigroup of angle $(\beta-1) \pi / 2+\varepsilon$, where $\varepsilon \in(0,(2-\beta) \pi / 2)$ can be chosen arbitrarily small, then $A \in \mathcal{C}^{\beta}(0)$.

This corollary incidentally shows that if $A$ generates a bounded analytic semigroup of angle $\pi / 2$ (e.g. the Laplacian $\Delta$ in $L^{p}\left(\mathbb{R}^{n}\right)$ ) then $A \in \mathcal{C}^{\beta}$ for any $\beta<2$, moreover $S_{\beta}(t)$ is analytic in $\Sigma_{\delta_{\beta}}$ for sufficiently small $\delta_{\beta}$. Recall that in this case $A$ does not necessarily generate a cosine family, i.e. not necessarily $A \in \mathcal{C}^{2}$.

We conclude this section with another application of the subordination principle. Let $\alpha \in(1,2)$. If $A_{1}, A_{2} \in \mathcal{C}^{\alpha}$ and they commute, is it true that $\overline{A_{1}+A_{2}} \in \mathcal{C}^{\alpha}$ ? For $\alpha=1$ the answer is positive (see [71]) and for $\alpha=2-$ negative (see [38]). Here we notice only a weaker property of $\overline{A_{1}+A_{2}}$, as follows:

Proposition 3.11 Let $\alpha \in(1,2), A_{1}, A_{2} \in \mathcal{C}^{\alpha}$ and they commute. Then $\overline{A_{1}+A_{2}}$ generates a semigroup analytic in $\Sigma_{(\alpha-1) \pi / 2}$. 
Proof: According to Theorem 3.1, $A_{1}$ and $A_{2}$ generate $C_{0}$-semigroups analytic in $\Sigma_{(\alpha-1) \pi / 2}$ and given by the formulas

$$
S_{1}\left(t ; A_{j}\right) x=\int_{0}^{\infty} \varphi_{t, 1 / \alpha}(s) S_{\alpha}\left(t ; A_{j}\right) x d s, \quad j=1,2 .
$$

Since $S_{\alpha}\left(t ; A_{1}\right)$ and $S_{\alpha}\left(t ; A_{2}\right)$ commute for all $t, s>0$, then $S_{1}\left(t ; A_{1}\right)$ and $S_{2}\left(t ; A_{2}\right)$ commute for $t, s>0$. Hence ([71]) $\overline{A_{1}+A_{2}} \in \mathcal{C}^{1}$ and $S_{1}\left(t ; \overline{A_{1}+A_{2}}\right)=S_{1}\left(t ; A_{1}\right) S_{2}\left(t ; A_{2}\right)$ is a strongly continuous semigroup analytic in $\Sigma_{(\alpha-1) \pi / 2}$.

Let us note that the analyticity of $S_{1}\left(t ; \overline{A_{1}+A_{2}}\right)$ in $\Sigma_{(\alpha-1) \pi / 2}$ does not imply in general $\overline{A_{1}+A_{2}} \in \mathcal{C}^{\alpha}$ ( Example 3.7), but only $\overline{A_{1}+A_{2}} \in \mathcal{C}^{\alpha-\varepsilon}$ for any $\varepsilon>0$.

\subsection{An inversion formula}

Let $X$ be a complex Banach space. We give an inversion of formula (3.3) provided $A$ satisfies some additional conditions. Recall the Mellin transform defined by

$$
\{\mathcal{M} f\}(\varrho)=\int_{0}^{\infty} t^{\varrho-1} f(t) d t
$$

and its inverse

$$
f(t)=\frac{1}{2 \pi i} \int_{c-i \infty}^{c+i \infty}\{\mathcal{M} f\}(\varrho) t^{-\varrho} d \varrho .
$$

From the integral representation (1.30) of $\Phi_{\gamma}(z), \gamma \in(0,1)$, we obtain

$$
\left\{\mathcal{M} \Phi_{\gamma}\right\}(\varrho):=\Gamma(\varrho) / \Gamma(1-\gamma+\gamma \varrho), \operatorname{Re} \varrho>0 .
$$

Taking $c \in(0,1)$ in $(3.19)$ and making the substitution $\sigma=1-\varrho$, we have

$$
\Phi_{\gamma}(t)=\frac{1}{2 \pi i} \int_{L} \frac{\Gamma(1-\sigma)}{\Gamma(1-\gamma \sigma)} t^{\sigma-1} d \sigma
$$

where $L=\{c+i y,-\infty<y<\infty\}$ and $c \in(0,1)$ can be arbitrarily chosen thanks to Cauchy's theorem. Substituting this representation in (3.3) we obtain formally

$$
S_{\alpha}(t)=\frac{1}{2 \pi i} \int_{L} \frac{\Gamma(1-\sigma)}{\Gamma(1-\gamma \sigma)} t^{-\gamma \sigma}\left\{\mathcal{M} S_{\beta}\right\}(\sigma) d \sigma
$$

We are looking now for conditions under which (3.20) holds for $0<\beta \leq 1<\alpha<2$, $\gamma=\alpha / \beta>1$. To this aim we involve fractional powers of positive operators.

If $-A$ is a positive closed densely defined operator, we have (see e.g. [1], eq. (4.6.9)):

$$
(-A)^{-\sigma}=\frac{\sin \pi \sigma}{\pi} \int_{0}^{\infty} s^{-\sigma}(s-A)^{-1} d s, \quad 0<\operatorname{Re} \sigma<1 .
$$


In particular, if $A$ generates an exponentially decaying $C_{0}$-semigroup $S_{1}(t)$, then $(-A)$ is positive and the following representation holds (see e.g. [1], Th. 4.6.6.):

$$
(-A)^{-\sigma}=\frac{1}{\Gamma(\sigma)} \int_{0}^{\infty} t^{\sigma-1} S_{1}(t) d t, \quad \operatorname{Re} \sigma>0
$$

An analogous representation of $(-A)^{-\sigma}$ for $0<\operatorname{Re} \sigma<1$ in terms of the solution operators $S_{\beta}(t), 0<\beta<1$, is given in the next theorem.

Theorem 3.12 Let $0<\beta \leq 1, A \in \mathcal{C}^{\beta}$ and assume that there are constants $M \geq 1$ and $\mu>0$ such that

$$
\left\|S_{\beta}(t)\right\| \leq M E_{\beta}\left(-\mu t^{\beta}\right), \quad t \geq 0 .
$$

Then

$$
(-A)^{-\sigma}=\frac{\beta \Gamma(1-\beta \sigma)}{\Gamma(\sigma) \Gamma(1-\sigma)} \int_{0}^{\infty} t^{\beta \sigma-1} S_{\beta}(t) d t, \quad 0<\operatorname{Re} \sigma<1
$$

Proof: Applying (3.22) it follows

$$
\left\|\int_{0}^{\infty} t^{\beta \sigma-1} S_{\beta}(t) d t\right\| \leq M \int_{0}^{\infty} t^{\beta \operatorname{Re} \sigma-1} E_{\beta}\left(-\mu t^{\beta}\right) d t, \quad 0<\operatorname{Re} \sigma<1,
$$

where the integral on the right is convergent in view of the asymptotic expansion (1.28). Then the right side of (3.23) is a bounded linear operator. According to (2.6)

$$
R\left(s^{\beta}, A\right)=s^{1-\beta} \int_{0}^{\infty} e^{-s t} S_{\beta}(t) d t
$$

and thanks to (3.22) and (1.26) we obtain the estimate

$$
\left\|R\left(s^{\beta}, A\right)\right\| \leq M s^{1-\beta} \int_{0}^{\infty} e^{-s t} E_{\beta}\left(-\mu t^{\beta}\right) d t=M /\left(s^{\beta}+\mu\right), \quad s>0 .
$$

Therefore $(-A)$ is positive and formula (3.21) holds for it. Making the substitution $s=u^{\beta}$ in (3.21), applying (2.6) and the well-known formula $\Gamma(\sigma) \Gamma(1-\sigma)=\pi / \sin \pi \sigma$, it follows by Fubini's theorem

$$
\begin{gathered}
(-A)^{-\sigma}=\frac{\beta}{\Gamma(\sigma) \Gamma(1-\sigma)} \int_{0}^{\infty} u^{-\beta \sigma+\beta-1} R\left(u^{\beta}, A\right) d u= \\
\frac{\beta}{\Gamma(\sigma) \Gamma(1-\sigma)} \int_{0}^{\infty} u^{-\beta \sigma} \int_{0}^{\infty} e^{-u t} S_{\beta}(t) d t d u=\frac{\beta \Gamma(1-\beta \sigma)}{\Gamma(\sigma) \Gamma(1-\sigma)} \int_{0}^{\infty} S_{\beta}(t) t^{\beta \sigma-1} d t .
\end{gathered}
$$

Substituting now (3.23) in (3.20), we obtain

$$
S_{\alpha}(t)=\frac{1}{2 \pi i} \int_{L} \frac{\Gamma(\sigma) \Gamma(1-\sigma)}{\Gamma(1-\alpha \sigma)} t^{-\alpha \sigma}(-A)^{-\sigma} d \sigma .
$$


Let us estimate the expression under the integral sign. The asymptotic expansion of the Gamma function (see [57], p. 49)

$$
|\Gamma(c+i y)|=\sqrt{2 \pi}|y|^{c-1 / 2} \exp \left(-\frac{\pi}{2}|y|\right)(1+O(1 / y)), \quad|y| \rightarrow \infty,
$$

implies that if $\sigma=c+i y,|y| \rightarrow \infty$, then

$$
\left|\frac{\Gamma(\sigma) \Gamma(1-\sigma)}{\Gamma(1-\alpha \sigma)}\right|=\sqrt{2 \pi}|y|^{\alpha c-1 / 2} \exp \left(-(2-\alpha) \frac{\pi}{2}|y|\right)(1+O(1 / y)) .
$$

Further, $(-A)^{-\sigma} x=(-A)^{-c}(-A)^{-i y} x$ for $x \in D\left(A^{2}\right)$ [1], Th.4.6.5. Suppose that $A$ satisfies conditions of the theorem. Then, from (3.23), (3.22) and the denseness of $D\left(A^{2}\right)$ in $X$, it follows $\left\|(-A)^{-\sigma}\right\| \leq\left\|(-A)^{-c}\right\|\left\|(-A)^{-i y}\right\| \leq C(\beta, c)\left\|(-A)^{-i y}\right\|$. Therefore, the integral in (3.24) is convergent if we impose some conditions on the imaginary powers of the operator $(-A)$.

The main result of this section is given in the following

Theorem 3.13 Assume $0<\beta \leq 1<\alpha<2$ and

$$
-A \in \mathcal{B I P}((2-\alpha-\varepsilon) \pi / 2)
$$

for some $\varepsilon \in(0,2-\alpha)$. Then $A \in \mathcal{C}^{\alpha}$ and the representations (3.20) and (3.24) hold.

Proof: According to [65], Theorem 2, (3.26) implies that $A$ generates a bounded analytic semigroup $S_{1}(t)$ of angle $(\alpha-1+\varepsilon) \pi / 2$ and, applying Proposition 3.8, it follows that $A \in \mathcal{C}^{\alpha}$. Moreover, since $0 \in \varrho(A), S_{1}(t)$ is exponentially decaying: there are constants $M \geq 1$ and $\mu>0$ such that $\left\|S_{1}(t)\right\| \leq M e^{-\mu t}, t \geq 0$ (see [62], Theorem 4.3.). Applying Corollary 3.2, it follows that $A$ satisfies the conditions of Theorem 3.12. Therefore the representation (3.23) holds. In view of (3.25) and (3.26) the integral in (3.24) (respectively in $(3.20))$ is absolutely convergent. Set

$$
S(t):=\frac{1}{2 \pi i} \int_{L} \frac{\Gamma(1-\sigma)}{\Gamma(1-\gamma \sigma)} t^{-\gamma \sigma}\left\{\mathcal{M} S_{\beta}\right\}(\sigma) d \sigma
$$

After some routine calculations using Fubini's theorem and the identities

$$
\int_{0}^{\infty} e^{-\lambda t} t^{z-1} d t=\Gamma(z) \lambda^{-z}, \operatorname{Re} z>0, \lambda>0 ; \frac{1}{2 \pi i} \int_{L} \Gamma(1-\sigma) u^{\sigma-1} d \sigma=e^{-u}, \quad u>0
$$

we find that the Laplace transform of $S(t)$ for $\lambda>0$ equals

$$
\int_{0}^{\infty} e^{-\lambda t} S(t) d t=\lambda^{\gamma-1} \int_{0}^{\infty} e^{-\lambda^{\gamma} t} S_{\beta}(t) d t=\lambda^{\alpha-1} R\left(\lambda^{\alpha}, A\right) .
$$

Then $S(t)=S_{\alpha}(t)$ follows from Theorem 2.9 .

In fact, (3.24) is an abstract version of a representation of the Mittag-Leffler function, given in [57], p.118. 


\subsection{The semigroup of subordination operators}

Besides the semigroups associated with the subordination of Volterra integral equations (see [64], p.116, [22]) in the special case of fractional evolution equations another interesting example of a semigroup appears. In this section we study its properties.

Let us look back at the subordination formula (3.3) and define a family of operators $G_{\gamma}$ for $\gamma \in(0,1]$ by

$$
\left(G_{\gamma} f\right)(t):= \begin{cases}\int_{0}^{\infty} \varphi_{t, \gamma}(s) f(s) d s, & \gamma \in(0,1) \\ f(t), & \gamma=1,\end{cases}
$$

where $f$ belongs to some appropriate function space $X$. Then (3.3) can be written in the form $S_{\alpha}=G_{\gamma} S_{\beta}$ with $\gamma=\alpha / \beta \in(0,1]$. For this reason we call $G_{\gamma}$ subordination operators. The following identity plays a crucial role:

$$
\int_{0}^{\infty} \varphi_{t, \gamma}(s) \varphi_{s, \delta}(\sigma) d s=\varphi_{t, \gamma \delta}(\sigma), \quad \gamma, \delta \in(0,1), \sigma>0 .
$$

Its proof is based on the equalities (3.9) and (3.10). Applying (3.28), $G_{\gamma}$ yields

$$
G_{\gamma} G_{\delta}=G_{\gamma \delta}, \quad \gamma, \delta \in(0,1],
$$

i.e. the family of operators $\left\{G_{e^{-\tau}}\right\}_{\tau \geq 0}$ has the semigroup property.

Assume that $X=L^{p}\left(\mathbb{R}_{+}, e^{-t} d t\right), p \geq 1$, consists of all measurable functions $f$ on $(0, \infty)$, such that

$$
\|f\|_{p}=\left(\int_{0}^{\infty} e^{-t}|f(t)|^{p} d t\right)^{1 / p}<\infty .
$$

Theorem 3.14 The family $\left\{G_{e^{-\tau}}\right\}_{\tau \geq 0}$ is a $C_{0}$-semigroup of contractions on $L^{p}\left(\mathbb{R}_{+}, e^{-t} d t\right)$.

Proof: First we prove that if $f \in L^{p}\left(\mathbb{R}_{+}, e^{-t} d t\right)$, then

$$
\left\|G_{\gamma} f\right\|_{p} \leq\|f\|_{p}, 0<\gamma \leq 1 .
$$

Applying Hölder's inequality and (1.32), we obtain

$$
\left|\int_{0}^{\infty} \varphi_{t, \gamma}(s) f(s) d s\right|^{p} \leq \int_{0}^{\infty} \varphi_{t, \gamma}(s)|f(s)|^{p} d s .
$$

Therefore, by use of Fubini's theorem and (3.10) with $\lambda=1$, we have

$$
\begin{aligned}
\left\|G_{\gamma} f\right\|_{p}^{p} & \leq \int_{0}^{\infty} e^{-t}\left(\int_{0}^{\infty} \varphi_{t, \gamma}(s)|f(s)|^{p} d s\right) d t \\
& =\int_{0}^{\infty}|f(s)|^{p}\left(\int_{0}^{\infty} e^{-t} \varphi_{t, \gamma}(s) d t\right) d s=\|f\|_{p}^{p} .
\end{aligned}
$$


Next we prove that if $f \in L^{p}\left(\mathbb{R}_{+}, e^{-t} d t\right)$, then $\left\|G_{\gamma} f-f\right\|_{p} \rightarrow 0$ as $\gamma \rightarrow 1^{-}$. Using the representations implied by $(3.27)$ and (1.32),

$$
\left(G_{\gamma} f\right)(t)=\int_{0}^{\infty} \Phi_{\gamma}(s) f\left(s t^{\gamma}\right) d s, \quad f(t)=\int_{0}^{\infty} \Phi_{\gamma}(s) f(t) d s,
$$

we obtain by Hölder's inequality

$$
\left\|G_{\gamma} f-f\right\|_{p}^{p} \leq \int_{0}^{\infty} e^{-t} \int_{0}^{\infty} \Phi_{\gamma}(s)\left|f\left(s t^{\gamma}\right)-f(t)\right|^{p} d s d t
$$

Suppose that $f \in C_{0}^{\infty}\left(\mathbb{R}_{+}\right)$, so that $f(t)$ is smooth on $(0, \infty)$ and has a compact support. Fix $\varepsilon>0$. By the uniform continuity of $f(t)$ on $(0, \infty)$ there exist $\delta, \delta_{0}>0$, such that $\left|f\left(s t^{\gamma}\right)-f(t)\right|^{p}<\varepsilon^{p} / 3$ whenever $1-\gamma<\delta_{0},|s-1|<\delta, t \in(0, \infty)$. Consider the integrals

$$
\begin{gathered}
I_{1}=\int_{0}^{1-\delta} \Phi_{\gamma}(s)\left|f\left(s t^{\gamma}\right)-f(t)\right|^{p} d s, \quad I_{2}=\int_{1-\delta}^{1+\delta} \Phi_{\gamma}(s)\left|f\left(s t^{\gamma}\right)-f(t)\right|^{p} d s, \\
I_{3}=\int_{1+\delta}^{\infty} \Phi_{\gamma}(s)\left|f\left(s t^{\gamma}\right)-f(t)\right|^{p} d s .
\end{gathered}
$$

For the second one we obtain immediately $I_{2}<\left(\varepsilon^{p} / 3\right) \int_{0}^{\infty} \Phi_{\gamma}(s) d s=\varepsilon^{p} / 3$. Since $f \in$ $C_{0}^{\infty}\left(\mathbb{R}_{+}\right)$, there exists a constant $M>0$, such that $\left|f\left(s t^{\gamma}\right)-f(t)\right|^{p}<M$ for all $s, t \in$ $(0, \infty), \gamma \in(0,1)$. Therefore $I_{1} \leq M \int_{0}^{1-\delta} \Phi_{\gamma}(s) d s, I_{3} \leq M \int_{1+\delta}^{\infty} \Phi_{\gamma}(s) d s$. To estimate $I_{1}$ and $I_{3}$ we use the integral representation of $\Phi_{\gamma}(s)$ given in (1.30). Applying Fubini's theorem, we have

$$
\int_{0}^{1-\delta} \Phi_{\gamma}(s) d s=\frac{1}{2 \pi i} \int_{\Gamma} \mu^{-1} e^{\mu}\left(1-e^{-(1-\delta) \mu^{\gamma}}\right) d \mu
$$

and similarly

$$
\int_{1+\delta}^{\infty} \Phi_{\gamma}(s) d s=\frac{1}{2 \pi i} \int_{\Gamma} \mu^{-1} e^{\mu-(1+\delta) \mu^{\gamma}} d \mu .
$$

Letting $\gamma \rightarrow 1^{-}$under the integral sign, which is justified by the dominated convergence theorem, and using the identity

$$
\frac{1}{2 \pi i} \int_{\omega-i \infty}^{\omega+i \infty} \mu^{-1} e^{c \mu} d \mu=\left\{\begin{array}{ll}
1, & c>0 \\
0, & c<0,
\end{array}, \omega>0\right.
$$

we obtain that both integrals tend to 0 . Hence, there exists $\delta_{1}$ such that $I_{1}<\varepsilon^{p} / 3, I_{3}<$ $\varepsilon^{p} / 3$, provided $1-\gamma<\delta_{1}$. Therefore, if $1-\gamma<\delta_{2}=\min \left\{\delta_{0}, \delta_{1}\right\}$, then $I_{1}+I_{2}+I_{3}<\varepsilon^{p}$ and, according to (3.32), $\left\|G_{\gamma} f-f\right\|_{p}<\varepsilon$. To prove this result for any $f \in L^{p}\left(\mathbb{R}_{+}, e^{-t} d t\right)$ we use the fact that $C_{0}^{\infty}\left(\mathbb{R}_{+}\right)$is dense in $L^{p}\left(\mathbb{R}_{+}, e^{-t} d t\right)$, which follows from its denseness in $L^{p}\left(\mathbb{R}_{+}\right)$, and (3.30). It remains to add the semigroup property (3.29) and the theorem is proved.

We now proceed to the determination of the infinitesimal generator $A_{G}$ of the semigroup $\left\{G_{e^{-\tau}}\right\}_{\tau \geq 0}$. For the resolvent operator we obtain the following representation

$$
\left(R\left(\lambda, A_{G}\right) f\right)(t)=\int_{0}^{\infty} e^{-\lambda \tau}\left(G_{e^{-\tau}} f\right)(t) d \tau=\int_{0}^{\infty} E(t, s, \lambda) f(s) d s,
$$


where

$$
E(t, s, \lambda):=\int_{0}^{1} \sigma^{\lambda-1} \varphi_{t, \sigma}(s) d \sigma, t, s \geq 0 .
$$

In the particular case $\lambda=1$, inserting (3.9) in the above identity, we have

$$
E(t, s, 1)=s^{-1}\left(e^{-s} \nu(t)-\nu(t-s)\right), t, s \geq 0,
$$

where

$$
\nu(t):= \begin{cases}\int_{0}^{\infty} \frac{t^{u}}{\Gamma(u+1)} d u, & t \geq 0 \\ 0, & t<0 .\end{cases}
$$

For the properties of $\nu(t)$ see [32], Vol. 3, Chapter 18. Next we give an explicit expression for the infinitesimal generator. Denote for $m \in \mathbb{N}$

$$
W_{0}^{m, p}\left(\mathbb{R}_{+}, e^{-t} d t\right):=\left\{f \mid \exists \varphi \in L^{p}\left(\mathbb{R}_{+}, e^{-t} d t\right): f(t)=\left(J_{t}^{m} \varphi\right)(t)\right\}
$$

Theorem 3.15 Consider the semigroup $\left\{G_{e^{-\tau}}\right\}_{\tau \geq 0}$ on $L^{p}\left(\mathbb{R}_{+}, e^{-t} d t\right)$. Its infinitesimal generator $A_{G}$ is defined by

$$
\begin{gathered}
D\left(A_{G}\right)=\left\{f \in L^{p}\left(\mathbb{R}_{+}, e^{-t} d t\right) \mid J_{t}^{1}(l * f)-l *(t f) \in W_{0}^{2, p}\left(\mathbb{R}_{+}, e^{-t} d t\right)\right\}, \\
\left(A_{G} f\right)(t)=\frac{d}{d t}(l * f)-\frac{d^{2}}{d t^{2}}(l *(t f)),
\end{gathered}
$$

where $l(t):=\log C t, C$-Euler's constant.

Proof: Suppose that $f \in D\left(A_{G}\right)$. Then $\left(G_{e^{-\tau}} A_{G} f\right)(t)=\left(\frac{\partial}{\partial \tau} G_{e^{-\tau}} f\right)(t)$. Applying the integration operator $J_{t}^{3}$ to both sides, we obtain after some routine calculations, using (3.27), (3.9) and Fubini's theorem

$$
J_{t}^{3}\left(G_{\gamma} A_{G} f\right)(t)=\frac{-\gamma}{2 \pi i} \int_{\Gamma_{1}} \mu^{\gamma-4} \log \mu\left(F\left(\mu^{\gamma}\right)+\mu^{\gamma} F^{\prime}\left(\mu^{\gamma}\right)\right) e^{\mu t} d \mu,
$$

where $\gamma=e^{-\tau}, \Gamma_{1}=(\omega-i \infty, \omega+i \infty), \omega>1$, and $F(\lambda)=\int_{0}^{\infty} e^{-\lambda t} f(t) d t$. In this identity we let $\gamma \rightarrow 1^{-}$, applying on the left the strong continuity of $G_{e^{-\tau}}$ and on the right the dominated convergence theorem. It follows

$$
J_{t}^{3}\left(A_{G} f\right)(t)=-\frac{1}{2 \pi i} \int_{\Gamma_{1}} \mu^{-3} \log \mu\left(F(\mu)+\mu F^{\prime}(\mu)\right) e^{\mu t} d \mu .
$$

If we set $\phi(t):=l_{2} * f, \psi(t):=l_{1} *(t f)$, where $l_{i}=J_{t}^{i} l, i=1,2$, then by the properties of the Laplace transform

$$
\Phi(\mu):=\int_{0}^{\infty} e^{-\mu t} \phi(t) d t=-\frac{\log \mu}{\mu^{3}} F(\mu), \Psi(\mu):=\int_{0}^{\infty} e^{-\mu t} \psi(t) d t=\frac{\log \mu}{\mu^{2}} F^{\prime}(\mu) .
$$

Moreover, the integrals representing $\Phi(\mu)$ and $\Psi(\mu)$ are absolutely convergent. Indeed, fix $\omega=\operatorname{Re} \mu>1$, then by Young's inequality

$$
\int_{0}^{\infty} e^{-\omega t}|\phi(t)| d t \leq \int_{0}^{\infty} e^{-\omega t}\left|l_{2}(t)\right| d t \int_{0}^{\infty} e^{-\omega t}|f(t)| d t=C \int_{0}^{\infty} e^{-\omega t}|f(t)| d t .
$$


Now, by means of Hölder's inequality when $p>1$ (and for $p=1$ because $\omega>1$ ), we have the estimate

$$
\int_{0}^{\infty} e^{-\omega t}|f(t)| d t \leq K_{p}\left(\int_{0}^{\infty} e^{-t}|f(t)|^{p} d t\right)^{1 / p}=K_{p}\|f\|_{p}<\infty
$$

where $K_{1}=1 ; K_{p}=\left(\frac{\omega p-1}{p-1}\right)^{\frac{1-p}{p}}, p>1$. The absolute convergence of the second integral follows in the same way. Moreover, $\phi(t)$ and $\psi(t)$ are absolutely continuous functions, therefore we can apply the theorem for the inverse Laplace transform [73], Theorem 7.3, p. 66, to $(3.36)$, which implies $J_{t}^{3}\left(A_{G} f\right)(t)=-\phi(t)+\psi(t)$, or

$$
J_{t}^{2}\left(A_{G} f\right)(t)=-J_{t}^{1}(l * f)+l *(t f) .
$$

Since $A_{G} f \in L^{p}\left(\mathbb{R}_{+}, e^{-t} d t\right)$, it follows that

$$
-J_{t}^{1}(l * f)+l *(t f) \in W_{0}^{2, p}\left(\mathbb{R}_{+}, e^{-t} d t\right)
$$

and differentiation of (3.37) with respect to $t$ implies (3.35).

Conversely, suppose (3.38). Then an elementary calculation shows that

$$
\int_{\alpha}^{\beta} G_{e^{-\tau}}\left(\frac{d}{d t}(l * f)-\frac{d^{2}}{d t^{2}}(l *(t f))\right) d \tau=G_{e^{-\beta}} f-G_{e^{-\alpha}} f, 0<\alpha<\beta<\infty .
$$

Here we let $\alpha \rightarrow 0^{+}$. Since $G_{e^{-\tau}}$ is of class $C_{0}$ the right member tends to $G_{e^{-\beta}} f-f$. Then [46], Theorem 10.5.2, applies and shows that $f \in D\left(A_{G}\right)$ and that (3.35) holds. 


\section{Chapter 4}

\section{Strict $L^{p}$ solvability}

We study the strict $L^{p}$ solvability of the linear autonomous nonhomogeneous problem for the fractional differential equation with Riemann-Liouville fractional derivative of order $\alpha \in(0,2)$. Our analysis combines two different approaches. First we prove maximal $L^{p}$ regularity of the problem with zero initial conditions using the method of sums of operators. We apply both classical theorems and some very recent results involving the notion of $\mathcal{R}$-boundedness. Concerning the problem with zero forcing function, we use the resolvent approach and prove strict $L^{p}$ solvability provided the initial data belongs to some real interpolation spaces.

\subsection{The notion of maximal $L^{p}$ regularity}

The notion of maximal $L^{p}$ regularity plays an important role in the functional analytic approach to parabolic partial differential equations. Many initial and boundary value problems can be reduced to an abstract Cauchy problem of the form

$$
u^{\prime}(t)+A u(t)=f(t), t \in I, u(0)=0,
$$

where $I=(0, T), T>0,-A$ generates a bounded analytic semigroup on a Banach space $X$ and $f$ and $u$ are $X$-valued functions on $I$. It is well known that (4.1) has a strong solution for all locally Bochner integrable $f$, but in many applications we need that $u^{\prime}$ has the same "smoothness" as $f$, which is not always the case. In particular, one says that problem (4.1) has maximal $\boldsymbol{L}^{\boldsymbol{p}}$ regularity on $I$ if for every $f \in L^{p}(I ; X)$ there exists one and only one $u \in L^{p}(I ; D(A)) \cap W^{1, p}(I ; X)$ satisfying (4.1). From the closed graph theorem it follows easily that if there is $L^{p}$ regularity then there exists $C>0$ such that

$$
\|u\|_{L^{p}}+\left\|u^{\prime}\right\|_{L^{p}}+\|A u\|_{L^{p}} \leq C\|f\|_{L^{p}}
$$

The theory of strongly continuous semigroups could suggest that it is more natural to study the continuous regularity for (4.1), i.e. the existence and uniqueness of a solution $u \in C(I ; D(A)) \cap C^{1}(I ; X)$ for any continuous $f$. But Baillon [3] proved that if there is 
continuous regularity for an unbounded operator $A$ that generates a $C_{0}$ semigroup, then the space $X$ must contain a subspace isomorphic to $c_{0}$, the space of sequences converging to 0 . This fact implies that $X$ cannot be reflexive. On the other hand there are good results of $L^{p}$ regularity in some reflexive spaces.

There is a rich literature on sufficient conditions for maximal $L^{p}$ regularity (see for a survey [27]), which implies that for most classical differential operators that may be of interest, there is maximal $L^{p}$ regularity of problem (4.1). Quite recently, necessary and sufficient conditions for maximal $L^{p}$ regularity was obtained in terms of $\mathcal{R}$-boundedness. For completeness, we present the definition of this notion ( see e.g. [20] ).

Definition 4.1 A family $\tau \subset B(X)$ is called $\mathcal{R}$-bounded (randomized bounded), if there exists a constant $M>0$, such that

$$
\sum_{\varepsilon \in\{-1,1\}^{N}}\left\|\sum_{j=0}^{N} \varepsilon_{j} T_{j} x_{j}\right\|^{2} \leq M^{2} \sum_{\varepsilon \in\{-1,1\}^{N}}\left\|\sum_{j=0}^{N} \varepsilon_{j} x_{j}\right\|^{2}
$$

holds for all $\left\{T_{j}\right\}_{j=1}^{N} \subset \tau$, all $\left\{x_{j}\right\}_{j=1}^{N} \subset X$, and all $N \in \mathbb{N}$. The smallest constant $M$ such that (4.3) holds is called the $\mathcal{R}$-bound of $\tau$ and is denoted by $\mathcal{R}(\tau)$.

This definition can be seen as a straightening of the concept of uniform boundedness of the family $\tau$.

The following theorem is due independently to N. Kalton and L. Weis. For a proof see e.g. [72].

Theorem 4.2 Let $-A$ generates a bounded analytic semigroup on an $\mathcal{H} \mathcal{T}$ space $X$. Then problem (4.1) has maximal $L^{p}$ regularity if and only if the set $\left\{\lambda(\lambda+A)^{-1} \mid \lambda \in i \mathbb{R}, \lambda \neq 0\right\}$ is $\mathcal{R}$-bounded.

In Hilbert spaces the uniform boundedness of this set already implies maximal $L^{p}$ regularity, but only in Hilbert space: recently Kalton and Lancien [47] essentially proved that if for every negative generator $A$ of a bounded analytic semigroup on a Banach space $X$ problem (4.1) has maximal $L^{p}$ regularity, then $X$ is isomorphic to a Hilbert space. It appears that the additional assumption which we need in more general Banach spaces is namely the $\mathcal{R}$-boundedness of the set.

Maximal $L^{p}$ regularity is an important tool in treating evolution equations more complex than the basic Cauchy problem (4.1), such as second order equations, Volterra equations, nonautonomous and quasilinear equations. In this thesis we apply maximal $L^{p}$ regularity to study fractional order equations: both autonomous and nonautonomous. 


\subsection{Maximal $L^{p}$ regularity for fractional order equa- tions}

Let $A$ be a linear closed densely defined operator on a Banach space $X$. Let $I=(0, T)$ for some $T>0$. Consider the Cauchy problem for the fractional differential equation with Riemann-Liouville fractional derivative of order $\alpha \in(\mathbf{0 , 2})$

$$
D_{t}^{\alpha} u(t)+A u(t)=f(t), \text { a.a. } t \in I,
$$

with an initial condition $\left(g_{1-\alpha} * u\right)(0)=0$ when $\alpha \in(0,1)$ and two initial conditions $\left(g_{2-\alpha} * u\right)(0)=0$ and $\left(g_{2-\alpha} * u\right)^{\prime}(0)=0$ when $\alpha \in(1,2)$.

Let $R_{0}^{\alpha, p}(I ; X)$ be the domain of the operator of fractional differentiation, defined by (1.35). All other notations in this section are also defined in Chapter 1.

Definition 4.3 We say that there is maximal $\boldsymbol{L}^{\boldsymbol{p}}$ regularity of (4.4), on $I$, in $X$, if for every $f \in L^{p}(I ; X)$ there exists one and only one $u \in L^{p}(I ; D(A)) \cap R_{0}^{\alpha, p}(I ; X)$ satisfying (4.4).

It follows from the closed graph theorem that if there is $L^{p}$ regularity of (4.4) then there exists $C>0$ such that

$$
\|u\|_{L^{p}}+\left\|D_{t}^{\alpha} u\right\|_{L^{p}}+\|A u\|_{L^{p}} \leq C\|f\|_{L^{p}}
$$

Following Da Prato and Grisvard [23], we rewrite the equation (4.4) for $X$-valued functions $u$ and $f$ as an operator equation in $\mathcal{X}=L^{p}(I ; X)$. To this end we define the linear closed operator $\mathcal{A}$ on $\mathcal{X}$ by

$$
D(\mathcal{A})=L^{p}(I ; D(A)) ;(\mathcal{A} u)(t)=A u(t), u \in D(\mathcal{A}),
$$

and take $\mathcal{B}=\mathcal{L}_{\alpha}$, where $\mathcal{L}_{\alpha}$ is defined by (1.37). Then rewrite equation (4.4) as

$$
\mathcal{A} u+\mathcal{B} u=f, \quad f \in \mathcal{X} .
$$

More than 20 years ago Da Prato and Grisvard [23] found sufficient conditions for maximal regularity of (4.7) in real interpolation spaces. Later, Dore and Venni [28] solved this problem in the case of $\mathcal{H} \mathcal{T}$ space. Here we present these theorems, reformulated suitably for our application (see [14], Theorem 4, and [1], Theorem 4.9.7 and Corollary 4.9.8).

Theorem 4.4 ( Da Prato-Grisvard) Let $\mathcal{X}$ be a complex Banach space and $\mathcal{A}$ and $\mathcal{B}$ be nonnegative operators in $\mathcal{X}$ with spectral angles $\omega_{\mathcal{A}}$ and $\omega_{\mathcal{B}}$, respectively, such that

$$
\omega_{\mathcal{A}}+\omega_{\mathcal{B}}<\pi
$$

Let moreover $\mathcal{A}$ and $\mathcal{B}$ be resolvent commuting and satisfy $0 \in \rho(A) \cup \rho(B)$. If $\mathcal{Y}$ is one of the spaces $D_{\mathcal{A}}(\delta, q)$ or $D_{\mathcal{B}}(\delta, q)$, where $\delta \in(0,1)$ and $q \in[1, \infty]$, then for any $f \in \mathcal{Y}$ there is a unique $u \in D(\mathcal{A}) \cap D(\mathcal{B})$ such that $\mathcal{A} u+\mathcal{B} u=f$. Moreover, $\mathcal{A} u$ and $\mathcal{B} u \in \mathcal{Y}$ and

$$
\|u\|_{\mathcal{Y}}+\|\mathcal{A} u\|_{\mathcal{Y}}+\|\mathcal{B} u\|_{\mathcal{Y}} \leq C\|f\|_{\mathcal{Y}}
$$

where the constant $C$ depends on $\mathcal{X}, \delta, q, \omega_{\mathcal{A}}, \omega_{\mathcal{B}}, K_{\mathcal{A}}(\pi-\theta)$ and $K_{\mathcal{B}}(\theta)$ for some $\theta \in\left(\omega_{\mathcal{A}}, \pi-\omega_{\mathcal{B}}\right)$, but not on the individual operators $\mathcal{A}$ and $\mathcal{B}$. 
Theorem 4.5 ( Dore-Venni ) Let $\mathcal{X}$ be an $\mathcal{H} \mathcal{T}$ space. Assume

$$
\mathcal{A} \in \mathcal{P}_{K}(\mathcal{X}) \cap \mathcal{B I} \mathcal{P}\left(\mathcal{X} ; M, \varphi_{\mathcal{A}}\right), \mathcal{B} \in \mathcal{P}_{K}(\mathcal{X}) \cap \mathcal{B I} \mathcal{P}\left(\mathcal{X} ; M, \varphi_{\mathcal{B}}\right)
$$

with

$$
\varphi_{\mathcal{A}}+\varphi_{\mathcal{B}}<\pi
$$

and let $\mathcal{A}$ and $\mathcal{B}$ be resolvent commuting. Then for any $f \in \mathcal{X}$ there is a unique $u \in$ $D(\mathcal{A}) \cap D(\mathcal{B})$ such that $\mathcal{A} u+\mathcal{B} u=f$. Moreover,

$$
\|u\|_{\mathcal{X}}+\|\mathcal{A} u\|_{\mathcal{X}}+\|\mathcal{B} u\|_{\mathcal{X}} \leq C\|f\|_{\mathcal{X}}
$$

holds, where the constant $C$ depends on $\mathcal{X}, K, M, \varphi_{\mathcal{A}}$ and $\varphi_{\mathcal{B}}$, but not on the individual operators $\mathcal{A}$ and $\mathcal{B}$.

Next we apply Theorems 4.4 and 4.5 to our concrete problem (4.4).

Corollary 4.6 Let $\alpha \in(0,2), 1<p<\infty, \delta \in(0,1)$. Let $A$ be a positive operator in a Banach space $X$ with spectral angle satisfying

$$
\omega_{A}<\pi(1-\alpha / 2)
$$

(a) If $Y=(X, D(A))_{\delta, p}$, then (4.4) has maximal $L^{p}$ regularity on $I$ in the space $Y$. More precisely, the following estimate for the solution $u$ holds:

$$
\|u\|_{L^{p}(I ; Y)}+\left\|D_{t}^{\alpha} u\right\|_{L^{p}(I ; Y)}+\|A u\|_{L^{p}(I ; Y)} \leq C_{1}\|f\|_{L^{p}(I ; Y)}
$$

(b) Let $\alpha \delta-1 / p \notin \mathbb{N}_{0}$. For any $f \in W_{0}^{\alpha \delta, p}(I ; X)$ there exists a unique solution $u$ of (4.4). Moreover, $D_{t}^{\alpha} u, A u \in W_{0}^{\alpha \delta, p}(I ; X)$ and

$$
\|u\|_{W^{\alpha \delta, p}(I ; X)}+\left\|D_{t}^{\alpha} u\right\|_{W^{\alpha \delta, p}(I ; X)}+\|A u\|_{W^{\alpha \delta, p}(I ; X)} \leq C_{2}\|f\|_{W^{\alpha \delta, p}(I ; X)} .
$$

The constants $C_{1}$ and $C_{2}$ depend on $\alpha, p, \delta, \omega_{A}$ and $K_{A}(\theta)$ for some $\theta \in\left(\alpha \pi / 2, \pi-\omega_{A}\right)$, but not on $T$ and on the individual operator $A$.

Proof: We apply first Theorem 4.4 to the problem on $L^{p}(\mathbb{R} ; X)$ in order to obtain a constant, which does not depend on $T$. Let $f \in L^{p}(I ; Y)$ and define the function

$$
f_{0}= \begin{cases}f, & t \in[0, T] \\ 0, & t \notin[0, T]\end{cases}
$$

Then $f_{0} \in L^{p}(\mathbb{R} ; Y)$. We extend the definition of the fractional derivative to functions on $L^{p}(\mathbb{R})$ as follows. Let $u \in C_{0}^{\infty}(\mathbb{R})$, which is dense in $L^{p}(\mathbb{R})$. Define

$$
L_{\alpha} u:=D_{t}^{m}\left(g_{m-\alpha} * u\right), \quad m=\lceil\alpha\rceil,
$$

where $*$ is the convolution on $\mathbb{R}$. This operator is nonnegative, therefore closable, and we take its closure in $L^{p}(\mathbb{R})$ as definition of fractional derivative on $L^{p}(\mathbb{R})$. We use the same 
notation $L_{\alpha}$. Let $\mathcal{L}_{\alpha}, \mathcal{A}$ denote the extensions of $L_{\alpha}, A$ to $L^{p}(\mathbb{R} ; X)$. For the Fourier transform of $\mathcal{L}_{\alpha} u$ we have

$$
\widetilde{\mathcal{L}_{\alpha} u}(\rho)=(i \rho)^{\alpha} \tilde{u}(\rho), \quad \rho \in \mathbb{R} \backslash\{0\}, \tilde{f} \in C_{0}^{\infty}(\mathbb{R} \backslash\{0\} ; X) .
$$

Therefore, according to [64], Theorem 8.6, $\mathcal{L}_{\alpha}$ is a sectorial operator in $\mathcal{X}=L^{p}(\mathbb{R} ; X)$ with spectral angle $\omega_{\mathcal{L}_{\alpha}}=\alpha \pi / 2$. It is immediate that $\mathcal{A}$ is a positive operator on $\mathcal{X}$ with spectral angle $\omega_{\mathcal{A}}=\omega_{\mathcal{A}}$. Consider the problem on $\mathbb{R}$ :

$$
\mathcal{L}_{\alpha} u+\mathcal{A} u=f_{0} .
$$

By (4.9) operators $\mathcal{A}$ and $\mathcal{L}_{\alpha}$ satisfy conditions of Theorem 4.4. If we take $\mathcal{Y}=(\mathcal{X}, D(\mathcal{A}))_{\delta, p}$, we obtain by [70], Theorem 1.18.4,

$$
\mathcal{Y}=\left(L^{p}(\mathbb{R} ; X), L^{p}(\mathbb{R} ; D(A))\right)_{\delta, p}=L^{p}\left(\mathbb{R} ;(X, D(A))_{\delta, p}\right)=L^{p}(\mathbb{R} ; Y),
$$

and Theorem 4.4 implies (a) on $L^{p}(\mathbb{R} ; Y)$. Now turn back to our equation (4.4) on $L^{p}(I ; Y)$. Denote by $u_{0}$ the solution of (4.13). Because of the causality of the equation, $u_{0}=0$ for $t<0$. This easily implies that $u_{0}$ satisfies the initial conditions of problem (4.4). Therefore, the restriction $u(t)$ of $u_{0}(t)$ to $I$ will be a solution of (4.4), satisfying (4.10), and (a) is proved. The claim concerning the constant follows from the corresponding claim in Theorem 4.4.

Applying the same argument we prove (b) taking $\mathcal{Y}=\left(\mathcal{X}, D\left(\mathcal{L}_{\alpha}\right)\right)_{\delta, p}$, which by (1.41) is equivalent to $\mathcal{Y}=W_{0}^{\alpha \delta, p}$ for $\alpha \delta-1 / p \notin \mathbb{N}_{0}$.

Corollary 4.7 Let $\alpha \in(0,2), A$ be a positive operator in an $\mathcal{H} \mathcal{T}$ space $X$ satisfying $A \in \mathcal{P}_{K}(X) \cap \mathcal{B I P}\left(X ; M, \varphi_{A}\right)$ with

$$
\varphi_{A}<\pi(1-\alpha / 2)
$$

Then (4.4) has maximal $L^{p}$ regularity on $I$ in $X$. More precisely,

$$
\|u\|_{L^{p}(I ; X)}+\|u\|_{H^{\alpha, p}(I ; X)}+\|A u\|_{L^{p}(I ; X)} \leq C\|f\|_{L^{p}(I ; X)},
$$

where $C$ depends on $\alpha, p, K, M, \varphi_{A}, T$, but not on the individual operator $A$.

Proof: First note that $\mathcal{X}=L^{p}(I ; X)$ is an $\mathcal{H} \mathcal{T}$ space, because $X$ is and $1<p<\infty$. Since $A \in \mathcal{P}_{K}(X) \cap \mathcal{B I} \mathcal{P}\left(X ; M, \varphi_{A}\right)$, for the extension $\mathcal{A}$ of $A$ to $\mathcal{X}$ we have $\mathcal{A} \in \mathcal{P}_{K}(\mathcal{X}) \cap$ $\mathcal{B I P}\left(\mathcal{X} ; M, \varphi_{A}\right)$. Then Lemma 1.8, (d), and (4.14) imply that the conditions of Theorem 4.5 are satisfied and we obtain the desired result. Because $X$ is of class $\mathcal{H T}, R_{0}^{\alpha, p}=H_{0}^{\alpha, p}$ for $\alpha-1 / p \neq 0,1$, and therefore $\left\|D_{t}^{\alpha} u\right\|_{L^{p}(I ; X)}=\|u\|_{H^{\alpha, p}(I ; X)}$.

If we want to prove that the constant $C$ does not depend on $T$, we have to apply a generalization of Theorem 4.5 to sectorial operators [65] and work first on $L^{p}(\mathbb{R} ; X)$ as in the proof of the previous corollary. We skip this argument, because in what follows we present a stronger result. 
Next we formulate weaker conditions on $A$, sufficient for maximal $L^{p}$ regularity of (4.4). This is possible applying the following very recent generalization of the Michlin multiplier theorem due to Weis [72], Clément and Prüss [22].

Let $\mathcal{S}(\mathbb{R} ; X)$ be the space of Schwartz of smooth rapidly decreasing $X$-valued functions and $\mathcal{S}^{\prime}(\mathbb{R} ; X)$ be the space of $X$-valued distributions. Let $m: \mathbb{R} \backslash\{0\} \rightarrow \mathcal{B}(X)$ be differentiable and define for $f \in \mathcal{S}(\mathbb{R} ; X)$ the function $\mathbf{M} f \in \mathcal{S}^{\prime}(\mathbb{R} ; X)$ by

$$
\widetilde{\mathbf{M}} f(\rho):=m(\rho) \tilde{f}(\rho), \rho \in \mathbb{R} \backslash\{0\},
$$

where $\tilde{f}$ denotes the Fourier transform of $f$.

Theorem 4.8 Let $X$ be an $\mathcal{H} \mathcal{T}$ space, $1<p<\infty$, and let $m \in C^{1}(\mathbb{R} \backslash\{0\} ; \mathcal{B}(X))$ be such that the following two conditions are satisfied

(i) $\mathcal{R}(\{m(\rho) \mid \rho \in \mathbb{R} \backslash\{0\}\})=: k_{0}<\infty$,

(ii) $\mathcal{R}\left(\left\{\rho m^{\prime}(\rho) \mid \rho \in \mathbb{R} \backslash\{0\}\right\}\right)=: k_{1}<\infty$.

Then the operator $\mathbf{M}$, defined by (4.16), extends to a bounded operator on $L^{p}(\mathbb{R} ; X)$. Its bound depends only on $X, p, k_{0}$ and $k_{1}$.

For a proof, we refer to [22], Theorem 1. The statement about the bound is implicitly given in this proof.

It was proven ([22], Proposition 1.) that the $\mathcal{R}$-boundedness condition (i) is also necessary for $\mathbf{M}$ to be extended to a bounded operator on $L^{p}(\mathbb{R} ; X)$.

Let just as in the proof of Corollary $4.6 \mathcal{L}_{\alpha}$ be the fractional derivative in $L^{p}(\mathbb{R} ; X), \mathcal{A}$ be the extension of $A$ to $L^{p}(\mathbb{R} ; X)$ and define $f_{0}$ as in (4.11). Consider the corresponding problem on $\mathbb{R}$ :

$$
\mathcal{L}_{\alpha} u+\mathcal{A} u=f_{0} .
$$

Applying Fourier transform, we obtain

$$
\widetilde{u}(\rho)=\left((i \rho)^{\alpha} I+A\right)^{-1} \widetilde{f}_{0}(\rho), \quad \rho \in \mathbb{R} \backslash\{0\} .
$$

Therefore we have the estimate

$$
\left\|\mathcal{L}_{\alpha} u\right\|_{L^{p}(\mathbb{R} ; X)}+\|\mathcal{A} u\|_{L^{p}(\mathbb{R} ; X)} \leq M\left\|f_{0}\right\|_{L^{p}(\mathbb{R} ; X)}
$$

iff the operator $\mathbf{M}$ defined by

$$
\widetilde{\mathbf{M} f}(\rho):=A\left((i \rho)^{\alpha} I+A\right)^{-1} \tilde{f}(\rho), \rho \in \mathbb{R} \backslash\{0\},
$$

is a bounded operator on $L^{p}(\mathbb{R} ; X)$. So, set $m(\rho):=A\left((i \rho)^{\alpha} I+A\right)^{-1}$. Suppose that $A$ is nonnegative with spectral angle, satisfying (4.9). Then $m(\rho)$ and $\rho m^{\prime}(\rho)$ for $\rho \in \mathbb{R} \backslash\{0\}$ are bounded operator valued functions, that is $m \in C^{1}(\mathbb{R} \backslash\{0\} ; \mathcal{B}(X))$. Hence, to obtain the boundedness of $\mathbf{M}$ on $L^{p}(\mathbb{R} ; X), 1<p<\infty$, we have to check conditions (i) and (ii) 
for $m(\rho)$ of Theorem 4.8. Since we have the representation $\rho m^{\prime}(\rho)=-\alpha(I-m(\rho)) m(\rho)$ and since the product of two $\mathcal{R}$-bounded families is again $\mathcal{R}$-bounded, it follows that condition (i) of Theorem 4.8 for our concrete function $m(\rho)$ implies condition (ii). That is, the maximal regularity estimate (4.18) holds iff the family of operators

$$
\left\{\lambda^{\alpha}\left(\lambda^{\alpha} I+A\right)^{-1} \mid \lambda \in i \mathbb{R}, \quad \lambda \neq 0\right\}
$$

is $\mathcal{R}$-bounded.

Definition 4.9 A sectorial operator $A$ on $X$ is called $\mathcal{R}$-sectorial if

$$
\mathcal{R}_{A}(0):=\mathcal{R}\left\{t(t I+A)^{-1} \mid t>0\right\}<\infty .
$$

The $\mathcal{R}$-angle $\omega_{A}^{R}$ of $A$ is defined by means of

$$
\omega_{A}^{R}:=\inf \left\{\theta \in(0, \pi) \mid \mathcal{R}_{A}(\pi-\theta)<\infty\right\},
$$

where

$$
\mathcal{R}_{A}(\theta):=\mathcal{R}\left(\left\{\lambda(\lambda I+A)^{-1} \mid \lambda \in \bar{\Sigma}_{\theta} \backslash\{0\}\right\}\right) .
$$

It is immediate that $\omega_{A}^{R} \geq \omega_{A}$. It has been shown by Weis [72] that $\mathcal{R}$-sectorial operators behave well under perturbations, like the class of sectorial operators.

We prove now the following result for the problem on $I$.

Proposition 4.10 Let $\alpha \in(0,2), 1<p<\infty, X$ be a Banach space of class $\mathcal{H} \mathcal{T}, A$ be an $\mathcal{R}$-sectorial operator on $X$ with $0 \in \rho(A)$ and $\mathcal{R}$-angle, satisfying

$$
\omega_{A}^{R}<\pi(1-\alpha / 2)
$$

Then problem (4.4) has maximal $L^{p}$ regularity and the following estimate holds

$$
\|u\|_{L^{p}(I ; X)}+\|u\|_{H^{\alpha, p}(I ; X)}+\|A u\|_{L^{p}(I ; X)} \leq C\|f\|_{L^{p}(I ; X)},
$$

where the constant $C$ depends on $X, p, \alpha, \mathcal{R}_{A}(\alpha \pi / 2)$, but not on $T$ and on the individual operator $A$.

Proof: Condition (4.20) implies that the family of operators $(4.19)$ is $\mathcal{R}$-bounded. Therefore, according to Theorem 4.8, for any $u \in D\left(\mathcal{L}_{\alpha}\right) \cap D(\mathcal{A})$ the estimate (4.18) holds. Consider equation $(4.17)$ on $L^{p}(\mathbb{R} ; X)$. Since $\mathcal{L}_{\alpha}$ and $\mathcal{A}$ are resolvent commuting, $0 \in \rho(\mathcal{A})$ and $\omega_{\mathcal{L}_{\alpha}}+\omega_{\mathcal{A}}<\pi$ (see the proof of Corollary 4.6 ), the pair of operators $\left(\mathcal{L}_{\alpha}, \mathcal{A}\right)$ is an admissible pair in $L^{p}(\mathbb{R} ; X)$ in the sense of [13], Definition 3.2, and, according to Theorem 3.3 of the same reference, the equation

$$
u+\mathcal{L}_{\alpha} \mathcal{A}^{-1} u=\mathcal{A}^{-1} f_{0}, f_{0} \in L^{p}(\mathbb{R} ; X),
$$

has a solution $u$ satisfying

$$
\|u\|_{L^{p}(\mathbb{R} ; X)} \leq C_{\mathcal{A}, \theta} K_{\mathcal{L}_{\alpha}}(\theta)\left\|f_{0}\right\|_{L^{p}(\mathbb{R} ; X)}
$$


for some $\theta \in\left(\alpha \pi / 2, \pi-\omega_{\mathcal{A}}\right)$, where $C_{\mathcal{A}, \theta}$ depends only on $\mathcal{A}$ and $\theta$. This solution is called mild solution of (4.17) and it becomes its strict solution if $u \in D\left(\mathcal{L}_{\alpha}\right)$ or $u \in D(\mathcal{A})$.

Combining (4.18) and (4.23), we obtain the full estimate for the mild solution

$$
\|u\|_{L^{p}(\mathbb{R} ; X)}+\|\mathcal{A} u\|_{L^{p}(\mathbb{R} ; X)}+\left\|\mathcal{L}_{\alpha} u\right\|_{L^{p}(\mathbb{R} ; X)} \leq C\left\|\mathcal{L}_{\alpha} u+\mathcal{A} u\right\|_{L^{p}(\mathbb{R} ; X)} .
$$

Using this estimate, we shall prove that (4.17) has a strict solution for any $f_{0} \in L^{p}(\mathbb{R} ; X)$. We know ([13], p.22, Remark), that if $f_{0} \in D\left(\mathcal{L}_{\alpha}\right)$ then the solution $u$ of $(4.22)$ belongs to $D\left(\mathcal{L}_{\alpha}\right)$ and so, it is a strict solution of $(4.17)$. Take a sequence $f_{n} \in W^{2, p}(\mathbb{R} ; X)$ such that $f_{n} \rightarrow f_{0}$ in $L^{p}$. Since $f_{n} \in W^{2, p} \subset D\left(\mathcal{L}_{\alpha}\right)$, then the equation (4.17) with right-hand side $f_{n}$ has a strict solution, denoted by $u_{n}$. Applying estimate (4.24) to the difference of two such equations, we obtain in $L^{p}(\mathbb{R} ; X)$ :

$$
\left\|u_{n}-u_{m}\right\|+\left\|\mathcal{A}\left(u_{n}-u_{m}\right)\right\|+\left\|\mathcal{L}_{\alpha}\left(u_{n}-u_{m}\right)\right\| \leq C\left\|f_{n}-f_{m}\right\| .
$$

Hence $u_{n}, \mathcal{L}_{\alpha} u_{n}, \mathcal{A} u_{n}$, are Cauchy sequences. The closedness of the operators $\mathcal{L}_{\alpha}$ and $\mathcal{A}$ implies that there exists $u \in D\left(\mathcal{L}_{\alpha}\right) \cap D(\mathcal{A})$ such that $u_{n} \rightarrow u, \mathcal{L}_{\alpha} u_{n} \rightarrow \mathcal{L}_{\alpha} u, \mathcal{A} u_{n} \rightarrow \mathcal{A} u$, in $L^{p}$. Therefore $u$ is a strict solution of (4.17).

Turn back to our equation (4.4) on $L^{p}(I ; X)$. Its solution is obtained as a restriction of the solution of (4.17) to [0,T]. Estimate (4.21) will follow from (4.24).

It is proven in [22], Theorem 4, that if $X$ is of class $\mathcal{H T}$ and $A \in \mathcal{B I P}\left(X ; \theta_{A}\right)$ then $A$ is $\mathcal{R}$-sectorial and $\omega_{A}^{R} \leq \theta_{A}$. Therefore Corollary 4.7 can be obtained from Proposition 4.10.

In fact, under the conditions of Proposition 4.10 we have even more: not only maximal $L^{p}$ regularity of (4.4), but also $\lambda$-regularity.

Definition 4.11 Let $X$ be a Banach space. The pair of closed operators $(\mathcal{A}, \mathcal{B})$ is called $\lambda$-regular in $X$ if for any $f \in X, \lambda>0$ the problem

$$
\lambda \mathcal{A} u+\mathcal{B} u=f
$$

has a unique solution $u \in D(\mathcal{A}) \cap D(\mathcal{B})$ and the following inequality holds

$$
\|\lambda \mathcal{A} u\|+\|\mathcal{B} u\| \leq M\|\lambda \mathcal{A} u+\mathcal{B} u\|, \quad \lambda>0
$$

for some $M \geq 1$, independent of $\lambda$, and for all $u \in D(\mathcal{A}) \cap D(\mathcal{B})$.

Suppose the hypotheses of Proposition 4.10 are fulfilled. Then the pair of operators $\left(\mathcal{L}_{\alpha}, \mathcal{A}\right)$ is $\lambda$-regular. Indeed, replacing the operator $A$ by $\lambda A, \lambda>0$, we obtain the following multiplier function

$$
m_{\lambda}(\rho):=\lambda A\left((i \rho)^{\alpha} I+\lambda A\right)^{-1}=A\left(\lambda^{-1}(i \rho)^{\alpha} I+A\right)^{-1} .
$$

Therefore $\mathcal{R}\left(\left\{m_{\lambda}(\rho) \mid \rho \in \mathbb{R} \backslash\{0\}\right\}\right)=\mathcal{R}(\{m(\rho) \mid \rho \in \mathbb{R} \backslash\{0\}\})=k_{0}$ and so, it does not depend on $\lambda$. Applying Theorem 4.8, the estimate

$$
\left\|\mathcal{L}_{\alpha} u\right\|_{L^{p}(I ; X)}+\|\lambda \mathcal{A} u\|_{L^{p}(I ; X)} \leq M\|f\|_{L^{p}(I ; X)}
$$

follows, where $M$ does not depend on $\lambda$.

Corollary 4.12 Conditions of Proposition 4.10 are sufficient for $\left(\mathcal{L}_{\alpha}, \mathcal{A}\right)$ to be a $\lambda$-regular pair in $L^{p}(I ; X)$ and in $L^{p}(\mathbb{R} ; X)$. 


\subsection{Strict $L^{p}$ solutions of fractional order equations}

Consider now the fractional evolution equations with nonzero initial conditions

$$
\begin{array}{ll}
\alpha \in(0,1): \quad & D_{t}^{\alpha} u(t)+A u(t)=f(t), \text { a.a. } t>0, \\
& \left(g_{1-\alpha} * u\right)(0)=x_{0} .
\end{array}
$$

and

$$
\begin{array}{ll}
\alpha \in(1,2): \quad & D_{t}^{\alpha} u(t)+A u(t)=f(t), \text { a.a. } t>0, \\
& \left(g_{2-\alpha} * u\right)(0)=x_{0},\left(g_{2-\alpha} * u\right)^{\prime}(0)=x_{1} .
\end{array}
$$

where $x_{0}, x_{1} \in X$ and $f \in L^{p}(I ; X)$.

Definition 4.13 A function $u: I \rightarrow X$ is said to be a strict $\boldsymbol{L}^{p}$ solution of (4.25), resp. (4.26), on $I$, in $X$, if $u \in L^{p}(I ; D(A)) \cap R^{\alpha, p}(I ; X)$ and (4.25), resp. (4.26), is satisfied.

Obviously, if $x_{0}=x_{1}=0$, then (4.25), resp. (4.26), has strict $L^{p}$ solution for any $f \in L^{p}$ iff it has maximal $L^{p}$ regularity.

In order to solve (4.25), we write $u=v+w$, where $v$ satisfies

$$
\begin{aligned}
& D_{t}^{\alpha} v(t)+A v(t)=f(t), \text { a.a. } t>0, \\
& \left(g_{1-\alpha} * v\right)(0)=0 .
\end{aligned}
$$

and $w$ satisfies

$$
\begin{aligned}
& D_{t}^{\alpha} w(t)+A w(t)=0, \text { a.a. } t>0, \\
& \left(g_{1-\alpha} * w\right)(0)=x_{0} .
\end{aligned}
$$

Similarly, in order to solve (4.26), we write $u=v+w+z$, where $v$ satisfies

$$
\begin{aligned}
& D_{t}^{\alpha} v(t)+A v(t)=f(t), \text { a.a. } t>0, \\
& \left(g_{2-\alpha} * v\right)(0)=0,\left(g_{2-\alpha} * v\right)^{\prime}(0)=0,
\end{aligned}
$$

$w$ satisfies

$$
\begin{aligned}
& D_{t}^{\alpha} w(t)+A w(t)=0, \text { a.a. } t>0, \\
& \left(g_{2-\alpha} * w\right)(0)=x_{0}, \quad\left(g_{2-\alpha} * w\right)^{\prime}(0)=0,
\end{aligned}
$$

and $z$ satisfies

$$
\begin{aligned}
& D_{t}^{\alpha} z(t)+A z(t)=0, \text { a.a. } t>0, \\
& \left(g_{2-\alpha} * z\right)(0)=0,\left(g_{2-\alpha} * z\right)^{\prime}(0)=x_{1} .
\end{aligned}
$$

We apply different methods to analyse the above problems. For the analysis of (4.27) and (4.29) we use the results on maximal $L^{p}$ regularity given in Proposition 4.10 and Corollary 4.6, while for the analysis of (4.28), (4.30) and (4.31) we use the solution operator $P_{\alpha}(t)$ associated with it, defined as follows. 
Let $A$ be a nonnegative operator with spectral angle $\omega_{A}$ satisfying (4.9). Define the operator-valued function

$$
P_{\alpha}(t) x:=\frac{1}{2 \pi i} \int_{\Gamma_{r, \theta}} e^{\lambda t}\left(\lambda^{\alpha} I+A\right)^{-1} x d \lambda
$$

where $\theta \in\left(\frac{\pi}{2}, \min \left\{\pi, \frac{\pi-\omega_{A}}{\alpha}\right\}\right)$ and

$$
\Gamma_{r, \theta}:=\left\{r e^{i \varphi} ;|\varphi| \leq \theta\right\} \cup\left\{\rho e^{i \theta} ; r \leq \rho<\infty\right\} \cup\left\{\rho e^{-i \theta} \mid r \leq \rho<\infty\right\} .
$$

The orientation of the contour is such that the argument does not decrease along it. This function corresponds to the solution operator $S_{\alpha}(t)$ in the case of equations with Caputo fractional derivative considered in Chapters 2 and 3. Next we summarize some properties of $P_{\alpha}(t)$.

Lemma 4.14 Assume that $\alpha \in(0,2)$ and $A$ is a nonnegative operator in a complex Banach space $X$ with spectral angle $\omega_{A}$ satisfying (4.9). Then the following assertions hold

(a) $P_{\alpha}(t) \in \mathcal{B}(X)$ for each $t>0$ and $\sup _{t>0} t^{1-\alpha}\left\|P_{\alpha}(t)\right\|_{\mathcal{B}(X)}<\infty$;

(b) For any $x \in X, t>0, P_{\alpha}(t) x \in D(A)$ and $\sup _{t>0} t\left\|A P_{\alpha}(t)\right\|_{\mathcal{B}(X)}<\infty$;

(c) $P_{\alpha}(),. A P_{\alpha}^{(k)}(.) \in C^{\infty}\left(\mathbb{R}_{+} ; \mathcal{B}(X)\right)$ and for any integer $k \geq 0$ and $l=0,1$,

$$
\sup _{t>0} t^{1+k+\alpha(l-1)}\left\|A^{l} P_{\alpha}^{(k)}(t)\right\|_{\mathcal{B}(X)}<\infty
$$

(d) For any fixed $\theta \in\left(0, \min \left\{\pi, \frac{\pi-\omega_{A}}{\alpha}\right\}-\frac{\pi}{2}\right), k \geq 0, l=0,1$ there exists an analytic extension of $A^{l} P_{\alpha}^{(k)}($.$) to \Sigma_{\theta}$.

These properties can be obtained in the same spirit as the properties of $S_{\alpha}(t)$ in Theorem 2.14 and Proposition 2.15. Note that $A$ is nonnegative with spectral angle $\omega_{A}$ satisfying (4.9) iff $-A \in \mathcal{A}^{\alpha}\left(\theta_{0}, 0\right)$ with $\theta_{0}=\left(\pi(1-\alpha / 2)-\omega_{A}\right) / \alpha$.

From the definition of $P_{\alpha}(t)$ it follows

$$
\left(\lambda^{\alpha} I+A\right)^{-1} x_{0}=\int_{0}^{\infty} e^{-\lambda t} P_{\alpha}(t) x_{0} d t
$$

The maximal $L^{p}$ regularity of (4.4) is equivalent to the boundedness in $L^{p}(I ; X)$ of the operator $M$, defined by

$$
M f(t):=\int_{0}^{t} A P_{\alpha}(t-s) f(s) d s,
$$

because from the variation of parameters formula for the solution $u$ of (4.4) we have $A u(t)=M f(t)$. The solutions of the equations with arbitrary initial conditions and zero forcing function can also be represented in terms of $P_{\alpha}(t)$. Using this representation, we formulate some results on existence and uniqueness of strict $L^{p}$ solutions. 


\section{The case $\alpha \in(0,1)$}

Our main results concerning the case $\alpha \in(0,1)$ are two theorems on strict $L^{p}$ solvability in $X$ and in real interpolation spaces $D_{A}(\delta, p)$, correspondingly. First we prove two lemmas about strict solvability of the equation with zero forcing function.

By (4.33) and the uniqueness of the Laplace transform it follows that $w(t):=P_{\alpha}(t) x_{0}$ satisfies (4.28). The following lemma gives sufficient conditions for $P_{\alpha}(t) x_{0}$ to be a strict solution of (4.28). In fact we prove a stronger result, which except for our application, is of independent interest, because it gives an equivalent norm in the interpolation spaces $D_{A}\left(\frac{p-1}{\alpha p}, p\right)$ in terms of the operator-valued function $A P_{\alpha}(t)$.

Lemma 4.15 Assume that $\alpha \in(0,1)$ and $A$ is a nonnegative operator in a complex Banach space $X$ with spectral angle $\omega_{A}$ satisfying (4.9). Then the following assertions hold

(a) Let $1<p<\frac{1}{1-\alpha}$. Then $A P_{\alpha}(t) x_{0} \in L^{p}\left(\mathbb{R}_{+}, X\right)$ iff $x_{0} \in D_{A}\left(\frac{p-1}{\alpha p}, p\right)$. In this case there are constants $C_{1}, C_{2}$, depending only on $\alpha, p, \omega_{A}$ and $K_{A}(\phi)$ for some $\phi \in$ $\left(\alpha \pi / 2, \pi-\omega_{A}\right)$, such that

$$
C_{1}\left[x_{0}\right]_{D_{A}\left(\frac{p-1}{\alpha p}, p\right)} \leq\left\|A P_{\alpha}(t) x_{0}\right\|_{L^{p}\left(\mathbb{R}_{+} ; X\right)} \leq C_{2}\left[x_{0}\right]_{D_{A}\left(\frac{p-1}{\alpha p}, p\right)}
$$

(b) Let $p \geq \frac{1}{1-\alpha}$. Then $A P_{\alpha}(t) x_{0} \in L^{p}\left(\mathbb{R}_{+}, X\right)$ iff $x_{0}=0$.

Proof: Let $1<p<\frac{1}{1-\alpha}$ and $x_{0} \in D_{A}\left(\frac{p-1}{\alpha p}, p\right)$. According to (4.32) and using analyticity to change the integration path we get, when we change the integration variable,

$$
A P_{\alpha}(t) x_{0}=\frac{1}{2 \pi i} \int_{\Gamma_{r, \theta}} e^{\mu} A\left(\frac{\mu^{\alpha}}{t^{\alpha}} I+A\right)^{-1} x_{0} \frac{d \mu}{t}, r>0 .
$$

By the dominated convergence theorem we can let $r \rightarrow 0$ and get

$$
A P_{\alpha}(t) x_{0}=\frac{e^{i \theta}}{\pi i} \int_{0}^{\infty} e^{\rho e^{i \theta}} A\left(\frac{\rho^{\alpha} e^{i \alpha \theta}}{t^{\alpha}} I+A\right)^{-1} x_{0} \frac{d \rho}{t} .
$$

To estimate the function under the integral sign we use the representation for $s>0$

$$
A\left(A+s e^{ \pm i \phi} I\right)^{-1}-A(A+s I)^{-1}=\left(e^{\mp i \phi}-1\right) s e^{ \pm i \phi}\left(A+s e^{ \pm i \phi} I\right)^{-1} A(A+s I)^{-1},
$$

which implies

$$
\left\|A(A+z I)^{-1} x_{0}\right\|_{X} \leq\left(1+2 \sin \frac{\phi}{2} K_{A}(\phi)\right)\left\|A(A+|z| I)^{-1} x_{0}\right\|_{X},|\arg z|=\phi .
$$

Therefore

$$
\left\|A P_{\alpha}(t) x_{0}\right\|_{X} \leq c_{1} \int_{0}^{\infty} e^{\rho \cos \theta}\left\|A\left(\frac{\rho^{\alpha}}{t^{\alpha}} I+A\right)^{-1} x_{0}\right\|_{X} \frac{d \rho}{t}
$$


where $c_{1}=\pi^{-1}\left(1+2 \sin \frac{\alpha \theta}{2} K_{A}(\alpha \theta)\right)$. Applying the generalized Minkowski inequality

$$
\left\{\int_{\mathbb{R}_{+}} d \tau\left|\int_{\mathbb{R}_{+}} f(\tau, t) d t\right|^{p}\right\}^{1 / p} \leq \int_{\mathbb{R}_{+}} d t\left\{\int_{\mathbb{R}_{+}}|f(\tau, t)|^{p} d \tau\right\}^{1 / p},
$$

where $f(\tau, t)$ is a measurable function, defined on $\mathbb{R}_{+} \times \mathbb{R}_{+}$such that the integrals on both sides are well defined, we obtain

$$
\begin{aligned}
\left\|A P_{\alpha}(t) x_{0}\right\|_{L^{p}\left(\mathbb{R}_{+}, X\right)} & \leq c_{1} \int_{0}^{\infty} e^{\rho \cos \theta}\left(\int_{0}^{\infty}\left(\left\|A\left(\frac{\rho^{\alpha}}{t^{\alpha}} I+A\right)^{-1} x_{0}\right\|_{X} \frac{1}{t}\right)^{p} d t\right)^{\frac{1}{p}} d \rho \\
& =c_{1} \int_{0}^{\infty} e^{\rho \cos \theta} \rho^{\frac{1}{p}-1} d \rho\left(\int_{0}^{\infty}\left\|\sigma^{1-\frac{1}{p}} A\left(\sigma^{\alpha} I+A\right)^{-1} x_{0}\right\|_{X}^{p} \frac{d \sigma}{\sigma}\right)^{\frac{1}{p}} \\
& =c_{2}\left[x_{0}\right]_{D_{A}\left(\frac{p-1}{\alpha p}, p\right)},
\end{aligned}
$$

with $c_{2}=c_{1} \Gamma(1 / p)(-\alpha \cos \theta)^{-1 / p}$ and we have used

$$
[x]_{D_{A}\left(\frac{\gamma}{\alpha}, p\right)}=\left(\alpha \int_{0}^{\infty}\left(t^{\gamma}\left\|A\left(t^{\alpha} I+A\right)^{-1} x\right\|_{X}\right)^{p} \frac{d t}{t}\right)^{\frac{1}{p}},
$$

easily obtained from (1.5) for $0<\gamma<\alpha, p \in(1, \infty)$.

Suppose now that $A P_{\alpha}(t) x_{0} \in L^{p}\left(\mathbb{R}_{+} ; X\right)$. Applying (4.33) and the generalized Minkowski inequality, we obtain when we change twice the integration variable

$$
\begin{aligned}
& \left(\int_{0}^{\infty}\left\|\lambda^{1-\frac{1}{p}} A\left(\lambda^{\alpha} I+A\right)^{-1} x_{0}\right\|_{X}^{p} \frac{d \lambda}{\lambda}\right)^{\frac{1}{p}} \\
= & \left(\int_{0}^{\infty} \lambda^{p-2}\left\|\int_{0}^{\infty} e^{-\lambda t} A P_{\alpha}(t) x_{0} d t\right\|_{X}^{p} d \lambda\right)^{\frac{1}{p}} \\
= & \left(\int_{0}^{\infty} \lambda^{-2}\left\|\int_{0}^{\infty} e^{-\tau} A P_{\alpha}\left(\frac{\tau}{\lambda}\right) x_{0} d \tau\right\|_{X}^{p} d \lambda\right)^{\frac{1}{p}} \\
\leq & \int_{0}^{\infty} e^{-\tau}\left(\int_{0}^{\infty} \lambda^{-2}\left\|A P_{\alpha}\left(\frac{\tau}{\lambda}\right) x_{0}\right\|_{X}^{p} d \lambda\right)^{\frac{1}{p}} d \tau \\
= & \int_{0}^{\infty} e^{-\tau} \tau^{-\frac{1}{p}} d \tau\left(\int_{0}^{\infty}\left\|A P_{\alpha}(\sigma) x_{0}\right\|_{X}^{p} d \sigma\right)^{\frac{1}{p}} \\
= & \Gamma\left(1-\frac{1}{p}\right)\left\|A P_{\alpha}(t) x_{0}\right\|_{L^{p}\left(\mathbb{R}_{+}, X\right)}<\infty .
\end{aligned}
$$

Therefore, if $1<p<\frac{1}{1-\alpha},(4.40)$ and (4.41) imply $\left[x_{0}\right]_{D_{A}\left(\frac{p-1}{\alpha p}, p\right)} \leq c\left\|A P_{\alpha}(t) x_{0}\right\|_{L^{p}\left(\mathbb{R}_{+}, X\right)}<$ $\infty$ and thus $x_{0} \in D_{A}\left(\frac{p-1}{\alpha p}, p\right)$. If $p \geq \frac{1}{1-\alpha}$ then (4.40) and (4.41) implies $x_{0}=0$, because $D_{A}(1, p)=\{0\}$.

In case $0 \in \rho(A)$, we can take $\|\cdot\|_{D_{A}(\delta, p)}=[\cdot]_{D_{A}(\delta, p)}$ as an equivalent norm in $D_{A}(\delta, p)$ (see [14]) and inequalities (4.34) imply

$$
C_{1}\left\|x_{0}\right\|_{D_{A}\left(\frac{p-1}{\alpha p}, p\right)} \leq\left\|A P_{\alpha}(t) x_{0}\right\|_{L^{p}\left(\mathbb{R}_{+} ; X\right)} \leq C_{2}\left\|x_{0}\right\|_{D_{A}\left(\frac{p-1}{\alpha p}, p\right)} .
$$


Moreover, Lemma 4.14 (b) implies

$\int_{0}^{\infty}\left\|A P_{\alpha}(t)\right\|_{X}^{p} d t=\int_{0}^{T}\left\|A P_{\alpha}(t)\right\|_{X}^{p} d t+\int_{T}^{\infty}\left\|A P_{\alpha}(t)\right\|_{X}^{p} d t \leq \int_{0}^{T}\left\|A P_{\alpha}(t)\right\|_{X}^{p} d t+\int_{T}^{\infty} \frac{C^{p}}{t^{p}} d t$

Since $p>1$ then $A P_{\alpha}(t) x_{0} \in L^{p}(I ; X)$ is equivalent to $A P_{\alpha}(t) x_{0} \in L^{p}\left(\mathbb{R}_{+} ; X\right)$. These results together with Proposition 4.10 imply the following theorem.

Theorem 4.16 Suppose that $\alpha \in(0,1), 1<p<\infty, X$ is a Banach space of class $\mathcal{H} \mathcal{T}$, $A$ is an $\mathcal{R}$-sectorial operator in $X$ with $0 \in \rho(A)$ and with $\mathcal{R}$-angle $\omega_{A}^{R}$, satisfying (4.20), and $f \in L^{p}(I ; X)$. Then the following statements hold:

(a) if $1<p<\frac{1}{1-\alpha}$, then there is a unique strict $L^{p}$ solution $u$ of $(4.25)$ iff $x_{0} \in D_{A}\left(\frac{p-1}{\alpha p}, p\right)$;

(b) if $p \geq \frac{1}{1-\alpha}$ then (4.25) has a unique strict $L^{p}$ solution iff $x_{0}=0$.

In both cases the following estimate is satisfied (for (b) we set $x_{0}=0$ ):

$$
\|u\|_{L^{p}(I ; X)}+\left\|D_{t}^{\alpha} u\right\|_{L^{p}(I ; X)}+\|A u\|_{L^{p}(I ; X)} \leq C\left(\left\|x_{0}\right\|_{D_{A}\left(\frac{p-1}{\alpha p}, p\right)}+\|f\|_{L^{p}(I ; X)}\right),
$$

where the constant $C$ depends on $X, \alpha, p, \omega_{A}$ and $K_{A}(\theta)$ for some $\theta \in\left(\alpha \pi / 2, \pi-\omega_{A}\right)$ and on $\mathcal{R}_{A}(\alpha \pi / 2)$, but does not depend on $T$ and on the individual operator $A$.

To obtain further regularity results we need more detailed estimates on $A P_{\alpha}(t) x_{0}$. Next we present conditions under which $A P_{\alpha}(t) x_{0}$ belongs to some interpolation spaces.

Lemma 4.17 Assume that $\alpha \in(0,1)$ and $A$ is a nonnegative operator in a complex Banach space $X$ with spectral angle $\omega_{A}$ satisfying (4.9). If $1<p<\frac{1}{1-\alpha}, 0<\delta<\frac{\alpha p-p+1}{\alpha p}$ and $x_{0} \in D_{A}\left(\frac{p-1}{\alpha p}+\delta, p\right)$ then $A P_{\alpha}(t) x_{0} \in L^{p}\left(\mathbb{R}_{+} ; D_{A}(\delta, p)\right)$. More precisely, there is a constant $C$ depending on $\alpha, \delta, p, \omega_{A}$ and $K_{A}(\phi)$ for some $\phi \in\left(\alpha \pi / 2, \pi-\omega_{A}\right)$, such that

$$
\left\|A P_{\alpha}(t) x_{0}\right\|_{L^{p}\left(\mathbb{R}_{+} ; D_{A}(\delta, p)\right)} \leq C\left\|x_{0}\right\|_{D_{A}\left(\frac{p-1}{\alpha p}+\delta, p\right)} .
$$

Proof: Set $\gamma=\alpha \delta$. According to (4.32) we get

$$
A P_{\alpha}(t) x_{0}=\frac{1}{2 \pi i} \int_{\Gamma_{r, \theta}} e^{\lambda t} A\left(\lambda^{\alpha} I+A\right)^{-1} x_{0} d \lambda, r>0 .
$$

Take $\mu^{\alpha}>r$. Since

$$
A\left(\mu^{\alpha} I+A\right)^{-1} A\left(\lambda^{\alpha} I+A\right)^{-1}=\frac{\mu^{\alpha}}{\mu^{\alpha}-\lambda^{\alpha}} A\left(\mu^{\alpha} I+A\right)^{-1}-\frac{\lambda^{\alpha}}{\mu^{\alpha}-\lambda^{\alpha}} A\left(\lambda^{\alpha} I+A\right)^{-1},
$$

it follows by (4.45)

$$
A\left(\mu^{\alpha} I+A\right)^{-1} A P_{\alpha}(t) x_{0}
$$




$$
=A\left(\mu^{\alpha} I+A\right)^{-1} \frac{1}{2 \pi i} \int_{\Gamma_{r, \theta}} \frac{\mu^{\alpha}}{\mu^{\alpha}-\lambda^{\alpha}} e^{\lambda t} x_{0} d \lambda-\frac{1}{2 \pi i} \int_{\Gamma_{r, \theta}} \frac{\lambda^{\alpha}}{\mu^{\alpha}-\lambda^{\alpha}} e^{\lambda t} A\left(\lambda^{\alpha} I+A\right)^{-1} x_{0} d \lambda .
$$

When we close the path $\Gamma_{r, \theta}$ at infinity by increasing argument, we see that the first integral is 0 by Cauchy's theorem and we get

$$
A\left(\mu^{\alpha} I+A\right)^{-1} A P_{\alpha}(t) x_{0}=-\frac{1}{2 \pi i} \int_{\Gamma_{r, \theta}} \frac{\lambda^{\alpha}}{\mu^{\alpha}-\lambda^{\alpha}} e^{\lambda t} A\left(\lambda^{\alpha} I+A\right)^{-1} x_{0} d \lambda
$$

In this integral we may let $r \downarrow 0$ without changing the value of the integral, because the function we integrate is analytic and the integral over a part of the circle with radius $r$ goes to 0 by the assumption that $\theta \in\left(\frac{\pi}{2}, \min \left\{\pi, \frac{\pi-\omega_{A}}{\alpha}\right\}\right)$, and the definition of $\Gamma_{r, \theta}$.

Thus we have by (4.46) and (4.37)

$$
\begin{aligned}
& \mu^{\gamma}\left\|A\left(\mu^{\alpha} I+A\right)^{-1} A P_{\alpha}(t) x_{0}\right\|_{X} \\
\leq & c_{1} \int_{0}^{\infty} \frac{\mu^{\gamma} s^{\alpha}}{\left|\mu^{\alpha}-s^{\alpha} e^{i \alpha \theta}\right|} e^{s t \cos \theta}\left\|A\left(s^{\alpha} I+A\right)^{-1} x_{0}\right\|_{X} d s \\
= & c_{1} \int_{0}^{\infty} \frac{\left(\frac{\mu}{s}\right)^{\gamma}}{\left|\left(\frac{\mu}{s}\right)^{\alpha}-e^{i \alpha \theta}\right|} s^{\gamma+1} e^{s t \cos \theta}\left\|A\left(s^{\alpha} I+A\right)^{-1} x_{0}\right\|_{X} \frac{d s}{s},
\end{aligned}
$$

where $c_{1}=\pi^{-1}\left(1+2 \sin \frac{\alpha \theta}{2} K_{A}(\alpha \theta)\right)$. Let for $\tau \in \mathbb{R}$

$$
\begin{gathered}
f(\tau):=e^{\tau(\gamma+1)} e^{e^{\tau} t \cos \theta}\left\|A\left(e^{\tau \alpha} I+A\right)^{-1} x_{0}\right\|_{X}, \quad g(\tau):=e^{\tau \gamma}\left\|A\left(e^{\tau \alpha} I+A\right)^{-1} A P_{\alpha}(t) x_{0}\right\|_{X}, \\
h(\tau):=e^{\tau \gamma} /\left|e^{\tau \alpha}-e^{i \alpha \theta}\right| .
\end{gathered}
$$

By changing variables $\left(s=e^{\sigma}\right)$ in the integral in (4.47) we conclude that

$$
g(\tau) \leq c_{1} \int_{-\infty}^{\infty} h(\tau-\sigma) f(\sigma) d \sigma
$$

Since $h \in L^{1}(\mathbb{R}), f \in L^{p}(\mathbb{R} ; X)$, we can apply the Young inequality to (4.48) to obtain $\|g\|_{L^{p}(\mathbb{R} ; X)} \leq c_{1}\|h\|_{L^{1}(\mathbb{R})}\|f\|_{L^{p}(\mathbb{R} ; X)}$. Because a change of variables shows that $\|g\|_{L^{p}(\mathbb{R})}=$ $\left[A P_{\alpha}(t) x_{0}\right]_{D_{A}(\delta, p)}$, we conclude after another change of variables that

$$
\left[A P_{\alpha}(t) x_{0}\right]_{D_{A}(\delta, p)} \leq c_{1} \int_{0}^{\infty} \frac{s^{\gamma-1}}{\left|s^{\alpha}+e^{i \alpha \theta}\right|} d s\left(\int_{0}^{\infty}\left(s^{\gamma+1} e^{s t \cos \theta}\left\|A\left(s^{\alpha} I+A\right)^{-1} x_{0}\right\|_{X}\right)^{p} \frac{d s}{s}\right)^{\frac{1}{p}} .
$$

Therefore, setting $c_{2}=c_{1} \int_{0}^{\infty} \frac{s^{\gamma-1}}{\left|s^{\alpha}+e^{i \alpha \theta}\right|} d s$, we obtain

$$
\begin{aligned}
& \left(\int_{0}^{\infty}\left[A P_{\alpha}(t) x_{0}\right]_{D_{A}(\delta, p)}^{p} d t\right)^{\frac{1}{p}} \\
\leq & c_{2}\left(\int_{0}^{\infty} \int_{0}^{\infty}\left(s^{\gamma+1} e^{s t \cos \theta}\left\|A\left(s^{\alpha} I+A\right)^{-1} x_{0}\right\|_{X}\right)^{p} \frac{d s}{s} d t\right)^{\frac{1}{p}} \\
= & c_{2}\left(\int_{0}^{\infty} \int_{0}^{\infty}\left(\left(\frac{\sigma}{t}\right)^{\gamma+1} e^{\sigma \cos \theta}\left\|A\left(\left(\frac{\sigma}{t}\right)^{\alpha} I+A\right)^{-1} x_{0}\right\|_{X}\right)^{p} \frac{d \sigma}{\sigma} d t\right)^{\frac{1}{p}}
\end{aligned}
$$




$$
\begin{aligned}
& =c_{2}\left(\int_{0}^{\infty}\left(\sigma^{\gamma+1-\frac{1}{p}} e^{\sigma \cos \theta}\right)^{p} d \sigma \int_{0}^{\infty}\left(t^{-\gamma-1}\left\|A\left(\left(\frac{\sigma}{t}\right)^{\alpha} I+A\right)^{-1} x_{0}\right\|_{X}\right)^{p} d t\right)^{\frac{1}{p}} \\
& =c_{2}\left(\int_{0}^{\infty} e^{\sigma p \cos \theta} d \sigma \int_{0}^{\infty}\left(\tau^{\gamma+1-\frac{1}{p}}\left\|A\left(\tau^{\alpha} I+A\right)^{-1} x_{0}\right\|_{X}\right)^{p} \frac{d \tau}{\tau}\right)^{\frac{1}{p}} \\
& =c_{3}\left[x_{0}\right]_{D_{A}\left(\frac{\gamma+1-\frac{1}{p}}{\alpha}, p\right)}=c_{3}\left[x_{0}\right]_{D_{A}\left(\frac{p-1}{\alpha p}+\delta, p\right)} \leq c_{3}\left\|x_{0}\right\|_{D_{A}\left(\frac{p-1}{\alpha p}+\delta, p\right)},
\end{aligned}
$$

where $c_{3}=c_{2}(-p \cos \theta)^{-\frac{1}{p}}$. By (4.49), (4.42) and the embedding

$$
D_{A}\left(\frac{p-1}{\alpha p}+\delta, p\right) \hookrightarrow D_{A}\left(\frac{p-1}{\alpha p}, p\right)
$$

(i.e. $\left.\left\|x_{0}\right\|_{D_{A}\left(\frac{p-1}{\alpha p}, p\right)} \leq c\left\|x_{0}\right\|_{D_{A}\left(\frac{p-1}{\alpha p}+\delta, p\right)}\right)$, we obtain (4.44).

This lemma shows that if $x_{0} \in D_{A}\left(\frac{p-1}{\alpha p}+\delta, p\right)$ then $P_{\alpha}(t) x_{0}$ is a strict $L^{p}$ solution of $(4.28)$ in $D_{A}(\delta, p)$. This result together with Corollary 4.6 (a) implies the following theorem:

Theorem 4.18 Suppose that $\alpha \in(0,1), 1<p<\infty, A$ is a positive operator in a Banach space $X$ with spectral angle $\omega_{A}$, satisfying (4.9). If $1<p<\frac{1}{1-\alpha}, 0<\delta<\frac{\alpha p-p+1}{\alpha p}$, $x_{0} \in D_{A}\left(\frac{p-1}{\alpha p}+\delta, p\right), f \in L^{p}\left(I ; D_{A}(\delta, p)\right)$, then there is a unique strict $L^{p}$ solution $u$ of (4.25) in $D_{A}(\delta, p)$ satisfying

$\|u\|_{L^{p}\left(I ; D_{A}(\delta, p)\right)}+\left\|D_{t}^{\alpha} u\right\|_{L^{p}\left(I ; D_{A}(\delta, p)\right)}+\|A u\|_{L^{p}\left(I ; D_{A}(\delta, p)\right)} \leq C\left(\left\|x_{0}\right\|_{D_{A}\left(\frac{p-1}{\alpha p}+\delta, p\right)}+\|f\|_{L^{p}\left(I ; D_{A}(\delta, p)\right)}\right)$.

This result holds also if $\frac{\alpha p-p+1}{\alpha p} \leq \delta<1$ and $x_{0}=0$. The constant $C$ depends on $X, \alpha, p, \delta, \omega_{A}$ and $K_{A}(\theta)$ for some $\theta \in\left(\alpha \pi / 2, \pi-\omega_{A}\right)$, but does not depend on $T$ and on the individual operator $A$.

Lemma 4.17 concerns "spatial regularity". We expect that the following result concerning "temporal regularity" also holds. If it is true, it could be combined with Corollary 4.6 (b) to obtain a result analogous to Theorem 4.18.

Conjecture: Under the conditions of Lemma 4.17 the following statements hold

(a) If $1 \leq p<\frac{1}{1-\alpha}, 0<\delta<\frac{\alpha p-p+1}{\alpha p}$ then $A P_{\alpha}(t) x_{0} \in W^{\alpha \delta, p}\left(\mathbb{R}_{+} ; X\right)$ iff $x_{0} \in D_{A}\left(\frac{p-1}{\alpha p}+\delta, p\right)$. In this case there are constants $C_{1}, C_{2}$ such that

$$
C_{1}\left[x_{0}\right]_{D_{A}\left(\frac{p-1}{\alpha p}+\delta, p\right)} \leq\left\|A P_{\alpha}(t) x_{0}\right\|_{W^{\alpha \delta, p}\left(\mathbb{R}_{+} ; X\right)} \leq C_{2}\left[x_{0}\right]_{D_{A}\left(\frac{p-1}{\alpha p}+\delta, p\right)}
$$

(b) If $1 \leq p<\frac{1}{1-\alpha}, \delta \geq \frac{\alpha p-p+1}{\alpha p}$ then $A P_{\alpha}(t) x_{0} \in W^{\alpha \delta, p}\left(\mathbb{R}_{+} ; X\right)$ iff $x_{0}=0$.

We give the proof of the "only if" part of (a) and (b). If $A P_{\alpha}(t) x \in W^{\alpha \delta, p}\left(\mathbb{R}_{+} ; X\right)$, we obtain as in (4.41)

$$
\begin{aligned}
{\left[x_{0}\right]_{D_{A}\left(\frac{p-1}{\alpha p}+\delta, p\right)} } & =\left(\int_{0}^{\infty}\left\|\lambda^{1-\frac{1}{p}+\alpha \delta} A\left(\lambda^{\alpha} I+A\right)^{-1} x_{0}\right\|_{X}^{p} \frac{d \lambda}{\lambda}\right)^{\frac{1}{p}} \\
& =\Gamma\left(1-\frac{1}{p}+\alpha \delta\right)\left(\int_{0}^{\infty} \sigma^{-p \alpha \delta}\left\|A P_{\alpha}(\sigma) x_{0}\right\|_{X}^{p} d \sigma\right)^{\frac{1}{p}} \\
& \leq c\left\|A P_{\alpha}(t) x_{0}\right\|_{W^{\alpha \delta, p}\left(\mathbb{R}_{+} ; X\right)}<\infty .
\end{aligned}
$$


Therefore, $x_{0} \in D_{A}\left(\frac{p-1}{\alpha p}+\delta, p\right)$ if $\frac{p-1}{\alpha p}+\delta<1$, otherwise $x_{0}=0$.

\section{The case $\alpha \in(1,2)$}

Applying the Laplace transform to the equations (4.30) and (4.31) we obtain formally that the Laplace transforms of their solutions $w$ and $z$ are $\lambda\left(\lambda^{\alpha} I+A\right)^{-1} x_{0}$ and $\left(\lambda^{\alpha} I+A\right)^{-1} x_{1}$, respectively. Therefore, besides $P_{\alpha}(t)$, defined as in (4.32), we consider also $Q_{\alpha}(t)$, defined by

$$
Q_{\alpha}(t) x:=\frac{1}{2 \pi i} \int_{\Gamma_{r, \theta}} e^{\lambda t} \lambda\left(\lambda^{\alpha} I+A\right)^{-1} x d \lambda
$$

with the same integration path as $P_{\alpha}(t)$. The properties of $Q_{\alpha}(t)$ can be derived from Lemma 4.14 and the fact that $Q_{\alpha}(t)=P_{\alpha}^{\prime}(t)$. It is not difficult to check that $w(t):=$ $Q_{\alpha}(t) x_{0}$ satisfies (4.30) and $z(t):=P_{\alpha}(t) x_{1}$ satisfies (4.31). More information about regularity of these solutions is contained in the following two lemmas, which can be proven in the same way as Lemmas 4.15 and 4.17, so we omit their proofs.

Lemma 4.19 Assume that $\alpha \in(1,2)$ and $A$ is a nonnegative operator in a complex Banach space $X$ with spectral angle $\omega_{A}$ satisfying (4.9). Then the following assertions hold

(a) Let $1<p<\frac{1}{2-\alpha}$. Then $A Q_{\alpha}(t) x_{0} \in L^{p}\left(\mathbb{R}_{+} ; X\right)$ iff $x_{0} \in D_{A}\left(\frac{2 p-1}{\alpha p}, p\right)$. In this case there are constants $C_{1}$ and $C_{2}$, such that

$$
C_{1}\left[x_{0}\right]_{D_{A}\left(\frac{2 p-1}{\alpha p}, p\right)} \leq\left\|A Q_{\alpha}(t) x_{0}\right\|_{L^{p}\left(\mathbb{R}_{+} ; X\right)} \leq C_{2}\left[x_{0}\right]_{D_{A}\left(\frac{2 p-1}{\alpha p}, p\right)}
$$

(b) Let $p \geq \frac{1}{2-\alpha}$. Then $A Q_{\alpha}(t) x_{0} \in L^{p}\left(\mathbb{R}_{+} ; X\right)$ iff $x_{0}=0$.

(c) For all $p>1, A P_{\alpha}(t) x_{1} \in L^{p}\left(\mathbb{R}_{+} ; X\right)$ iff $x_{1} \in D_{A}\left(\frac{p-1}{\alpha p}, p\right)$. In this case there are constants $C_{1}^{\prime}$ and $C_{2}^{\prime}$, such that

$$
C_{1}^{\prime}\left[x_{1}\right]_{D_{A}\left(\frac{p-1}{\alpha p}, p\right)} \leq\left\|A P_{\alpha}(t) x_{1}\right\|_{L^{p}\left(\mathbb{R}_{+} ; X\right)} \leq C_{2}^{\prime}\left[x_{1}\right]_{D_{A}\left(\frac{p-1}{\alpha p}, p\right)}
$$

The constants depend on $\alpha, p, \omega_{A}$ and $K_{A}(\theta)$ for some $\theta \in\left(\alpha \pi / 2, \pi-\omega_{A}\right)$.

Lemma 4.20 Assume that $\alpha \in(1,2)$ and $A$ is a nonnegative operator in a complex Banach space $X$ with spectral angle $\omega_{A}$ satisfying (4.9). Then the following assertions hold

(a) If $1<p<\frac{1}{2-\alpha}, 0<\delta<\frac{\alpha p-2 p+1}{\alpha p}$ and $x_{0} \in D_{A}\left(\frac{2 p-1}{\alpha p}+\delta, p\right)$ then $Q_{\alpha}(t) x_{0}$ is a strict solution of (4.30) in $L^{p}\left(\mathbb{R}_{+} ; D_{A}(\theta, p)\right)$, satisfying

$$
\left\|A Q_{\alpha}(t) x_{0}\right\|_{L^{p}\left(\mathbb{R}_{+} ; D_{A}(\delta, p)\right)} \leq C\left\|x_{0}\right\|_{D_{A}\left(\frac{2 p-1}{\alpha p}+\delta, p\right)} .
$$


(b) If $p>1,0<\delta<\frac{\alpha p-p+1}{\alpha p}$ and $x_{1} \in D_{A}\left(\frac{p-1}{\alpha p}+\delta\right.$, $\left.p\right)$ then $P_{\alpha}(t) x_{1}$ is a strict solution of (4.31) in $L^{p}\left(\mathbb{R}_{+} ; D_{A}(\theta, p)\right)$ and

$$
\left\|A P_{\alpha}(t) x_{1}\right\|_{L^{p}\left(\mathbb{R}_{+} ; D_{A}(\delta, p)\right)} \leq C^{\prime}\left\|x_{1}\right\|_{D_{A}\left(\frac{p-1}{\alpha p}+\delta, p\right)} .
$$

The constants depend on $\alpha, \delta, p, \omega_{A}$ and $K_{A}(\theta)$ for some $\theta \in\left(\alpha \pi / 2, \pi-\omega_{A}\right)$.

Lemma 4.19 together with Proposition 4.10 implies the following theorem:

Theorem 4.21 Let $\alpha \in(1,2), 1<p<\infty, X$ be a Banach space of class $\mathcal{H} \mathcal{T}, A$ be an $\mathcal{R}$-sectorial operator in $X$ with $0 \in \rho(A)$ and with $\mathcal{R}$-angle $\omega_{A}^{R}$, satisfying (4.20), and $f \in L^{p}(I ; X)$. If $1<p<\frac{1}{2-\alpha}, x_{0} \in D_{A}\left(\frac{2 p-1}{\alpha p}, p\right)$ and $x_{1} \in D_{A}\left(\frac{p-1}{\alpha p}, p\right)$, then there is a unique strict $L^{p}$ solution $u$ of (4.26) satisfying

$$
\|u\|_{L^{p}(I ; X)}+\left\|D_{t}^{\alpha} u\right\|_{L^{p}(I ; X)}+\|A u\|_{L^{p}(I ; X)} \leq C\left(\left\|x_{0}\right\|_{D_{A}\left(\frac{2 p-1}{\alpha p}, p\right)}+\left\|x_{1}\right\|_{D_{A}\left(\frac{p-1}{\alpha p}, p\right)}+\|f\|_{L^{p}(I ; X)}\right)
$$

This result holds also if $p \geq \frac{1}{2-\alpha}, x_{0}=0$ and $x_{1} \in D_{A}\left(\frac{p-1}{\alpha p}, p\right)$. The constant $C$ has the same properties as in Theorem 4.16 .

Lemma 4.20 together with Corollary 4.6 imply the following theorem.

Theorem 4.22 Suppose that $\alpha \in(1,2), 1<p<\infty, A$ is a positive operator in a Banach space $X$ with spectral angle $\omega_{A}$, satisfying (4.9), and $f \in L^{p}\left(I ; D_{A}(\delta, p)\right)$. If $1<p<\frac{1}{2-\alpha}, 0<\delta<\frac{\alpha p-2 p+1}{\alpha p}, x_{0} \in D_{A}\left(\frac{2 p-1}{\alpha p}+\delta, p\right), x_{1} \in D_{A}\left(\frac{p-1}{\alpha p}+\delta, p\right)$, then there is a unique strict solution $u$ of $(4.26)$ in $L^{p}\left(I ; D_{A}(\delta, p)\right)$ satisfying

$$
\begin{gathered}
\|u\|_{L^{p}\left(I ; D_{A}(\delta, p)\right)}+\left\|D_{t}^{\alpha} u\right\|_{L^{p}\left(I ; D_{A}(\delta, p)\right)}+\|A u\|_{L^{p}\left(I ; D_{A}(\delta, p)\right)} \\
\leq C\left(\left\|x_{0}\right\|_{D_{A}\left(\frac{2 p-1}{\alpha p}+\delta, p\right)}+\left\|x_{1}\right\|_{D_{A}\left(\frac{p-1}{\alpha p}+\delta, p\right)}+\|f\|_{L^{p}\left(I ; D_{A}(\delta, p)\right)}\right) .
\end{gathered}
$$

This result holds also if $p \geq \frac{1}{2-\alpha}, 0<\delta<\frac{\alpha p-p+1}{\alpha p}, x_{0}=0, x_{1} \in D_{A}\left(\frac{p-1}{\alpha p}+\delta, p\right)$. The constant $C$ has the same properties as in Theorem 4.18.

In this way we obtained a complete picture of the strict $L^{p}$ solvability of fractional autonomous equations. Note that maximal regularity results in the setting of Hölder continuous functions instead of $L^{p}$ functions are obtained in [15] for $\alpha \in(0,1)$ and [16] for $\alpha \in(1,2)$. 


\section{Chapter 5}

\section{Maximal regularity in Hilbert spaces via accretivity}

If $\alpha \in(0,1)$ then $\mathcal{L}_{\alpha}$ are $m$-accretive operators. So, taking $A$ also accretive, we can apply the theory of sums of accretive operators (see e.g. [30]) to prove maximal regularity even for $A$ nonlinear. This is a simple real method, completely different from the theorems of Da Prato-Grisvard and Dore-Venni type. Since the operators $\mathcal{L}_{\alpha}$ with $\alpha \in(1,2)$ are no more accretive, we can not apply directly the method of sums of accretive operators in this case. On the other hand, it is well known that the reduction of a second order Cauchy problem to a first order system is an important tool in treating second order problems. An attempt to apply this idea to our case, rewriting the problem of order $\alpha \in(1,2)$ to a problem of order $\alpha / 2$ with an accretive operator, shows, however, that this is possible only when the original operator is self-adjoint.

Linear nonautonomous problems are also studied via accretivity. The obtained results are applied to the fractional Löwner-Kufarev equation.

\subsection{Two classical results}

Let $\alpha \in(0,2)$. Assume $H$ is a Hilbert space with inner product $(.,$.$) and A$ is a linear closed densely defined operator on $H$. Let $I=(0, T), T>0$, and consider again the problem

$$
D_{t}^{\alpha} u(t)+A u(t)=f(t), \text { a.a. } t \in I,
$$

with an initial condition $\left(g_{1-\alpha} * u\right)(0)=0$ when $\alpha \in(0,1)$ and two initial conditions $\left(g_{2-\alpha} * u\right)(0)=0$ and $\left(g_{2-\alpha} * u\right)^{\prime}(0)=0$ when $\alpha \in(1,2)$. Our goal is to study its maximal regularity in the Hilbert space $L^{2}(I ; H)$.

We can use the following two theorems on the sum of commuting operators in Hilbert space. The first is due to Grisvard [44], the second to Dore and Venni [28].

Theorem 5.1 Let $\mathcal{A}$ and $\mathcal{B}$ be two linear, resolvent commuting, nonnegative operators in a Hilbert space $\mathcal{H}$ with spectral angles $\omega_{\mathcal{A}}$ and $\omega_{\mathcal{B}}$, respectively, such that $\omega_{\mathcal{A}}+\omega_{\mathcal{B}}<\pi$ 
and let $0 \in \rho(\mathcal{A}) \cup \rho(\mathcal{B})$. Assume

$$
D_{\mathcal{A}}(\theta, 2)=D_{\mathcal{A}^{*}}(\theta, 2)
$$

for some $0<\theta<1$. Then $0 \in \rho(\mathcal{A}+\mathcal{B})$ and $\omega_{\mathcal{A}+\mathcal{B}} \leq \max \left(\omega_{\mathcal{A}}, \omega_{\mathcal{B}}\right)$.

Theorem 5.2 Let $\mathcal{A}$ and $\mathcal{B}$ be two linear, resolvent commuting, nonnegative operators in a Hilbert space $\mathcal{H}$ with spectral angles $\omega_{\mathcal{A}}$ and $\omega_{\mathcal{B}}$, respectively, such that $\omega_{\mathcal{A}}+\omega_{\mathcal{B}}<\pi$ and let $0 \in \rho(\mathcal{A}) \cup \rho(\mathcal{B})$. Assume

$$
\sup _{0 \leq|s| \leq 1}\left\|\mathcal{A}^{i s}\right\|<\infty
$$

Then $0 \in \rho(\mathcal{A}+\mathcal{B})$ and $\omega_{\mathcal{A}+\mathcal{B}} \leq \max \left(\omega_{\mathcal{A}}, \omega_{\mathcal{B}}\right)$.

Let us note that if condition (5.2) is satisfied, then (5.3) holds [75]. In [9] an example of an operator $\mathcal{A}$, satisfying (5.3), but not (5.2), is given. It has been shown in [49] that if $\mathcal{A}$ is $m$-accretive in a Hilbert space, then condition (5.3) holds.

Since $\mathcal{L}_{\alpha}$ are operators with bounded imaginary powers in $L^{2}(I ; H)$ and $0 \in \rho\left(\mathcal{L}_{\alpha}\right)$, we obtain the following result:

Corollary 5.3 Let $\alpha \in(0,2), T>0$, and $H$ be a Hilbert space. If $A$ is nonnegative with spectral angle $\omega_{A}<\pi(1-\alpha / 2)$ then (5.1) has maximal regularity in $L^{p}(I ; H)$.

This can be obtained also from Proposition 4.10, because in Hilbert space the $\mathcal{R}$-boundedness coincides with the uniform norm boundedness. So, the problem for the maximal regularity of $(5.1)$ in $L^{2}(I ; H)$ is completely solved. Our goal in this chapter is to apply a simpler real method to study its maximal regularity: the method of sums of accretive operators.

\subsection{The case of nonlinear $m$-accretive operators with $\alpha \in(0,1)$}

Since the method of sums of accretive operators works also for nonlinear operators, we prefer to formulate our results in this more general form. First we extend some definitions to the nonlinear case. Let $A$ be a nonlinear multivalued operator of $D(A) \subset H$ into $H$. Just as in the linear case the operator $A$ is called $\boldsymbol{m}$-accretive, if for all $\lambda>0$ the resolvent $(\lambda I+A)^{-1}$ is everywhere defined and $\left\|(\lambda I+A)^{-1}\right\| \leq \lambda^{-1}$. By expressing the last condition in terms of the inner product of $H$ it can be shown that the operator $A$ is $m$-accretive iff $A$ is monotone, that is

$$
\left(u_{1}-u_{2}, v_{1}-v_{2}\right) \geq 0, \text { for all } v_{i} \in A u_{i}, i=1,2,
$$

and the operator $\lambda I+A$ is surjective for each positive number $\lambda$ ( equivalently, for some $\lambda>0)$. 
We suppose that

$A$ is $m$-accretive and $0 \in A 0$

and

$$
f \in L^{2}(I ; H) .
$$

Consider the abstract fractional differential equation of order $\alpha \in(0,1)$

$$
\begin{aligned}
& D_{t}^{\alpha} u(t)+A u(t) \ni f(t), \quad \text { a.a. } t \in I, \\
& \left(g_{1-\alpha} * u\right)(0)=x_{0} .
\end{aligned}
$$

Define maximal $L^{2}$ regularity of (5.7) in the same way as for linear $A$ (Definition 4.3).

In this chapter we use a basic lemma [8], Lemme 2.4, which we prove now for completeness.

Lemma 5.4 Let $\mathcal{A}$ be m-accretive and let $\mathcal{B}$ be an accretive Lipschitz continuous everywhere defined operator in a Hilbert space $\mathcal{H}$. Then $\mathcal{A}+\mathcal{B}$ with $D(\mathcal{A}+\mathcal{B})=D(\mathcal{A})$ is m-accretive.

Proof: It is immediate that $\mathcal{A}+\mathcal{B}$ is accretive. Thus, in order to prove the m-accretivity of the operator $\mathcal{A}+\mathcal{B}$, we need to show that the equation

$$
\lambda u+\mathcal{A} u+\mathcal{B} u=f
$$

has a solution $u \in D(\mathcal{A}) \cap D(\mathcal{B})$ for all $\lambda>0$ and $f \in \mathcal{H}$. Just as in the linear case it suffices to prove this only for some $\lambda_{0}>0$. Equation (5.8) is equivalent to

$$
u=(\lambda I+\mathcal{A})^{-1}(f-\mathcal{B} u) .
$$

Now take $\lambda_{0}>0$ sufficiently large, such that $\left\|B u_{1}-B u_{2}\right\| \leq \lambda_{0} / 2\left\|u_{1}-u_{2}\right\|$. Since $\left\|\left(\lambda_{0} I+\mathcal{A}\right)^{-1}\right\| \leq \lambda_{0}^{-1}$ it follows that the right-hand side of equation (5.9) defines a strict contraction in $\mathcal{H}$ and therefore it admits a unique fixed point. Therefore (5.8) has a unique solution.

In [18] maximal regularity of a generalization of $(5.7)$ in $L^{2}(I ; H)$ is studied using the theory of sums of accretive operators in a real Hilbert space. We repeat here the argument used in a slightly improved and shortened form. We consider only the case of our specific kernel $g_{1-\alpha}$.

Theorem 5.5 Let $\alpha \in(0,1), H$ be a real Hilbert space and $A$ be a nonlinear multivalued operator in $H$. Assume the conditions (5.5) and (5.6) hold. Then there exists a unique function $u \in L^{2}(I ; D(A)) \cap H^{\alpha, 2}(I ; H)$, satisfying (5.7).

Proof: Define operators of fractional differentiation on $\mathbb{R}$ via Fourier transform. Let $\widetilde{u}$ denotes the Fourier transform of $u$. We define $L_{\alpha}$, the operator of fractional differentiation on $\mathbb{R}$, by

$$
\widetilde{L_{\alpha} u}(\omega):=(i \omega)^{\alpha} \widetilde{u}(\omega), \omega \in \mathbb{R} \backslash\{0\},
$$




$$
D\left(L_{\alpha}\right):=\left\{\left.u \in L^{2}(\mathbb{R} ; \mathbb{R})\left|\int_{\mathbb{R}}\right| \omega\right|^{2 \alpha}|\widetilde{u}(\omega)|^{2} d \omega<\infty\right\}=H^{\alpha, 2}(\mathbb{R} ; \mathbb{R}) .
$$

Denote by $M(\mathbb{R})$ (see [21]) the set of linear $m$-accretive operators $L$ in $L^{2}(\mathbb{R} ; \mathbb{R})$ such that the resolvent $(I+\lambda L)^{-1}$ is order preserving and contractive in $L^{p}(\mathbb{R} ; \mathbb{R})$ for all $p \in[1, \infty]$ and all $\lambda>0$.

Since $g_{1-\alpha}$ is nonnegative and nonincreasing on $\mathbb{R}_{+}$, it follows that $L_{\alpha} \in M(\mathbb{R})([22])$. Let $L_{\alpha}^{*}$ be the adjoint operator of $L_{\alpha}$. Via Fourier transform we obtain $D\left(L_{\alpha}\right)=D\left(L_{\alpha}^{*}\right)$ and $\left\|L_{\alpha} u\right\|_{L^{2}(\mathbb{R} ; \mathbb{R})}=\left\|L_{\alpha}^{*} u\right\|_{L^{2}(\mathbb{R} ; \mathbb{R})}$, hence $L_{\alpha}$ is a normal operator. Define $L_{\alpha, 0}=\frac{1}{2}\left(L_{\alpha}+L_{\alpha}^{*}\right)$ with $D\left(L_{\alpha, 0}\right)=D\left(L_{\alpha}\right)$. This operator is clearly symmetric. Since $L_{\alpha}$ is $m$-accretive, $\omega_{L_{\alpha}}<\pi / 2$. Similarly $L_{\alpha}^{*}$ is $m$-accretive and $\omega_{L_{\alpha}^{*}}=\omega_{L_{\alpha}}$. Therefore $\omega_{L_{\alpha}}+\omega_{L_{\alpha}^{*}}<\pi$. Since $L_{\alpha}$ is normal it is evident that

$$
D_{L_{\alpha}}(\theta, 2)=D_{L_{\alpha}^{*}}(\theta, 2), \quad \theta \in(0,1)
$$

and it follows from Theorem 5.1 that $L_{\alpha, 0}$ is $m$-accretive. The fact that the resolvent of $L_{\alpha, 0}$ is order preserving and contractive in $L^{p}(\mathbb{R} ; \mathbb{R})$ for all $p \in[1, \infty]$ and all $\lambda>0$ can be seen using the Trotter product formula [71]. And finally, since $L_{\alpha}$ is normal, we have in $L^{2}(\mathbb{R} ; \mathbb{R})$

$$
2\left(L_{\alpha} u,\left(L_{\alpha}+L_{\alpha}^{*}\right) u\right)=\left(L_{\alpha} u, L_{\alpha} u\right)+2\left(L_{\alpha} u, L_{\alpha}^{*} u\right)+\left(L_{\alpha}^{*} u, L_{\alpha}^{*} u\right) .
$$

In this way we have proved the following

Lemma 5.6 If $L_{\alpha}$ is defined as above, then

(a) $L_{\alpha}$ is a normal operator, $L_{\alpha} \in M(\mathbb{R})$;

(b) $L_{\alpha, 0}$ is a symmetric operator, $L_{\alpha, 0} \in M(\mathbb{R})$;

(c) $\left(L_{\alpha}, L_{\alpha, 0}\right)_{L^{2}(\mathbb{R} ; \mathbb{R})}=\left\|L_{\alpha, 0}\right\|_{L^{2}(\mathbb{R} ; \mathbb{R})}^{2}$.

Let $\lambda>0, A_{\lambda}$ denotes the Yosida approximation of $A, A_{\lambda}:=\frac{1}{\lambda}\left(I-(I+\lambda A)^{-1}\right) \subseteq$ $A(I+\lambda A)^{-1}$. Consider the approximating equations to $(5.7)$ in $L^{2}(I ; H)$

$$
\lambda u_{\lambda}(t)+\frac{d}{d t}\left(g_{1-\alpha} * u_{\lambda}\right)(t)+A_{\lambda} u_{\lambda}(t)=f(t), t \in I .
$$

Let

$$
f_{0}= \begin{cases}f, & t \in I \\ 0, & t \notin I\end{cases}
$$

and $\mathcal{L}_{\alpha}, \mathcal{L}_{\alpha, 0}, \mathcal{A}$, and $\mathcal{A}_{\lambda}$ denote the $m$-accretive extensions of $L_{\alpha}, L_{\alpha, 0}, A$, and $A_{\lambda}$, respectively, to $L^{2}(\mathbb{R} ; H)$. Then we have

$$
D\left(\mathcal{L}_{\alpha}\right)=D\left(\mathcal{L}_{\alpha, 0}\right)=\left\{\left.u \in L^{2}(\mathbb{R} ; H)\left|\int_{\mathbb{R}}\right| \omega\right|^{2 \alpha}\|\tilde{u}(\omega)\|_{H}^{2} d \omega<\infty\right\}=H^{\alpha, 2}(\mathbb{R} ; H) .
$$


Now rewrite $(5.11)$ in $L^{2}(\mathbb{R} ; H)$ as

$$
\lambda u_{\lambda}+\mathcal{L}_{\alpha} u_{\lambda}+\mathcal{A}_{\lambda} u_{\lambda}=f_{0} .
$$

Since $\mathcal{L}_{\alpha}$ is $m$-accretive, $\mathcal{A}_{\lambda}$-accretive, Lipschitz continuous, everywhere defined, by Lemma 5.4 this equation has, for each $\lambda>0$, a unique solution $u_{\lambda} \in D\left(\mathcal{L}_{\alpha}\right)$. It follows from its uniqueness that $u_{\lambda} \equiv 0, t<0$.

Take the inner product of $(5.13)$ with $\mathcal{L}_{\alpha, 0} u_{\lambda}$ to obtain in $L^{2}(\mathbb{R} ; H)$ :

$$
\lambda\left(u_{\lambda}, \mathcal{L}_{\alpha, 0} u_{\lambda}\right)+\left(\mathcal{L}_{\alpha} u_{\lambda}, \mathcal{L}_{\alpha, 0} u_{\lambda}\right)+\left(\mathcal{A}_{\lambda} u_{\lambda}, \mathcal{L}_{\alpha, 0} u_{\lambda}\right)=\left(f_{0}, \mathcal{L}_{\alpha, 0} u_{\lambda}\right) .
$$

Lemma 5.6 (c) implies

$$
\left(\mathcal{L}_{\alpha} u_{\lambda}, \mathcal{L}_{\alpha, 0} u_{\lambda}\right)_{L^{2}(\mathbb{R} ; H)}=\left\|\mathcal{L}_{\alpha, 0} u_{\lambda}\right\|_{L^{2}(\mathbb{R} ; H)} .
$$

By (5.6), (5.12), (5.15) and by the accretivity of $\mathcal{L}_{\alpha, 0}$ it follows

$$
\left\|\mathcal{L}_{\alpha, 0} u_{\lambda}\right\|_{L^{2}(\mathbb{R} ; H)}^{2}+\left(\mathcal{A}_{\lambda} u_{\lambda}, \mathcal{L}_{\alpha, 0} u_{\lambda}\right)_{L^{2}(\mathbb{R} ; H)} \leq c\left\|\mathcal{L}_{\alpha, 0} u_{\lambda}\right\|_{L^{2}(\mathbb{R} ; H)},
$$

where $c=\|f\|_{L^{2}(I ; H)}<\infty$. Next, from Lemma $5.6(\mathrm{~b})$ and (5.5) it follows ([30], Theorem 3.3.1.) that

$$
\left(\mathcal{A}_{\lambda} u_{\lambda}, \mathcal{L}_{\alpha, 0} u_{\lambda}\right)_{L^{2}(\mathbb{R} ; H)} \geq 0, \lambda>0,
$$

and this inequality together with (5.16) implies

$$
\sup _{\lambda>0}\left\|\mathcal{L}_{\alpha, 0} u_{\lambda}\right\|_{L^{2}(\mathbb{R} ; H)} \leq c<\infty .
$$

But, using the properties of the Fourier transform, we get

$$
\left\|\mathcal{L}_{\alpha, 0} u_{\lambda}\right\|_{L^{2}(\mathbb{R} ; H)}=\cos \alpha \frac{\pi}{2}\left\|\mathcal{L}_{\alpha} u_{\lambda}\right\|_{L^{2}(\mathbb{R} ; H)},
$$

therefore

$$
\sup _{\lambda>0}\left\|\mathcal{L}_{\alpha} u_{\lambda}\right\|_{L^{2}(\mathbb{R} ; H)} \leq c\left(\cos \alpha \frac{\pi}{2}\right)^{-1}<\infty
$$

Next we prove that

$$
\sup _{\lambda>0} \int_{0}^{T}\left\|u_{\lambda}(t)\right\|_{H}^{2} d t \leq c \Gamma(1-\alpha) T^{\alpha}<\infty .
$$

If $v \in R^{\alpha, 2}(I ; H)$ we obtain

$$
\int_{0}^{T}\left(\frac{d}{d t}\left(g_{1-\alpha} * v\right), v\right)_{H} d t=g_{1-\alpha}(T) \int_{0}^{T}\|v\|_{H}^{2} d t+\int_{0}^{T}\left(\frac{d}{d t}\left(\left(g_{1-\alpha}-g_{1-\alpha}(T)\right) * v\right), v\right)_{H} d t
$$

and because $g_{1-\alpha}(t)-g_{1-\alpha}(T)$ is positive, nonincreasing for $t \in I$, the second integral on the right hand side is nonnegative and so

$$
\int_{0}^{T}\left(\frac{d}{d t}\left(g_{1-\alpha} * v\right), v\right)_{H} d t \geq g_{1-\alpha}(T) \int_{0}^{T}\|v\|_{H}^{2} d t
$$


Now multiply (5.11) by $u_{\lambda}$, integrate over $I$ and use the accretivity of $A_{\lambda},(5.6)$ and (5.21) to obtain (5.20). From (5.6), (5.20) and (5.19) there follows

$$
\sup _{\lambda>0}\left\|A_{\lambda} u_{\lambda}\right\|_{L^{2}(I ; H)}<\infty .
$$

Next we show that $u_{\lambda}$ converges as $\lambda \downarrow 0$. By (5.11)

$$
\lambda u_{\lambda}-\mu u_{\mu}+\frac{d}{d t}\left(g_{1-\alpha} *\left(u_{\lambda}-u_{\mu}\right)\right)+A_{\lambda} u_{\lambda}-A_{\mu} u_{\mu}=0 .
$$

Take the scalar product of (5.23) and $u_{\lambda}-u_{\mu}$ in $L^{2}(I ; H)$ and let $\lambda, \mu \downarrow 0$. By (5.20),

$$
\lim \sup _{\lambda, \mu \downarrow 0}\left(\lambda u_{\lambda}-\mu u_{\mu}, u_{\lambda}-u_{\mu}\right)_{L^{2}(I ; H)}=0 .
$$

Furthermore, by (5.22), (5.24) and the monotonicity of $A$, denoting $R_{\lambda}:=(I+\lambda A)^{-1}$, we have

$$
\begin{aligned}
& \lim \sup _{\lambda, \mu \downarrow}\left(A_{\lambda} u_{\lambda}-A_{\mu} u_{\mu}, u_{\lambda}-u_{\mu}\right)_{L^{2}(I ; H)} \\
= & \lim \sup _{\lambda, \mu \downarrow}\left(A_{\lambda} u_{\lambda}-A_{\mu} u_{\mu}, \lambda A_{\lambda} u_{\lambda}-\mu A_{\mu} u_{\mu}+R_{\lambda} u_{\lambda}-R_{\mu} u_{\mu}\right)_{L^{2}(I ; H)} \\
= & \lim \sup _{\lambda, \mu \downarrow 0}\left(\left(A_{\lambda} u_{\lambda}-A_{\mu} u_{\mu}, \lambda A_{\lambda} u_{\lambda}-\mu A_{\mu} u_{\mu}\right)_{L^{2}(I ; H)}\right. \\
+ & \left.\left(A R_{\lambda} u_{\lambda}-A R_{\mu} u_{\mu}, R_{\lambda} u_{\lambda}-R_{\mu} u_{\mu}\right)_{L^{2}(I ; H)}\right) \geq 0 .
\end{aligned}
$$

Combining the last inequality with (5.23) and (5.24), we obtain

$$
\lim \sup _{\lambda, \mu \downarrow 0}\left(\frac{d}{d t}\left(g_{1-\alpha} *\left(u_{\lambda}-u_{\mu}\right)\right), u_{\lambda}-u_{\mu}\right)_{L^{2}(I ; H)} \leq 0 .
$$

So, by (5.21) and (5.25) we have

$$
\lim \sup _{\lambda, \mu \downarrow 0}\left\|u_{\lambda}-u_{\mu}\right\|_{L^{2}(I ; H)}=0 .
$$

By (5.26) there exists $u$ such that $u_{\lambda} \rightarrow u$ in $L^{2}(I ; H)$. It follows from (5.19) and (5.22) the existence of $v$ and $w$, such that $\mathcal{L}_{\alpha} u_{\lambda} \rightarrow v, A_{\lambda} u_{\lambda} \rightarrow w$, where $\rightarrow$ denotes the weak convergence in $L^{2}(I ; H)$. Since $\mathcal{L}_{\alpha}$ is linear and closed in $L^{2}(I ; H)$, it is also weakly closed and therefore $u \in D\left(\mathcal{L}_{\alpha}\right)$ and $\mathcal{L}_{\alpha} u=v$. It remains to use [8], Prop. 2.5, to get $w=A u$. This completes the existence part of the theorem.

To obtain the uniqueness, assume the contrary and use (5.21) together with monotonicity. $\square$ In the next section we use the specialization of this result to linear operators $A$.

\subsection{Reduction of problems with $\alpha \in(1,2)$}

Let $H$ be a real Hilbert space with inner product $(.,$.$) and A: D(A) \subset H \rightarrow H$ be a linear $m$-accretive operator, $0 \in \rho(A)$. Let $\alpha \in(1,2)$ and $f \in L^{2}(I ; H)$. Consider the problem

$$
\begin{aligned}
& D_{t}^{\alpha} u(t)+A u(t)=f(t), \quad \text { a.a. } t \in I, \\
& \left(g_{2-\alpha} * u\right)(0)=0,\left(g_{2-\alpha} * u\right)^{\prime}(0)=0 .
\end{aligned}
$$


Since in this case the operator of fractional differentiation is no longer $m$-accretive, we cannot apply directly the method of the previous section. A way to make the application of this method possible also for $\alpha \in(1,2)$ is to rewrite $(5.27)$ as a system of equations of order $\alpha / 2$, where the corresponding matrix is an $m$-accretive operator. Recall that this is a natural way to study equations of order $\alpha=2$ and many results for secondorder Cauchy problems can be obtained by rewriting them as first-order problems. In the present section we try to apply this idea to our case.

The properties of $A$ imply that $A^{1 / 2}$ is well defined and we can define the Hilbert space $\mathcal{H}=D\left(A^{1 / 2}\right) \times H$ with inner product

$$
\left\langle\left(\begin{array}{l}
u \\
v
\end{array}\right),\left(\begin{array}{l}
u_{1} \\
v_{1}
\end{array}\right)\right\rangle_{\mathcal{H}}=\left(A^{1 / 2} u, A^{1 / 2} u_{1}\right)+\left(v, v_{1}\right) .
$$

Define the matrix operator $C: D(C) \rightarrow \mathcal{H}$ by

$$
D(C)=D(A) \times D\left(A^{1 / 2}\right), \quad C=\left(\begin{array}{cc}
0 & -I \\
A & 0
\end{array}\right) .
$$

For $\beta=\alpha / 2, h_{0}=\left(\begin{array}{l}0 \\ f\end{array}\right)$ consider the following problem in $\mathcal{H}$

$$
\begin{aligned}
& D_{t}^{\beta} w(t)+C w(t)=h_{0}(t), \quad \text { a.a. } t \in I, \\
& \left(g_{1-\beta} * w\right)(0)=0 .
\end{aligned}
$$

Proposition 5.7 Let $\alpha \in(1,2), \beta=\alpha / 2$. If for any $f \in L^{2}(I ; H)$ equation (5.30) has a unique solution $w \in R_{0}^{\beta, 2}(I, \mathcal{H}) \cap L^{2}(I ; D(C))$ then the problem (5.27) has maximal regularity in $L^{2}(I ; H)$.

Proof: Let $\mathcal{A}$ and $\mathcal{C}$ denote the extensions of $A$ and $C$ to $L^{2}(I ; H)$ and $L^{2}(I ; \mathcal{H})$, respectively. For $\alpha \in(0,2), \beta \in(0,1)$, define operators $\mathcal{L}_{\alpha}$ and ${ }_{\mathcal{H}} \mathcal{L}_{\beta}$ as follows

$$
\begin{gathered}
D\left(\mathcal{L}_{\alpha}\right)=R_{0}^{\alpha, 2}(I ; H), \quad \mathcal{L}_{\alpha} u=D_{t}^{\alpha} u, u \in D\left(\mathcal{L}_{\alpha}\right), \\
D\left({ }_{\mathcal{H}} \mathcal{L}_{\beta}\right)=R_{0}^{\beta, 2}(I ; \mathcal{H}), \quad{ }_{\mathcal{H}} \mathcal{L}_{\beta} u=D_{t}^{\beta} u, u \in D\left({ }_{\mathcal{H}} \mathcal{L}_{\beta}\right) .
\end{gathered}
$$

Problems (5.27) and (5.30) can be written in operator form as

$$
\begin{gathered}
\mathcal{L}_{\alpha} u+\mathcal{A} u=f ; \\
\mathcal{H}_{\beta} w+\mathcal{C} w=h_{0} .
\end{gathered}
$$

Let $w=\left(\begin{array}{c}u \\ v\end{array}\right)$ be the unique solution of (5.30). Therefore $v=\mathcal{L}_{\beta} u$ and $\mathcal{L}_{\beta} v+\mathcal{A} u=f$. Since $\mathcal{L}_{\beta}^{2}=\mathcal{L}_{\alpha}$, then $u$ satisfies (5.33). The maximal regularity estimate for $(5.30)$

$$
\left\|{ }_{\mathcal{H}} \mathcal{L}_{\beta} w\right\|_{L^{2}(I ; \mathcal{H})}+\|\mathcal{C} w\|_{L^{2}(I ; \mathcal{H})} \leq M\left\|h_{0}\right\|_{L^{2}(I ; \mathcal{H})}
$$


is equivalent to

$$
\left\|\mathcal{A}^{1 / 2} \mathcal{L}_{\beta} u\right\|_{L^{2}(I ; H)}+\left\|\mathcal{L}_{\beta} v\right\|_{L^{2}(I ; H)}+\left\|\mathcal{A}^{1 / 2} v\right\|_{L^{2}(I ; H)}+\|\mathcal{A} u\|_{L^{2}(I ; H)} \leq M_{1}\|f\|_{L^{2}(I ; H)},
$$

which by $\mathcal{L}_{\beta} v=\mathcal{L}_{\alpha} u$ implies

$$
\left\|\mathcal{L}_{\alpha} u\right\|_{L^{2}(I ; H)}+\|\mathcal{A} u\|_{L^{2}(I ; H)} \leq M_{1}\|f\|_{L^{2}(I ; H) \cdot} \cdot \square
$$

In fact a somewhat stronger result holds, showing that it is natural to expect that problems (5.27) and (5.30) simultaneously have or do not have maximal $L^{2}$ regularity. Namely, if $H$ is a complex Hilbert space then the following relation between the spectral angles of $A$ and $C$ holds

$$
2 \omega_{C}=\omega_{A}+\pi
$$

Indeed, suppose that $A$ is nonnegative with spectral angle $\omega_{A}$. By (5.43) we obtain for $\lambda \in \Sigma_{\frac{1}{2}\left(\pi-\omega_{A}-\varepsilon\right)}$

$|\lambda|^{2}\left\|(\lambda+C)^{-1} h\right\|_{\mathcal{H}}^{2}=\left\|\lambda A^{1 / 2}\left(\lambda^{2}+A\right)^{-1}(g+\lambda f)\right\|^{2}+\left\|\lambda^{2}\left(\lambda^{2}+A\right)^{-1} g-\lambda A^{1 / 2}\left(\lambda^{2}+A\right)^{-1} A^{1 / 2} f\right\|^{2}$.

Using the inequality $\|a+b\|^{2} \leq 2\left(\|a\|^{2}+\|b\|^{2}\right)$, we have

$$
\begin{aligned}
|\lambda|^{2}\left\|(\lambda+C)^{-1} h\right\|_{\mathcal{H}}^{2} & \leq 2\left\|\lambda A^{1 / 2}\left(\lambda^{2}+A\right)^{-1} g\right\|^{2}+2\left\|\lambda^{2} A^{1 / 2}\left(\lambda^{2}+A\right)^{-1} f\right\|^{2} \\
& +2\left\|\lambda^{2}\left(\lambda^{2}+A\right)^{-1} g\right\|^{2}+2\left\|\lambda A^{1 / 2}\left(\lambda^{2}+A\right)^{-1} A^{1 / 2} f\right\|^{2} .
\end{aligned}
$$

Since the spectral angle of $A$ is $\omega_{A}$ it follows for $\lambda \in \Sigma_{\frac{1}{2}\left(\pi-\omega_{A}-\varepsilon\right)}$,

$$
\left\|\lambda^{2}\left(\lambda^{2}+A\right)^{-1}\right\| \leq c_{\varepsilon}, \quad\left\|A\left(\lambda^{2}+A\right)^{-1}\right\| \leq c_{\varepsilon}+1 .
$$

Applying [69], Proposition 2.3.3, these two inequalities imply for such $\lambda$,

$$
\left\|\lambda A^{1 / 2}\left(\lambda^{2}+A\right)^{-1}\right\| \leq c_{\varepsilon}^{\prime} .
$$

Substituting the above inequalities in (5.39), we obtain that there exists a constant $M_{\varepsilon}$, such that for any $\lambda \in \Sigma_{\frac{1}{2}\left(\pi-\omega_{A}-\varepsilon\right)}$

$$
|\lambda|^{2}\left\|(\lambda+C)^{-1} h\right\|_{\mathcal{H}}^{2} \leq M_{\varepsilon}^{2}\left(\left\|A^{1 / 2} f\right\|^{2}+\|g\|^{2}\right)
$$

Therefore, the spectral angle $\omega_{C}$ of $C$ satisfies $\omega_{C} \leq \frac{\omega_{A}+\pi}{2}$. Conversely, if $C$ has spectral angle $\omega_{C}$ then (5.40) is satisfied for $\lambda \in \Sigma_{\pi-\omega_{C}-\varepsilon}$. Taking $f=0$ we obtain

$$
\left\|\lambda^{2}\left(\lambda^{2}+A\right)^{-1}\right\| \leq M_{\varepsilon}, \quad \lambda \in \Sigma_{\pi-\omega_{C}-\varepsilon} .
$$

Hence $A$ is nonnegative and $\omega_{A} \leq 2 \omega_{C}-\pi$. Therefore (5.38) holds. Since $\omega_{\mathcal{L}_{\alpha}}=$ $\alpha \pi / 2, \omega_{\mathcal{H}} \mathcal{L}_{\beta}=\beta \pi / 2($ Lemma $1.8(\mathrm{~d}))$ then (5.38) shows that the inequality $\omega_{\mathcal{L}_{\alpha}}+\omega_{\mathcal{A}}<\pi$ is equivalent to $\omega_{\mathcal{H}} \mathcal{L}_{\beta}+\omega_{\mathcal{C}}<\pi$ if $\beta=\alpha / 2$.

Now instead of (5.27) we would like to study (5.30) by the method of sums of accretive operators. Thus, we need $C$ to be $m$-accretive. Next lemma gives the relationship between the properties of operators $A$ and $C$. 
Lemma 5.8 Let $A$ be an $m$-accretive operator, $0 \in \rho(A)$. Then $C$ is $m$-accretive iff $A$ is self-adjoint.

Proof: If $A$ is an $m$-accretive and self-adjoint operator with $0 \in \rho(A)$ then $A^{1 / 2}$ has the same properties. If $w=\left(\begin{array}{l}u \\ v\end{array}\right) \in D(C)$, then

$$
\langle C w, w\rangle_{\mathcal{H}}=-\left(A^{1 / 2} v, A^{1 / 2} u\right)+(A u, v)
$$

and therefore $\langle C w, w\rangle_{\mathcal{H}}=0$. So, $C$ and $-C$ are $m$-accretive. Moreover, $R(\lambda I+A)=$ $H, \lambda>0$, implies $R(\lambda I+C)=\mathcal{H}, \lambda>0$. Indeed, consider the equation

$$
\lambda w+C w=h, \quad h \in \mathcal{H}, \lambda>0 .
$$

If $w=\left(\begin{array}{l}u \\ v\end{array}\right)$, and $h=\left(\begin{array}{l}f \\ g\end{array}\right)$, then for any $\lambda>0$ its solution is given by

$$
u=\left(\lambda^{2}+A\right)^{-1}(g+\lambda f), v=\lambda\left(\lambda^{2}+A\right)^{-1} g-A^{1 / 2}\left(\lambda^{2}+A\right)^{-1} A^{1 / 2} f .
$$

Since $f \in D\left(A^{1 / 2}\right), g \in H$, then $u \in D(A), v \in D\left(A^{1 / 2}\right)$, i.e. (5.42) has unique solution $w \in D(C)$ and thus $R(\lambda I+C)=\mathcal{H}, \lambda>0$. Therefore $C$ is $m$-accretive.

Conversely, let $C$ be $m$-accretive. This implies by (5.41) that $(A u, v) \geq\left(A^{1 / 2} u, A^{1 / 2} v\right)$. Setting $u_{1}=A^{1 / 2} u$, we have $\left(A^{1 / 2} u_{1}, v\right) \geq\left(u_{1}, A^{1 / 2} v\right)$ for all $u_{1}, v \in D\left(A^{1 / 2}\right)$. Taking now $-u_{1}$ instead of $u_{1}$, we obtain in fact equality

$$
\left(A^{1 / 2} u_{1}, v\right)=\left(u_{1}, A^{1 / 2} v\right), \quad \forall u_{1}, v \in D\left(A^{1 / 2}\right) .
$$

Therefore $A^{1 / 2}$ and thus also $A$ is symmetric. Since it is by assumption also $m$-accretive, the following basic lemma ( see [48] ) shows that it is self-adjoint.

Lemma 5.9 $A$ linear operator $A$ is self-adjoint and accretive, iff it is symmetric and m-accretive. If in addition $0 \in \rho(A)$, then there exists $\beta>0$ such that $(A u, u) \geq \beta\|u\|^{2}$.

Proof: Let $A$ be self-adjoint and accretive. Since $\lambda I+A$ is self-adjoint for any $\lambda>0$, then $H=N(\lambda I+A) \oplus R(\lambda I+A)[48]$. But $N(\lambda I+A)=\{0\}$ by the accretivity of $A$. Therefore $R(\lambda I+A)=H$ and $\mathrm{A}$ is m-accretive.

Let now $A$ be m-accretive and symmetric. Therefore $(I+A)^{-1}$ is bounded operator. Then [48], Ch. 3, Theorem 5.30, implies that $\left((I+A)^{*}\right)^{-1}$ is bounded, too, and $((I+$ $\left.A)^{*}\right)^{-1}=\left((I+A)^{-1}\right)^{*}$. Since $(I+A)^{-1}$ is bounded symmetric, it is self-adjoint. Therefore $\left((I+A)^{-1}\right)^{*}=(I+A)^{-1}$. Thus we obtain $(I+A)^{-1}=\left(I+A^{*}\right)^{-1}$, which implies that $D(A)=D\left(A^{*}\right)$ and $A u=A^{*} u$ for all $u \in D(A)$. Therefore $A$ is self-adjoint.

To prove the last part of the proposition we note that $\sigma(A) \subset(-\infty,+\infty)$ because $A$ is self-adjoint. Since it is accretive, we have $(-\infty, 0) \subset \rho(A)$. If moreover $0 \in \rho(A)$ then (since the resolvent set is open) there exists $\beta>0$ such that $(-\infty, \beta) \in \rho(A)$. Therefore 
the operator $A_{\beta}=A-\beta$ has its spectrum on the nonnegative real axis and for any $\lambda>0$, $\left\|\left(A_{\beta}+\lambda\right)^{-1}\right\|_{\mathcal{L}(H)} \leq \lambda^{-1}$. Hence, if $u \in D(A)$ we obtain in $H$ :

$$
\begin{gathered}
\|u\| \leq \lambda^{-1}\left\|\left(A_{\beta}+\lambda\right) u\right\| \\
\|u\|^{2} \leq \lambda^{-2}\left\|A_{\beta} u\right\|^{2}+2 \lambda^{-1}\left(A_{\beta} u, u\right)+\|u\|^{2} \\
0 \leq \lambda^{-1}\left\|A_{\beta} u\right\|^{2}+2\left(A_{\beta} u, u\right) .
\end{gathered}
$$

Taking $\lambda \rightarrow \infty$ we obtain $\left(A_{\beta} u, u\right) \geq 0$.

According to Lemma 5.8, $C$ is $m$-accretive only if $A$ is self-adjoint. So, our idea to reduce the problem of order $\alpha \in(1,2)$ to a problem of order $\alpha / 2$ and applying to it Theorem 5.5 works only for self-adjoint operators $A$. But in this case maximal regularity can be obtained by spectral theory and this is the most natural way.

\subsection{Linear nonautonomous problems with $\alpha \in(0,1)$}

In this section we suppose that $\alpha \in(0,1)$ and $I=(0, T)$, where $T>0$. Let $H$ be a complex Hilbert space and $f \in L^{2}(I ; H)$. Consider the linear nonautonomous problem

$$
\begin{aligned}
& D_{t}^{\alpha} u(t)+C(t) A u(t)=f(t), \quad \text { a.a. } t \in I, \\
& \left(g_{1-\alpha} * u\right)(0)=0 .
\end{aligned}
$$

where $A: D(A)=: H_{1} \rightarrow H$, where $H_{1}$ is densely embedded in $H$ and endowed with the norm $\|x\|_{H_{1}}=\|A x\|_{H}$, and moreover

(A) $A$ is accretive and self-adjoint in $H, 0 \in \rho(A)$;

(C) $C($.$) defines a bounded operator in L^{2}(I ; H)$ and there exists $\gamma>0$, such that $\operatorname{Re}(C(.) u, u)_{L^{2}(I ; H)} \geq \gamma\|u\|_{L^{2}(I ; H)}^{2}$ for any $u \in L^{2}(I ; H)$.

Note that Lemma 5.9 elucidates condition $(\boldsymbol{A})$.

Further, define operators $\mathcal{A}$ and $\mathcal{C}$ in $L^{2}(I ; H)$ as follows

$$
\begin{gathered}
D(\mathcal{A})=L^{2}\left(I ; H_{1}\right), \quad(\mathcal{A} u)(t)=A u(t), \text { for a.a. } t \in I, u \in D(\mathcal{A}) ; \\
D(\mathcal{C})=L^{2}(I ; H), \quad(\mathcal{C} u)(t)=C(t) u(t), \text { for a.a. } t \in I .
\end{gathered}
$$

Then equation (5.44) can be rewritten as an operator equation of the form

$$
\mathcal{L}_{\alpha} u+\mathcal{C} \mathcal{A} u=f
$$

or, setting $v=\mathcal{A}^{-1} u$, as

$$
\mathcal{L}_{\alpha} \mathcal{A}^{-1} v+\mathcal{C} v=f
$$

The operator $\mathcal{A}$ is $m$-accretive as an extension of the $m$-accretive operator $A$ ([30], Section 2.4). Moreover, $\mathcal{A}$ is symmetric and $0 \in \rho(\mathcal{A})$, because $A$ has these properties. Consider the operator $\mathcal{L}_{\alpha} \mathcal{A}^{-1}$ on $D\left(\mathcal{L}_{\alpha} \mathcal{A}^{-1}\right)=\left\{u \in L^{2}(I ; H) \mid \mathcal{A}^{-1} u \in D\left(\mathcal{L}_{\alpha}\right)\right\}$. Applying [13], 
Proposition 2.1, it follows that $\mathcal{L}_{\alpha} \mathcal{A}^{-1}$ is $m$-accretive. Concerning $\mathcal{C}-\gamma$, it is immediate by $(\boldsymbol{C})$, that it is a bounded accretive operator. Therefore, a natural way to prove solvability of (5.46), resp (5.45), is to apply a specialization of Lemma 5.4 with $\mathcal{B}$ an accretive bounded operator. According to this lemma $\mathcal{L}_{\alpha} \mathcal{A}^{-1}+\mathcal{C}-\gamma$ is $m$-accretive, that is, for any $f \in L^{2}(I ; H)$ the equation (5.46) has a unique solution $v \in L^{2}(I ; H)$. By taking the inner product of (5.46) with $v$ we obtain by using the accretivity of $\mathcal{L}_{\alpha} \mathcal{A}^{-1}$ and $\mathcal{C}-\gamma$ and Cauchy-Schwarz inequality that

$$
\|v\|_{L^{2}(I ; H)} \leq \frac{1}{\gamma}\|f\|_{L^{2}(I ; H)} .
$$

Further, from the boundedness of $\mathcal{C},(5.46)$ and $(5.47)$ we obtain that there exists a constant $C$ such that

$$
\left\|\mathcal{L}_{\alpha} \mathcal{A}^{-1} v\right\|_{L^{2}(I ; H)}+\|v\|_{L^{2}(I ; H)} \leq C\|f\|_{L^{2}(I ; H)} .
$$

Therefore $u=\mathcal{A}^{-1} v$ is the unique solution of (5.45), for which (5.48) implies

$$
\left\|\mathcal{L}_{\alpha} u\right\|_{L^{2}(I ; H)}+\|\mathcal{A} u\|_{L^{2}(I ; H)} \leq C\|f\|_{L^{2}(I ; H)} .
$$

So, we proved the following result:

Theorem 5.10 Let $\alpha \in(0,1), H$ be a complex Hilbert space and suppose that $(\boldsymbol{A})$ and $(C)$ hold. Then for any $f \in L^{2}(I ; H)$ there exists a unique function

$$
u \in R_{0}^{\alpha, 2}(I ; H) \cap L^{2}\left(I ; H_{1}\right),
$$

satisfying (5.44) for which the following estimate holds

$$
\|u\|_{L^{2}(I ; H)}+\left\|D_{t}^{\alpha} u\right\|_{L^{2}(I ; H)}+\|u\|_{L^{2}\left(I ; H_{1}\right)} \leq C\|f\|_{L^{2}(I ; H)} .
$$

Since for Hilbert spaces $H, R_{0}^{\alpha, p}(I ; H)=H_{0}^{\alpha, p}(I ; H), \alpha \neq 1 / 2$, in this case we can reformulate the above result in terms of Bessel potential spaces $H_{0}^{\alpha, p}(I ; H)$.

In order to apply Lemma 5.4 we needed the fact that $\mathcal{L}_{\alpha} \mathcal{A}^{-1}$ is $m$-accretive. It is interesting to see to what extent the self-adjointness of $A$ in condition $(\boldsymbol{A})$ could be relaxed, preserving the $m$-accretivity of $\mathcal{L}_{\alpha} \mathcal{A}^{-1}$. First note that condition $R\left(\lambda I+\mathcal{L}_{\alpha} \mathcal{A}^{-1}\right)=\mathcal{H}$, $\lambda>0$ is satisfied for any $m$-accretive $\mathcal{A}$, because in this case the equation $\lambda \mathcal{A} u+\mathcal{L}_{\alpha} u=f$ is uniquely solvable for any $\lambda>0, f \in L^{2}(I ; H)$ (Theorem 5.5, which holds also for complex Hilbert space $H$ ). The accretivity of $\mathcal{L}_{\alpha} \mathcal{A}^{-1}$ :

$$
\operatorname{Re}\left(\mathcal{L}_{\alpha} \mathcal{A}^{-1} v, v\right)_{L^{2}(I ; H)} \geq 0, v \in D\left(\mathcal{L}_{\alpha} \mathcal{A}^{-1}\right),
$$

is equivalent to $\operatorname{Re}\left(u, \mathcal{J}_{\alpha} \mathcal{A} u\right)_{L^{2}(I ; H)} \geq 0, u \in D(\mathcal{A})$. This holds whenever $g_{\alpha}(t) A$ is an operator-valued kernel of positive type [64], that is

$$
\operatorname{Re} \int_{0}^{T}\left(\int_{0}^{t} g_{\alpha}(t-\tau) A u(\tau) d \tau, u(t)\right)_{H} d t \geq 0, \quad u \in L^{2}\left(I ; H_{1}\right) .
$$

The following lemma ([64], Proposition 6.7) gives necessary and sufficient conditions for a kernel $k(t) A$ to be of positive type. 
Lemma 5.11 Let $k(t) \in L^{1}(I ; \mathbb{R})$ and $A \in \mathcal{B}\left(H_{1}, H\right)$. Then $k(t) A$ is a kernel of positive type if and only if

$$
\operatorname{Re}\left[\widehat{k}(\lambda)(A u, u)_{H}\right] \geq 0, \quad \forall u \in H_{1}, \lambda \in \Sigma_{\pi / 2},
$$

where $\widehat{k}$ is the Laplace transform of $k$.

Since $\widehat{g_{\alpha}}(\lambda)=\lambda^{-\alpha}$, the inequality (5.51) is satisfied for $k=g_{\alpha}, \alpha \in(0,1)$, and for all $\lambda \in \Sigma_{\pi / 2}$, if and only if

$$
\left|\arg (A u, u)_{H}\right| \leq(1-\alpha) \pi / 2 .
$$

Note that this property is stronger than accretivity only $\left((A u, u)_{H} \subseteq \Sigma_{\pi / 2}\right)$ and weaker than accretivity + self-adjointness $\left((A u, u)_{H} \subseteq \mathbb{R}_{+}\right)$. Inequality (5.52) is equivalent to

$$
(\tan \alpha \pi / 2)\left|\operatorname{Im}(A u, u)_{H}\right| \leq \operatorname{Re}(A u, u)_{H}, u \in H_{1},
$$

when $\alpha \in(0,1)$. An $m$-accretive operator, satisfying such an inequality is called regularly $\boldsymbol{m}$-accretive (see [64], p.233). Therefore we can relax condition $(\boldsymbol{A})$ to

$\left(\boldsymbol{A}^{\prime}\right) A$ is regularly $m$-accretive with angle $\alpha \pi / 2$ (i.e. (5.52), resp (5.53) is satisfied).

Since Lemma 5.4 holds for nonlinear, accretive, Lipschitz continuous everywhere defined operators $\mathcal{B}$, we can make further generalizations of Theorem 5.10 assuming instead of $(C)$ the following condition

$\left(\boldsymbol{C}^{\prime}\right) C(t)$ is nonlinear operator, everywhere defined in $L^{2}(I ; H)$, satisfying for any $u_{1}, u_{2} \in$ $L^{2}(I ; H)$

$$
\begin{gathered}
\left\|C(t) u_{1}-C(t) u_{2}\right\|_{L^{2}(I ; H)} \leq M\left\|u_{1}-u_{2}\right\|_{L^{2}(I ; H)}, \\
\left(C(t) u_{1}-C(t) u_{2}, u_{1}-u_{2}\right)_{L^{2}(I ; H)} \geq \gamma\left\|u_{1}-u_{2}\right\|_{L^{2}(I ; H)}^{2}
\end{gathered}
$$

In this way we obtain

Theorem 5.12 Let $\alpha \in(0,1)$ and suppose that $\left(\boldsymbol{A}^{\prime}\right)$ and $\left(\boldsymbol{C}^{\prime}\right)$ are satisfied. Then the claims of Theorem 5.10 hold.

Another generalization of Theorem 5.10 is to consider instead of (5.44) a more general integrodifferential equation

$$
\frac{d}{d t}(k * u)+C(t) A u=f(t), \text { a.a. } t \in I
$$

where $k \in L^{1}(I ; \mathbb{R})$ is nonnegative and nonincreasing. To study this problem, consider the class of operators, defined as follows:

$$
D(K)=\left\{u \in L^{p}(I) \mid k * u \in W_{0}^{1, p}(I)\right\}, K u=\frac{d}{d t}(k * u), u \in D(K),
$$

where $k \in L^{1}(I ; \mathbb{R})$ is nonnegative and nonincreasing. Next result is proven in [19], Th.3.1. Actually, [19] deals with $L^{p}(I ; \mathbb{R})$, however, without any changes the proof carries over to $L^{p}(I ; \mathbb{C})$. 
Lemma 5.13 Let the operator $K$ be defined by (5.54). Then $K$ is m-accretive operator in $L^{p}(I ; \mathbb{C})$ and $0 \in \rho(K)$.

Suppose moreover that $k$ satisfies $|\arg \widehat{k}(\lambda)| \leq \varphi_{k}<\pi / 2$ for all $\lambda \in \Sigma_{\pi / 2}$. Then the condition $\left(\boldsymbol{A}^{\prime}\right)$ has to be suitably changed as follows

$\left(\boldsymbol{A}_{\boldsymbol{k}}^{\prime}\right) A$ is regularly $m$-accretive, satisfying

$$
\left(\tan \varphi_{k}\right)\left|\operatorname{Im}(A u, u)_{H}\right| \leq \operatorname{Re}(A u, u)_{H}, u \in H_{1} .
$$

Define the operators $\mathcal{K}$ by

$$
D(\mathcal{K})=\left\{u \in L^{p}(I ; X) \mid k * u \in W_{0}^{1, p}(I ; X)\right\}, \mathcal{K} u=\frac{d}{d t}(k * u), u \in D(\mathcal{K}),
$$

Then $\mathcal{K}$ is the $m$-accretive extension of $K$ to $L^{p}(0, T ; X)$, therefore the properties given in Lemma 5.13 remains true for $\mathcal{K}$ in $L^{p}(0, T ; X)$. According to Lemma $5.11, \mathcal{K} \mathcal{A}^{-1}$ is $m$-accretive iff $A$ satisfies $\left(\boldsymbol{A}_{\boldsymbol{k}}^{\prime}\right)$ and the rest follows from Lemma 5.4. Thus, we obtained

Theorem 5.14 Suppose that $k \in L^{1}(I ; \mathbb{R})$ is nonnegative and nonincreasing, satisfying $|\arg \widehat{k}(\lambda)| \leq \varphi_{k}<\pi / 2$ for all $\lambda \in \Sigma_{\pi / 2}$ and that $\left(\boldsymbol{A}_{\boldsymbol{k}}^{\prime}\right)$ and $(\boldsymbol{C})$ (or $\left(\boldsymbol{C}^{\prime}\right)$ ) hold. Then for any $f \in L^{2}(I ; H)$ there exists a unique function

$$
u \in D(\mathcal{K}) \cap L^{2}\left(I ; H_{1}\right),
$$

satisfying

$$
\frac{d}{d t}(k * u)(t)+C(t) A u(t)=f(t), \quad \text { a.a. } t \in I,
$$

for which the following estimate holds

$$
\|u\|_{L^{2}(I ; H)}+\left\|\frac{d}{d t}(k * u)\right\|_{L^{2}(I ; H)}+\|u\|_{L^{2}\left(I ; H_{1}\right)} \leq C\|f\|_{L^{2}(I ; H)} .
$$

In spite of these generalizations, the conditions required are still quite restrictive. It is known (see e.g. [64], p. 233) that an operator $A$ satisfying $\left(\boldsymbol{A}^{\prime}\right)$ belongs to $\mathcal{B I} \mathcal{P}(H ; \theta)$ with $\theta \leq(1-\alpha) \pi / 2$. If we take for example $C(t)=C$, a positive constant, we know that (5.44) is solvable under much weaker condition $\theta<(2-\alpha) \pi / 2$ (Theorem 5.2). Another disadvantage of this method is that we can not study (5.44) with $\alpha \in(1,2)$, because in this case $\mathcal{L}_{\alpha}$ are not $m$-accretive. Anyway, it is a simple method, which can be used in applications. An example is considered in the next section.

\subsection{Example: fractional Löwner-Kufarev equation}

For a recent study on the linear and quasilinear Löwner-Kufarev equation we refer to [51], [40], [41], see also the references therein. Let $D:=\{z \in \mathbb{C}:|z|<1\}$ be the unit disk, $T>0$. Recall the linear Löwner-Kufarev equation:

$$
\Omega_{t}(z, t)=z F(z, t) \Omega_{z}(z, t), \Omega(z, 0)=\Omega_{0}(z),
$$


where $F(z, t)$ is a continuous function on $\bar{D} \times I$ and analytic with respect to $z \in D$ for all fixed $t \in I$, and $\Omega_{0}(z)$ is an analytic function on $D$. Its study is important as a transient case to the quasilinear Löwner-Kufarev equation.

We are concerned with the "fractional approximation" of the problem (5.59), where the first derivative on time is replaced by the Riemann-Liouville fractional derivative of order $\alpha \in(0,1)$, namely

$$
D_{t}^{\alpha} \Omega(z, t)=z F(z, t) \Omega_{z}(z, t),\left(g_{1-\alpha} * \Omega(z, .)\right)(0)=\Omega_{0}(z), \quad \alpha \in(0,1),
$$

and call it fractional Löwner-Kufarev equation

It turns out that if we want to discuss the existence of solutions, it is more appropriate to consider time dependent functions in the Hilbert space $H:=L^{2,+}(0,2 \pi ; \mathbb{C})$, consisting of all functions of the form $f(\theta)=\sum_{n=1}^{\infty} f_{n} e^{i n \theta}$, for which $\sum_{n=1}^{\infty}\left|f_{n}\right|^{2}<\infty$, and endowed with the inner product

$$
(f, g)=\frac{1}{2 \pi} \int_{0}^{2 \pi} f(\theta) \overline{g(\theta)} d \theta=\sum_{n=0}^{\infty} f_{n} \bar{g}_{n}
$$

Denote

$$
W^{1,2,+}(0,2 \pi ; \mathbb{C}):=\left\{f \in L^{2,+}(0,2 \pi ; \mathbb{C}): \sum_{n=1}^{\infty} n^{2}\left|f_{n}\right|^{2}<\infty\right\} .
$$

Define an operator $A$ by

$$
D(A):=W^{1,2,+}(0,2 \pi ; \mathbb{C}), \quad \text { Af }(\theta):=-i f^{\prime}(\theta)=\sum_{n=1}^{\infty} n f_{n} e^{i n \theta}
$$

This operator yields the following properties:

$A$ is accretive and self-adjoint, $0 \in \rho(A)$.

Indeed, we have $(A f, f)=\sum_{n=1}^{\infty} n\left|f_{n}\right|^{2}>0$ and $(A f, g)=\sum_{n=1}^{\infty} n f_{n} \overline{g_{n}}=(f, A g)$, so that $A$ is accretive and symmetric. According to Lemma 5.9 it remains to show that $R(\lambda I+A)=H, \lambda \geq 0$. But it is immediate that for any $\lambda \geq 0$ and $g \in H$ the equation $\lambda f+A f=g$ has a unique solution with $f_{n}=(\lambda+n)^{-1} g_{n}$.

Let $H_{1}:=D(A)=W^{1,2,+}(0,2 \pi ; \mathbb{C})$ with $(f, g)_{H_{1}}:=(A f, A g)_{H}$. Since $A$ satisfies $(5.62)$ then $A^{1 / 2}$ exists and has the same properties. Its explicit representation reads

$$
D\left(A^{1 / 2}\right)=\left\{f \in L^{2,+}(0,2 \pi ; \mathbb{C}): \sum_{n=1}^{\infty} \sqrt{n}\left|f_{n}\right|^{2}<\infty\right\}, \quad A^{1 / 2} f(\theta)=\sum_{n=1}^{\infty} \sqrt{n} f_{n} e^{i n \theta}
$$

Define

$$
\begin{aligned}
& H_{1 / 2}:=D\left(A^{1 / 2}\right), \quad(f, g)_{H_{1 / 2}}:=\left(A^{1 / 2} f, A^{1 / 2} g\right)_{H} ; \\
& H_{3 / 2}:=D\left(A^{3 / 2}\right)=\left\{u \in H_{1}: A u \in H_{1 / 2}\right\}, \quad(f, g)_{H_{3 / 2}}:=\left(A^{3 / 2} f, A^{3 / 2} g\right)_{H} .
\end{aligned}
$$


Let the family of operators $\{C(t)\}_{t \in I}$ be defined by

$$
C(t) u(\theta, t)=-F\left(e^{i \theta}, t\right) u(\theta, t), \quad \theta \in[0,2 \pi], t \in I .
$$

Since $F\left(e^{i \theta}, t\right)$ is a continuous function on $(\theta, t) \in[0,2 \pi] \times I$ then there exists a constant $M>0$ such that $\left|F\left(e^{i \theta}, t\right)\right| \leq M,(\theta, t) \in[0,2 \pi] \times I$. Therefore

$$
C(.) \in \mathcal{B}\left(L^{2}(I ; H)\right)
$$

If we suppose moreover

$$
\operatorname{Re} F\left(e^{i \theta}, t\right) \leq-\gamma<0
$$

then for any $u \in L^{2}(I ; H)$

$$
\operatorname{Re}(C(.) u, u)_{L^{2}(I ; H)}=\frac{-1}{2 \pi} \operatorname{Re} \int_{0}^{T} \int_{0}^{2 \pi} F\left(e^{i \theta}, t\right)|u(\theta, t)|^{2} d \theta d t \geq \gamma\|u\|_{L^{2}(I ; H)}^{2} .
$$

So, the operator $C(t)$ is strictly accretive in $L^{2}(I ; H)$, i.e. there exists $\gamma>0$ such that

$$
\operatorname{Re}(C(.) u, u)_{L^{2}(I ; H)} \geq \gamma\|u\|_{L^{2}(I ; H)}^{2}, \forall u \in L^{2}(I ; H) .
$$

On the Hilbert space $H_{1 / 2}$ consider the family of operators $\{A(t)\}_{t \in I}$, defined by

$$
D(A(t)):=H_{3 / 2}, \quad A(t):=C(t) A .
$$

Again by the continuity of $F$, it follows $A(.) \in C\left(I ; \mathcal{B}\left(H_{3 / 2}, H_{1 / 2}\right)\right)$. If (5.66) is satisfied then the extension of the operator $A(s)\left(s \in I\right.$ is fixed ) to $L^{2}\left(I ; H_{1 / 2}\right)$ is an $m$-accretive operator. Indeed, according to (5.67), if $s \in I$ is fixed then

$$
\operatorname{Re}(A(s) u, u)_{L^{2}\left(I ; H_{1 / 2}\right)}=\operatorname{Re}(C(s) A u, A u)_{L^{2}(I ; H)} \geq 0 .
$$

The fact that $R(\lambda I+A(s))=L^{2}\left(I ; H_{1 / 2}\right), \lambda \geq 0$, can be proven by solving the corresponding ordinary differential equation. The $m$-accretivity shows that $\omega_{A(s)} \leq \pi / 2$ and so $\omega_{A(s)}<\pi(1-\alpha / 2)$ for any $\alpha \in(0,1), s \in I$.

Some of the above properties will be used here and the rest in the next chapter to study maximal regularity of the fractional Löwner-Kufarev equation (5.60).

Substituting

$$
v(\theta, t):=\Omega\left(e^{i \theta}, t\right)-\Omega_{0}\left(e^{i \theta}\right) g_{\alpha}(t), \quad f(\theta, t):=e^{i \theta} F\left(e^{i \theta}, t\right) \Omega_{0}^{\prime}\left(e^{i \theta}\right) g_{\alpha}(t),
$$

we rewrite $(5.60)$ in the form

$$
D_{t}^{\alpha} v(t)+C(t) A v(t)=f(t),\left(g_{1-\alpha} * v\right)(0)=0 ; \quad \alpha \in(0,1) .
$$

Applying Theorem 5.10, the following result holds:

Proposition 5.15 Let $\alpha \in(0,1), H=L^{2,+}(0,2 \pi ; \mathbb{C}), H_{1}=W^{1,2,+}(0,2 \pi ; \mathbb{C})$. Assume $F\left(e^{i \theta}, t\right)$ is continuous on $(\theta, t) \in[0,2 \pi] \times I$ and (5.66) is satisfied. Let moreover $g_{\alpha}(t) F\left(e^{i \theta}, t\right) \in L^{2}(I ; H)$ and $\Omega_{0}\left(e^{i \theta}\right) \in H_{1}$. Then (5.60) has a unique solution

$$
\Omega\left(e^{i \theta}, t\right) \in L^{2}\left(I ; H_{1}\right) \cap H_{0}^{\alpha, p}(I ; H),
$$

satisfying

$$
\begin{gathered}
\|\Omega\|_{L^{2}(I ; H)}+\|\Omega\|_{H^{\alpha, p}(I ; H)}+\|\Omega\|_{L^{2}\left(I ; H_{1}\right)} \\
\leq C\left\|g_{\alpha}(t) F\left(e^{i \theta}, t\right)\right\|_{L^{2}(I ; H)}\left\|\Omega_{0}\right\|_{H_{1}}+c_{\alpha}(T)\left(\left\|\Omega_{0}\right\|_{H}+\left\|\Omega_{0}\right\|_{H_{1}}\right) .
\end{gathered}
$$




\section{Chapter 6}

\section{Quasilinear problems}

The goal of this last chapter is to develop a method to study fractional quasilinear problems of the form

$$
D_{t}^{\alpha} u+A(u) u=f
$$

where $A(u)$ is a linear operator for any fixed $u$. A natural way of solving such problems is by applying a fixed point argument to the equation

$$
D_{t}^{\alpha} u+A(v) u=f
$$

with $v$ given, which is a nonautonomous linear problem. For this reason we study first the solvability and maximal $L^{p}$ regularity of the nonautonomous problem for the fractional differential equation with the Riemann-Liouville fractional derivative of order $\alpha \in(0,2)$. To this aim we use the results on the strict $L^{p}$ solvability of the corresponding autonomous problems (Chapter 4) and work inductively in time. Next we establish the global existence of a strong solution of a quasilinear equation, intermediate to the quasilinear diffusion and wave equations.

\subsection{Linear nonautonomous case}

In this section we assume that $\alpha \in(0,2), 1<p<\infty, T>0$ and $X$ is a Banach space. Let $\{A(t)\}_{t \in[0, T]}$ be a family of linear closed densely defined operators on $X$, such that the domain of $A(t)$ does not depend on $t: D(A(t))=D(A(0))=: X_{1}$ and $0 \in \rho(A(t))$. We equip $X_{1}$ with the graph norm $\|x\|_{X_{1}}:=\|A x\|_{X}$.

If $\{A(t)\}_{t \in[0, T]}$ yields the above properties, then by the closed graph theorem, the operator $A(t) A(0)^{-1}$ is in $\mathcal{B}(X)$. Therefore, we have the representation $A(t)=C(t) A$, where $A=A(0)$ is closed linear operator in $X$ and $C(t)=A(t) A^{-1} \in \mathcal{B}(X)$. We used this factorization in Section 5.4.

Consider the problem

$$
D_{t}^{\alpha} u(t)+A(t) u(t)=f(t), \text { a.a. } t \in[0, T]
$$




$$
\begin{array}{ll}
\left(g_{1-\alpha} * u\right)(0)=x_{0}, & \text { if } \alpha \in(0,1), \\
\left(g_{2-\alpha} * u\right)(0)=x_{0},\left(g_{2-\alpha} * u\right)^{\prime}(0)=x_{1}, & \text { if } \alpha \in(1,2),
\end{array}
$$

where the forcing function $f(t) \in L^{p}(0, T ; X)$ and the initial data $x_{0}, x_{1} \in X$. In analogy with the autonomous case we give the following definition.

Definition 6.1 A function $u:[0, T] \rightarrow X$ is called a strict $\boldsymbol{L}^{p}$ solution of (6.1) on $[0, T]$ in $X$ if $u \in L^{p}\left(0, T ; X_{1}\right) \cap R^{\alpha, p}(0, T ; X)$ and (6.1), (6.2) are satisfied.

We suppose that the corresponding autonomous problems with $A=A(s)$, where $s \in[0, T]$ is fixed, are strictly solvable in $L^{p}(0, T ; X)$ with estimates, uniform on $s \in[0, T]$. On the base of these assumptions we are able to solve (6.1) inductively, dividing the interval $[0, T]$ in sufficiently small intervals, and to prove its strict $L^{p}$ solvability. This approach was introduced in [26] in the case $\alpha=1$ and it is used recently in [17], [42] for studying of some fractional equations.

Strictly, we suppose the following three properties of $A(t)$ :

(A1) $D(A(t))=D(A(0))=: X_{1}$; for any $t \in[0, T], A(t)$ is positive with spectral angle $\omega_{A(t)}<(1-\alpha / 2) \pi$ and $A(.) \in C\left(0, T ; \mathcal{B}\left(X_{1}, X\right)\right)$.

It follows from $(\boldsymbol{A 1})$ and the compactness of $[0, T]$ that the graph norms of the operators $A(t)$ are uniformly equivalent, i.e. there exist constants $a_{1}$ and $a_{2}$ such that for each $x \in X_{1}$ and $t \in[0, T]$ we have

$$
a_{1}\|x\|_{X_{1}} \leq\|A(t) x\|_{X} \leq a_{2}\|x\|_{X_{1}} .
$$

Denote by $\rho_{A, T}(s)$ the modulus of continuity of the continuous function $A(t)$, that is

$$
\rho_{A, T}(s):=\sup _{t_{1}, t_{2} \in[0, T],\left|t_{1}-t_{2}\right| \leq s}\left\|A\left(t_{1}\right)-A\left(t_{2}\right)\right\|_{\mathcal{B}\left(X_{1}, X\right)} .
$$

(A2) There exist subspaces $Z_{0}, Z_{1} \hookrightarrow X$ such that for any $x_{i} \in Z_{i}, i=0,1, f \in$ $L^{p}(0, T ; X)$ and for any fixed $s \in[0, T]$ the problem

$$
D_{t}^{\alpha} u(t)+A(s) u(t)=f(t), \quad \text { a.a. } t \in[0, T],
$$

with initial conditions (6.2) has a strict $L^{p}$ solution $u(t)$, satisfying the estimate (if $\alpha \in$ $(0,1)$ we set $\left.x_{1} \equiv 0\right)$ :

$$
\|u\|_{L^{p}\left(0, T ; X_{1}\right)} \leq M\left(\|f\|_{L^{p}(0, T ; X)}+\left\|x_{0}\right\|_{Z_{0}}+\left\|x_{1}\right\|_{Z_{1}}\right) .
$$

We suppose in addition

(A3) estimates (6.5) are uniform with respect to $s \in[0, T]$, i.e. the constant $M$ does not change for different values of $s \in[0, T]$.

Under these assumptions we have the following result on strict $L^{p}$ solvability of the nonautonomous problem (6.1), (6.2): 
Lemma 6.2 Let $\alpha \in(0,2)$ and assume that $(\boldsymbol{A 1}),(\boldsymbol{A 2})$ and $(\boldsymbol{A 3})$ hold. Then for any $x_{i} \in Z_{i}, i=0,1, f \in L^{p}(0, T ; X)$, there exists a unique strict $L^{p}$ solution of (6.1), (6.2), and the following estimate holds

$$
\|u\|_{L^{p}\left(0, T ; X_{1}\right)} \leq N\left(\|f\|_{L^{p}(0, T ; X)}+\left\|x_{0}\right\|_{Z_{0}}+\left\|x_{1}\right\|_{Z_{1}}\right),
$$

where $N$ depends only on $M$.

Proof: Let us note that (6.5) holds with $T$ replaced by an arbitrary $\hat{T} \in[0, T]$ and $M$ unchanged. To see this, consider the equation

$$
D_{t}^{\alpha} u(t)+A(s) u(t)=f(t), \text { a.a. } t \in[0, \hat{T}],
$$

where $f \in L^{p}(0, \hat{T} ; X)$ and define $f_{0}(t)=f(t)$, a.a. $t \in[0, \hat{T}]$ and $f_{0}(t)=0$, a.a. $t \in[\hat{T}, T]$. If $u_{0}(t)$ is the unique solution of (6.4) with $f$ replaced by $f_{0}$, then $u(t)=u_{0}(t)$, a.a. $t \in[0, \hat{T}]$, is the unique solution of $(6.7)$ and by the definition of $L^{p}$ norms we conclude that we in fact have our claim:

$$
\|u\|_{L^{p}\left(0, \hat{T} ; X_{1}\right)} \leq M\left(\|f\|_{L^{p}(0, \hat{T} ; X)}+\left\|x_{0}\right\|_{Z_{0}}+\left\|x_{1}\right\|_{Z_{1}}\right) .
$$

Take $T_{\varepsilon}$ such that

$$
\rho_{A, T}\left(T_{\varepsilon}\right) \leq \varepsilon:=\frac{a_{1}}{2 M a_{2}} .
$$

We solve first (6.1), (6.2) for $t \in\left[0, T_{\varepsilon}\right]$. For $v \in V_{\varepsilon}=L^{p}\left(0, T_{\varepsilon} ; X\right)$ we consider the equation

$$
D_{t}^{\alpha} u(t)+A(0) u(t)=f(t)+(A(0)-A(t)) A(0)^{-1} v(t)
$$

with initial conditions (6.2). Since the right-hand side is an element of $V_{\varepsilon}$ we obtain by the strong solvability of (6.10) that there is a unique solution $u \in V_{\varepsilon}$ such that $A(0) u \in V_{\varepsilon}$. Therefore the mapping $\gamma: v \rightarrow A(0) u$ maps $V_{\varepsilon}$ into itself and from (6.8) we have

$$
\begin{gathered}
\left\|\gamma\left(v_{1}\right)-\gamma\left(v_{2}\right)\right\|_{L^{p}\left(0, T_{\varepsilon} ; X\right)} \leq M\left\|(A(0)-A(t)) A(0)^{-1}\left(v_{1}-v_{2}\right)\right\|_{L^{p}\left(0, T_{\varepsilon} ; X\right)} \\
\leq M \sup _{t \in\left[0, T_{\varepsilon}\right]}\|A(0)-A(t)\|_{\mathcal{B}\left(X_{1}, X\right)}\left\|v_{1}-v_{2}\right\|_{L^{p}\left(0, T_{\varepsilon} ; X\right)} \leq M \rho_{A, T}\left(T_{\varepsilon}\right)\left\|v_{1}-v_{2}\right\|_{L^{p}\left(0, T_{\varepsilon} ; X\right)}
\end{gathered}
$$

According to (6.9) and noting that $a_{1} / a_{2} \leq 1$, the mapping $\gamma$ is a contraction and there is a unique fixed point. Thus we get a solution on the interval $\left[0, T_{\varepsilon}\right]$. Again by (6.8) and $(6.9)$

$$
\begin{gathered}
\|A(0) u\|_{L^{p}\left(0, T_{\varepsilon} ; X\right)} \leq M\left(\left\|f+(A(0)-A(t)) A(0)^{-1} v\right\|_{L^{p}\left(0, T_{\varepsilon} ; X\right)}+\left\|x_{0}\right\|_{Z_{0}}+\left\|x_{1}\right\|_{Z_{1}}\right) \\
\leq M\left(\|f\|_{L^{p}\left(0, T_{\varepsilon} ; X\right)}+\rho_{A, T}\left(T_{\varepsilon}\right)\|v\|_{L^{p}\left(0, T_{\varepsilon} ; X\right)}+\left\|x_{0}\right\|_{Z_{0}}+\left\|x_{1}\right\|_{Z_{1}}\right) \\
\leq M\left(\|f\|_{L^{p}(0, T ; X)}+\left\|x_{0}\right\|_{Z_{0}}+\left\|x_{1}\right\|_{Z_{1}}\right)+2^{-1}\|v\|_{L^{p}\left(0, T_{\varepsilon} ; X\right)} .
\end{gathered}
$$

Since $A(0) u=v$, then

$$
\|u\|_{L^{p}\left(0, T_{\varepsilon} ; X_{1}\right)} \leq 2 M\left(\|f\|_{L^{p}(0, T ; X)}+\left\|x_{0}\right\|_{Z_{0}}+\left\|x_{1}\right\|_{Z_{1}}\right) .
$$


Suppose now that $T_{0} \in[0, T]$ and that we have found a solution of (6.1), (6.2) on $\left[0, T_{0}\right]$ which satisfies the inequality

$$
\|u\|_{L^{p}\left(0, T_{0} ; X_{1}\right)} \leq N\left(T_{0}\right)\left(\|f\|_{L^{p}(0, T ; X)}+\left\|x_{0}\right\|_{Z_{0}}+\left\|x_{1}\right\|_{Z_{1}}\right) .
$$

Let $\hat{T}=\min \left\{T, T_{0}+T_{\varepsilon}\right\}$ and define the set

$$
V=\left\{v \in L^{p}(0, \hat{T} ; X) \mid v(t)=A\left(T_{0}\right) u(t), \text { a.a. } t \in\left[0, T_{0}\right]\right\} .
$$

For each $v \in V$ we proceed to find a solution $w$ of the equation

$$
D_{t}^{\alpha} w(t)+A\left(T_{0}\right) w(t)=f(t)+\left(A\left(T_{0}\right)-A(t)\right) A\left(T_{0}\right)^{-1} v(t)
$$

with initial conditions (6.2). Since the right-hand side of (6.12) is an element of $L^{p}(0, \hat{T} ; X)$, from the strong solvability of (6.12) there is a unique solution $w \in L^{p}(0, \hat{T} ; X)$ such that $A\left(T_{0}\right) w \in L^{p}(0, \hat{T} ; X)$ and the uniqueness guarantees $A\left(T_{0}\right) w \in V$. Denote the mapping $v \rightarrow A\left(T_{0}\right) w$ by $A\left(T_{0}\right) w=: G v$. By the linearity of (6.12) and applying (6.8) and (6.3) we obtain

$$
\left\|G\left(v_{1}\right)-G\left(v_{2}\right)\right\|_{L^{p}(0, \hat{T} ; X)} \leq a_{2} M\left\|\left(A\left(T_{0}\right)-A(t)\right) A\left(T_{0}\right)^{-1}\left(v_{1}-v_{2}\right)\right\|_{L^{p}(0, \hat{T} ; X)} .
$$

Since $v_{1}, v_{2} \in V$ then $v_{1}-v_{2}=0$ a.e. on $\left[0, T_{0}\right]$ and the last inequality implies

$$
\begin{gathered}
\left\|G\left(v_{1}\right)-G\left(v_{2}\right)\right\|_{L^{p}(0, \hat{T} ; X)} \leq a_{2} a_{1}^{-1} M \sup _{t \in\left[T_{0}, \hat{T}\right]}\left\|A\left(T_{0}\right)-A(t)\right\|_{\mathcal{B}\left(X_{1}, X\right)}\left\|v_{1}-v_{2}\right\|_{L^{p}(0, \hat{T} ; X)} \\
\leq a_{2} a_{1}^{-1} M \rho_{A, T}\left(T_{\varepsilon}\right)\left\|v_{1}-v_{2}\right\|_{L^{p}(0, \hat{T} ; X)} .
\end{gathered}
$$

Hence, by (6.9) the mapping $G$ is a contraction and thus we get a solution on the interval $[0, \hat{T}]$. If we take $v_{0} \in V$ to be such that $v_{0}(t)=0$ a.e. on $\left[T_{0}, \hat{T}\right]$, then $\left\|v_{0}\right\|_{L^{p}(0, \hat{T} ; X)}=$ $\left\|A\left(T_{0}\right) u\right\|_{L^{p}\left(0, T_{0} ; X\right)}$. Using inequalities (6.8), (6.11) and (6.3) we obtain

$$
\begin{gathered}
\|w\|_{L^{p}\left(0, \hat{T} ; X_{1}\right)} \leq M\left(\left\|f+\left(A\left(T_{0}\right)-A(t)\right) A\left(T_{0}\right)^{-1} v_{0}\right\|_{L^{p}(0, \hat{T} ; X)}+\left\|x_{0}\right\|_{Z_{0}}+\left\|x_{1}\right\|_{Z_{1}}\right) \\
\leq M\left(1+a_{2} a_{1}^{-1} \rho_{A, T}\left(T_{\varepsilon}\right) N\left(T_{0}\right)\right)\left(\|f\|_{L^{p}(0, T ; X)}+\left\|x_{0}\right\|_{Z_{0}}+\left\|x_{1}\right\|_{Z_{1}}\right)
\end{gathered}
$$

and (6.11) holds with $T_{0}$ replaced by $\hat{T}$ and $N\left(T_{0}\right)$ replaced by

$$
N(\hat{T})=M\left(1+a_{2} a_{1}^{-1} \rho_{A, T}\left(T_{\varepsilon}\right) N\left(T_{0}\right)\right) .
$$

Since this procedure can be repeated with the same $T_{\varepsilon}$, we find a solution on $[0, T]$ that satisfies the bound (6.6), where we have obtained by induction:

$$
N=M \sum_{i=0}^{k}\left(a_{2} a_{1}^{-1} \rho_{A, T}\left(T_{\varepsilon}\right) M\right)^{i},
$$

where $k \in \mathbb{N}$ is the necessary number of steps $k=\left\lfloor T / T_{\varepsilon}\right\rfloor+1$. Applying (6.9) it follows

$$
N \leq M \sum_{i=0}^{k} 2^{-i}<M \sum_{i=0}^{\infty} 2^{-i}=2 M .
$$


Suppose $(\boldsymbol{A 1})$ is fulfilled. It is interesting to see when $(\boldsymbol{A 2})$ together with continuity of $A(t)$ for $t \in[0, T]$ and the compactness of $[0, T]$ implies $(\boldsymbol{A 3})$. According to the theorems in Section 4.3, the constant of maximal regularity depends always on $K_{A(t)}(\theta)$, $\theta \in\left(\alpha \pi / 2, \pi-\omega_{A(t)}\right)$. Next lemma gives the uniform boundedness of these quantities for $t \in[0, T]$.

Lemma 6.3 Let $\{A(t)\}_{t \in[0, T]}$ be a family of operators satisfying $(\boldsymbol{A 1})$. Then there exist constants $\omega$ and $K$ such that $\omega_{A(t)}<\omega<\pi(1-\alpha / 2)$ and $K_{A(t)}(\pi-\omega) \leq K$ for any $t \in[0, T]$.

Proof: Clearly $(\boldsymbol{A 1})$ implies that given $s \in[0, T]$,

$$
\left\|(\lambda I+A(s))^{-1}\right\|_{\mathcal{B}(X)} \leq \frac{K_{s}}{1+|\lambda|}, \lambda \in \overline{\Sigma_{\phi_{s}}} \cup\{0\}
$$

where $\alpha \pi / 2<\phi_{s}<\phi_{A(s)}=\pi-\omega_{A(s)} \leq \pi$. Moreover,

$$
(\lambda I+A(t))^{-1}=(\lambda I+A(s))^{-1}\left[I+(A(t)-A(s)) A(0)^{-1} A(0)(\lambda I+A(s))^{-1}\right]^{-1}
$$

for $t \in[0, T]$ with $|t-s| \leq \delta_{s}$ and $\lambda \in \overline{\Sigma_{\phi_{s}}} \cup\{0\}$. So, due to the compactness of $[0, T]$ there are constants $K \geq 0$ and $\alpha \pi / 2<\phi \leq \pi$ such that

$$
\left\|(\lambda I+A(t))^{-1}\right\|_{\mathcal{B}(X)} \leq \frac{K}{1+|\lambda|}
$$

for $\lambda \in \overline{\Sigma_{\phi}} \cup\{0\}, t \in[0, T]$. To obtain the desired result we have to set only $\omega=\pi-\phi$. Since in the Hilbert space case the $\mathcal{R}$-boundedness is equivalent to the uniform boundedness, then the constant of maximal regularity depends only on $K_{A(t)}(\theta)$. Therefore, according to the above lemma, in this case $(\boldsymbol{A 1})$ together with $(\boldsymbol{A 2})$ implies $(\boldsymbol{A 3})$.

We are now ready to apply the results obtained so far in this section to the fractional Löwner-Kufarev equation (5.60) which can be rewritten in an abstract form as

$$
D_{t}^{\alpha} u+C(t) A u=0,\left(g_{1-\alpha} * u\right)(0)=u_{0} ; \quad \alpha \in(0,1),
$$

where $A$ and $C(t)$ are defined by (5.61) and (5.64), and $u=u(\theta, t)=\Omega\left(e^{i \theta}, t\right), u_{0}(\theta)=$ $\Omega\left(e^{i \theta}\right)$. Take as a basic space $H_{1 / 2}$, defined by (5.63). We have already proved that in this space the family of operators $A(t)=C(t) A$ satisfies $(\boldsymbol{A 1})$. According to Theorem 4.16, if $\alpha \in(1 / 2,1),(\boldsymbol{A 2})$ is also satisfied for the equation with $f \equiv 0$ and with $Z_{0}:=$ $\left(H_{1 / 2}, H_{3 / 2}\right)_{\frac{1}{2 \alpha}, 2}$. Next apply Lemma 6.3 to obtain $(\boldsymbol{A 3})$. Therefore Lemma 6.2 applies and gives:

Proposition 6.4 Let $\alpha \in(1 / 2,1)$ and the spaces $H_{1 / 2}$ and $H_{3 / 2}$ be defined as in Section 5.5. Assume that $F\left(e^{i \theta}, t\right)$ is continuous on $(\theta, t) \in[0,2 \pi] \times[0, T],(5.66)$ is satisfied and $\Omega_{0}\left(e^{i \theta}\right) \in\left(H_{1 / 2}, H_{3 / 2}\right)_{\frac{1}{2 \alpha}, 2}$. Then the fractional Löwner-Kufarev equation (5.60) has a unique solution $\Omega\left(e^{i \theta}, t\right) \in L^{2}\left(0, T ; H_{3 / 2}\right) \cap H^{\alpha, 2}\left(0, T ; H_{1 / 2}\right)$ and

$$
\|\Omega\|_{L^{2}\left(0, T ; H_{1 / 2}\right)}+\|\Omega\|_{L^{2}\left(0, T ; H_{3 / 2}\right)}+\|\Omega\|_{H^{\alpha, 2}\left(0, T ; H_{1 / 2}\right)} \leq C\left\|\Omega_{0}\right\|_{\left(H_{1 / 2}, H_{3 / 2}\right) \frac{1}{2 \alpha, 2}} \cdot
$$


Turn back to our abstract problem. For Banach spaces of class $\mathcal{H} \mathcal{T}$ the constant of maximal regularity depends also on $\mathcal{R}_{A(t)}(\alpha \pi / 2)$. So, in order to obtain $(\boldsymbol{A 3})$ we have to require the uniform boundedness of this quantity. Then Lemma 6.3 implies that if we apply Theorems 4.16 and 4.21 to problem $(6.4), s \in[0, T]$, where the family $\{A(t)\}$ satisfies $(\boldsymbol{A 1})$, then the estimates given in these theorems are uniform with respect to $s \in[0, T]$. Note that $D_{A(s)}(\delta, p)=D_{A}(\delta, p)$ because $D(A(s))=D(A)$. So, combining these results with Lemma 6.2 , we obtain the following theorem

Theorem 6.5 Suppose that $\alpha \in(0,2), 1<p<\infty, X$ is a Banach space of class $\mathcal{H} \mathcal{T}$, $\{A(t)\}_{t \in[0, T]}$ is a family of operators satisfying $(\boldsymbol{A 1})$ and $f \in L^{p}(I ; X)$. Let moreover $A(t)$ be $\mathcal{R}$-sectorial operators with $\mathcal{R}$-angle

$$
\omega_{A(t)}^{R}<\pi(1-\alpha / 2)
$$

and $\mathcal{R}_{A(t)}(\alpha \pi / 2) \leq \mathcal{R}$ for $t \in[0, T]$. Let one of the following conditions be satisfied

(a) $\alpha \in(0,1), 1<p<\frac{1}{1-\alpha}$ and $x_{0} \in D_{A}\left(\frac{p-1}{\alpha p}, p\right)$;

(b) $\alpha \in(0,1), p \geq \frac{1}{1-\alpha}$ and $x_{0}=0$;

(c) $\alpha \in(1,2), 1<p<\frac{1}{2-\alpha}, x_{0} \in D_{A}\left(\frac{2 p-1}{\alpha p}, p\right)$ and $x_{1} \in D_{A}\left(\frac{p-1}{\alpha p}, p\right)$;

(d) $\alpha \in(1,2), p \geq \frac{1}{2-\alpha}, x_{0}=0$ and $x_{1} \in D_{A}\left(\frac{p-1}{\alpha p}, p\right)$.

Then problem (6.1), (6.2) has an unique strict $L^{p}$ solution $u$ satisfying

$\|u\|_{L^{p}(I ; X)}+\left\|D_{t}^{\alpha} u\right\|_{L^{p}(I ; X)}+\|A u\|_{L^{p}(I ; X)} \leq C\left(\left\|x_{0}\right\|_{D_{A}\left(\frac{m p-1}{\alpha p}, p\right)}+\left\|x_{1}\right\|_{D_{A}\left(\frac{p-1}{\alpha p}, p\right)}+\|f\|_{L^{p}(I ; X)}\right)$,

where $m=\lceil\alpha\rceil$ and we set $x_{1} \equiv 0$ if $\alpha \in(0,1]$. The constant $C$ depends on $X, \alpha, p, \mathcal{R}, \omega$ and $K$, given in Lemma 6.3 , but does not depend on $T$ and on the individual operators $A(t)$.

Obviously, Lemma 6.2 is fulfilled if we take instead of the whole space $X$ some interpolation space $Y=D_{A}(\delta, p), \delta \in(0,1), 1<p<\infty$ and $Y_{1}:=\{u \in D(A): A u \in Y\}$, $\|u\|_{Y_{1}}:=\|A u\|_{Y}$. This observation together with Theorems 4.18 and 4.22 and Lemma 6.3 implies the following result:

Theorem 6.6 Suppose that $\alpha \in(0,2), 1<p<\infty, \delta \in(0,1),\{A(t)\}_{t \in[0, T]}$ is a family of operators in a Banach space $X$ satisfying $(\boldsymbol{A 1}), f \in L^{p}\left(0, T ; D_{A}(\delta, p)\right)$. Suppose that any of the following conditions is satisfied

(a) $\alpha \in(0,1), 1<p<\frac{1}{1-\alpha}, 0<\delta<\frac{\alpha p-p+1}{\alpha p}, x_{0} \in D_{A}\left(\frac{p-1}{\alpha p}+\delta, p\right)$;

(b) $\alpha \in(0,1), 1<p<\frac{1}{1-\alpha}, \frac{\alpha p-p+1}{\alpha p} \leq \delta<1, x_{0}=0$;

(c) $\alpha \in(0,1), p \geq \frac{1}{1-\alpha}, x_{0}=0$; 
(d) $\alpha \in(1,2), 1<p<\frac{1}{2-\alpha}, 0<\delta<\frac{\alpha p-2 p+1}{\alpha p}, x_{0} \in D_{A}\left(\frac{2 p-1}{\alpha p}+\delta, p\right), x_{1} \in D_{A}\left(\frac{p-1}{\alpha p}+\delta, p\right)$;

(e) $\alpha \in(1,2), 1<p<\frac{1}{2-\alpha}, \frac{\alpha p-2 p+1}{\alpha p} \leq \delta<\frac{\alpha p-p+1}{\alpha p}, x_{0}=0, x_{1} \in D_{A}\left(\frac{p-1}{\alpha p}+\delta, p\right)$;

(f) $\alpha \in(1,2), p \geq \frac{1}{2-\alpha}, 0<\delta<\frac{\alpha p-p+1}{\alpha p}, x_{0}=0, x_{1} \in D_{A}\left(\frac{p-1}{\alpha p}+\delta, p\right)$;

(g) $\alpha \in(1,2), p>1, \frac{\alpha p-p+1}{\alpha p} \leq \delta<1, x_{0}=0, x_{1}=0$.

Then (6.1), (6.2) is strictly solvable in $L^{p}\left(0, T ; D_{A}(\delta, p)\right)$ and the following estimate holds

$$
\begin{gathered}
\|u\|_{L^{p}\left(I ; D_{A}(\delta, p)\right)}+\left\|D_{t}^{\alpha} u\right\|_{L^{p}\left(I ; D_{A}(\delta, p)\right)}+\|A u\|_{L^{p}\left(I ; D_{A}(\delta, p)\right)} \\
\leq C\left(\left\|x_{0}\right\|_{D_{A}\left(\frac{m p-1}{\alpha p}+\delta, p\right)}+\left\|x_{1}\right\|_{D_{A}\left(\frac{p-1}{\alpha p}+\delta, p\right)}+\|f\|_{L^{p}\left(I ; D_{A}(\delta, p)\right)}\right),
\end{gathered}
$$

where $m=\lceil\alpha\rceil$ and $x_{1} \equiv 0$ if $\alpha \in(0,1]$. The constant $C$ depends on $X, \alpha, p, \delta, \omega$ and $K$, given in Lemma 6.3 , but does not depend on $T$ and on the individual operators $A(t)$.

\subsection{Global solutions for a quasilinear equation}

Let $\alpha \in(1,2), 1<p, q<\infty, T>0$. Suppose that

$$
\sigma \in C^{2}(\mathbb{R}) \text { and } 0<\sigma_{0} \leq \sigma^{\prime}(y) \leq \sigma_{1}<\infty, \quad y \in \mathbb{R}
$$

for some constants $\sigma_{0}, \sigma_{1}$. In this section we establish the global existence of a strict solution in $L^{p}\left(0, T ; L^{q}(0,1)\right)$ of the following quasilinear equation for $u=u(t, x)$ :

$$
D_{t}^{\alpha} u-\left(\sigma\left(u_{x}\right)\right)_{x}=f, \quad t>0, x \in(0,1)
$$

with Dirichlet boundary conditions

$$
u(t, 0)=u(t, 1)=0, t>0,
$$

and initial conditions

$$
\lim _{t \downarrow 0} \int_{0}^{t} g_{2-\alpha}(t-s) u(s, x) d s=0 ; \lim _{t \downarrow 0} \frac{d}{d t} \int_{0}^{t} g_{2-\alpha}(t-s) u(s, x) d s=0, \quad x \in(0,1) .
$$

The reason for studying this equation is that it and various variants of it appear in mathematical models of viscoelasticity. It is also of interest in itself when one explores the borderline between the quasilinear diffusion and quasilinear wave propagation. Equations of this type are considered in [43] and [31]. In the first paper the problem is studied in the Hilbert space setting under the assumption that $\sigma_{1} / \sigma_{0}$ is sufficiently small. In the second paper a very general $L^{p}\left(L^{q}\right)$ setting is considered and existence and uniqueness of a global solution is proved provided $\alpha<4 / 3$. We use a similar assumption as in [43] but a different approach, based on $L^{p}\left(L^{q}\right)$ estimates, to obtain global existence for $p, q$ large enough and all $\alpha \in(1,2)$. 
Denote by $c$ the mean of $\sigma_{0}$ and $\sigma_{1}$ :

$$
c:=\frac{\sigma_{0}+\sigma_{1}}{2}
$$

Let $W_{\#}^{2, q}(0,1):=\left\{u \in W^{2, q}(0,1) \mid u(0)=u(1)=0\right\}$ and

$$
E_{p, q}:=H_{0}^{\alpha, p}\left(0, T ; L^{q}(0,1)\right) \cap L^{p}\left(0, T ; W_{\#}^{2, q}(0,1)\right), \quad \alpha \neq 1+1 / p .
$$

We use also for shortness the following notations, where $v \in L^{p}\left(0, T ; W_{\#}^{2, q}(0,1)\right)$ :

$$
A u:=-c u_{x x}, \quad D(A)=W_{\#}^{2, q}(0,1) ; \quad M(v) u:=\frac{1}{c} \sigma^{\prime}\left(v_{x}\right) u, \quad D(M(v))=L^{q}(0,1) .
$$

Let $\mathcal{A}$ and $\mathcal{M}(v)$ be the extension of the above operators to $L^{p}\left(0, T ; L^{q}(0,1)\right)$, i.e. $\mathcal{A} \in$ $\mathcal{B}\left(L^{p}\left(0, T ; W_{\#}^{2, q}(0,1)\right), L^{p}\left(0, T ; L^{q}(0,1)\right)\right), \mathcal{M}(v) \in \mathcal{B}\left(L^{p}\left(0, T ; L^{q}(0,1)\right)\right)$. Denote as usual by $\mathcal{L}_{\alpha}$ the extension of $D_{t}^{\alpha}$ to $L^{p}\left(0, T ; L^{q}\right)$.

Our ultimate goal is to solve the problem

$$
\mathcal{L}_{\alpha} u+\mathcal{M}(u) \mathcal{A} u=f
$$

Consider first the linear autonomous equation

$$
\mathcal{L}_{\alpha} w+\mathcal{A} w=f_{0},
$$

on $\mathbb{R}$, where $f_{0}$ is the extension of $f$ with 0 outside $[0, T]$ and $\mathcal{L}_{\alpha}$ is the fractional derivative on $L^{p}\left(\mathbb{R} ; L^{q}(0,1)\right)$ defined as in the proof of Corollary 4.6. Since for any $\varepsilon>0, \mathcal{L}_{\alpha} \in$ $\mathcal{B I} \mathcal{P}\left(L^{p}\left(\mathbb{R} ; L^{q}(0,1)\right) ; \alpha \pi / 2+\varepsilon\right)$ ( [64], Theorem 8.6$)$ and $\mathcal{A} \in \mathcal{B I} \mathcal{P}\left(L^{p}\left(\mathbb{R} ; L^{q}(0,1)\right) ; \varepsilon\right.$ ) ( see e.g. [66] ) then $\left(\mathcal{L}_{\alpha}, \mathcal{A}\right)$ is a $\lambda$-regular pair of operators in $L^{p}\left(\mathbb{R} ; L^{q}(0,1)\right)$ (Corollary 4.12). Therefore, for any $f \in L^{p}\left(\mathbb{R} ; L^{q}(0,1)\right)$ there is a unique solution $w \in H_{0}^{\alpha, p}\left(\mathbb{R} ; L^{q}(0,1)\right) \cap$ $L^{p}\left(\mathbb{R} ; W_{\#}^{2, q}(0,1)\right)$, satisfying

$$
\left\|D_{t}^{\alpha} w\right\|_{L^{p}\left(\mathbb{R} ; L^{q}(0,1)\right)}+\|A w\|_{L^{p}\left(\mathbb{R} ; L^{q}(0,1)\right)} \leq C_{p, q}\|f\|_{L^{p}\left(\mathbb{R} ; L^{q}(0,1)\right)},
$$

where $C_{p, q}$ depends on $p$ and $q$, but does not depend on $c$. Then the solution of (6.20) on $[0, T]$ is a restriction of the solution on $\mathbb{R}$ to $[0, T]$ and therefore it satisfies

$$
\left\|D_{t}^{\alpha} w\right\|_{L^{p}\left(0, T ; L^{q}(0,1)\right)}+\|A w\|_{L^{p}\left(0, T ; L^{q}(0,1)\right)} \leq C_{p, q}\|f\|_{L^{p}\left(0, T ; L^{q}(0,1)\right)} .
$$

Next we show that if we take $p$ and $q$ large enough, then

$$
E_{p, q} \hookrightarrow C\left(0, T ; C^{1}(0,1)\right),
$$

where the embedding is compact. First, according to the so-called mixed derivative theorem ( see [68], ) for any $\theta \in(0,1)$,

$$
D\left(\mathcal{L}_{\alpha}\right) \cap D(\mathcal{A}) \subset D\left(\mathcal{L}_{\alpha}^{\theta} \mathcal{A}^{1-\theta}\right) .
$$


Since ( see $[70]$ )

$$
\begin{gathered}
D\left(\mathcal{L}_{\alpha}^{\theta}\right)=\left[H_{0}^{\alpha, p}\left(0, T ; L^{q}(0,1)\right), L^{p}\left(0, T ; L^{q}(0,1)\right)\right]_{\theta} \subseteq H^{\alpha \theta, p}\left(0, T ; L^{q}(0,1)\right), \\
D\left(\mathcal{A}^{1-\theta}\right)=\left[L^{p}\left(0, T ; W_{\#}^{2, q}(0,1)\right), L^{p}\left(0, T ; L^{q}(0,1)\right)\right]_{1-\theta} \subseteq L^{p}\left(0, T ; H^{2(1-\theta), q}(0,1)\right),
\end{gathered}
$$

then (6.24) implies

$$
E_{p, q} \hookrightarrow H^{\alpha \theta, p}\left(0, T ; H^{2(1-\theta), q}(0,1)\right)
$$

Suppose

$$
\frac{1}{\alpha p}+\frac{1}{2 q}<\frac{1}{2}
$$

Then $0<\frac{1}{\alpha p}<\frac{q-1}{2 q}<1$ and we can take $\theta \in\left(\frac{1}{\alpha p}, \frac{q-1}{2 q}\right)$. Therefore $0<\nu:=\alpha \theta-1 / p$, $1<\mu:=2(1-\theta)-1 / q$ and

$$
H^{\alpha \theta, p}\left(0, T ; H^{2(1-\theta), q}(0,1)\right) \hookrightarrow C^{\nu}\left(0, T ; C^{\mu}(0,1)\right) \hookrightarrow C\left(0, T ; C^{1}(0,1)\right) .
$$

The first embedding follows from Theorem 1.10, the second is compact and it is implied by the Arzelà-Ascoli theorem. Therefore the embedding (6.23) holds and it is compact.

Fix $p$ and $q$, satisfying (6.26) and let $C_{p, q}$ be the corresponding constant from (6.21) and (6.22). It follows from (6.27), continuity of $\sigma^{\prime}$ and (6.18) that $\mathcal{M}(u) \in C([0, T] \times[0,1])$ for $u \in E_{p, q}$. Moreover, (6.15) implies

$$
\|1-\mathcal{M}(u)\|_{C([0, T] \times[0,1])} \leq \frac{\sigma_{1}-\sigma_{0}}{\sigma_{1}+\sigma_{0}} .
$$

Take $\sigma_{0}$ and $\sigma_{1}$ such that

$$
\frac{\sigma_{1}}{\sigma_{0}}<\frac{C_{p, q}+1}{C_{p, q}-1}
$$

Then

$$
1>\delta_{p, q}:=\frac{\sigma_{1}-\sigma_{0}}{\sigma_{1}+\sigma_{0}} C_{p, q}
$$

Now we are ready to find a global solution of (6.19) applying the Schauder fixed point theorem to the equation

$$
\mathcal{L}_{\alpha} u+\mathcal{M}(v) \mathcal{A} u=f
$$

In what follows we denote for shortness $L^{p}\left(L^{q}\right):=L^{p}\left(0, T ; L^{q}(0,1)\right)$. We prove consequently

(a) for any $v \in E_{p, q}$ there exists a unique $u \in E_{p, q}$ satisfying (6.31). Denote the corresponding mapping $v \rightarrow u$ by $u=: G v ; G$ maps the closed convex set in $E_{p, q}$

$$
K:=\left\{u \in E_{p, q}:\left\|D_{t}^{\alpha} u\right\|_{L^{p}\left(L^{q}\right)}+\left(1-\delta_{p, q}\right)\|A u\|_{L^{p}\left(L^{q}\right)} \leq C_{p, q}\|f\|_{L^{p}\left(L^{q}\right)}\right\}
$$

into itself;

(b) $G: E_{p, q} \rightarrow E_{p, q}$ is a continuous mapping; 
(c) $G$ maps bounded sets in $E_{p, q}$ in relatively compact sets in $E_{p, q}$.

(a) We already have solvability and maximal regularity of the linear autonomous problem (6.20). The next step is to prove this for the equation

$$
\left(D_{t}^{\alpha} u\right)(t, x)+M(v(s, x)) A u(t, x)=f(t, x), \text { with fixed } s \in[0, T],
$$

where $v \in E_{p, q}$. Let

$$
L(0) u:=D_{t}^{\alpha} u+A u ; \quad L(1) u:=D_{t}^{\alpha} u+M(v(s, x)) A u .
$$

For $\rho \in[0,1]$, denote

$$
L(\rho):=(1-\rho) L(0)+\rho L(1) .
$$

The operators $L(0), L(1)$ are bounded linear operators from $E_{p, q}$ to $L^{p}\left(L^{q}\right)$. Moreover, there exists a constant $C^{\prime}>0$, independent of $\rho$, such that

$$
\|u\|_{E_{p, q}} \leq C^{\prime}\|L(\rho) u\|_{L^{p}\left(L^{q}\right)} .
$$

Indeed, let $L(\rho) u=f$, or, equivalently

$$
\left(D_{t}^{\alpha} u\right)(t, x)+A u(t, x)=f(t, x)+\rho(1-M(v(s, x))) A u(t, x) .
$$

Applying (6.22), (6.28) and (6.30), we obtain

$$
\begin{aligned}
\left\|D_{t}^{\alpha} u\right\|_{L^{p}\left(L^{q}\right)}+\|A u\|_{L^{p}\left(L^{q}\right)} & \leq C_{p, q}\|f\|_{L^{p}\left(L^{q}\right)}+\rho \frac{\sigma_{1}-\sigma_{0}}{\sigma_{1}+\sigma_{0}} C_{p, q}\|A u\|_{L^{p}\left(L^{q}\right)} \\
& \leq C_{p, q}\|f\|_{L^{p}\left(L^{q}\right)}+\delta_{p, q}\|A u\|_{L^{p}\left(L^{q}\right)}
\end{aligned}
$$

therefore

$$
\left\|D_{t}^{\alpha} u\right\|_{L^{p}\left(L^{q}\right)}+\left(1-\delta_{p, q}\right)\|A u\|_{L^{p}\left(L^{q}\right)} \leq C_{p, q}\|f\|_{L^{p}\left(L^{q}\right)},
$$

and so, (6.33) is satisfied with $C^{\prime}:=\frac{C_{p, q}}{1-\delta_{p, q}}$. Since $\left.L(0): E_{p, q} \rightarrow L^{p}\left(L^{q}\right)\right)$ is an isomorphism, applying [36], Theorem 5.2, it follows that $L(1): E_{p, q} \rightarrow L^{p}\left(L^{q}\right)$ is also an isomorphism. In this way we proved solvability and maximal regularity of the equation (6.32). Looking at the above proof we see that the maximal regularity constant does not depend on $s$. So, the family of operators $A(t)$ defined for $t \in[0, T]$ by

$$
A(t) u:=M(v(t, x)) A u, \quad D(A(t)):=W_{\#}^{2, q}(0,1),
$$

satisfies conditions $(\boldsymbol{A 1}),(\boldsymbol{A 2})$ and $(\boldsymbol{A 3})$ of the previous section. Therefore, equation (6.31) has maximal regularity with estimate (6.34) and (a) is proved.

(b) Let $v_{n} \rightarrow v$ in $E_{p, q}$ and take $u_{n}=G\left(v_{n}\right), u=G(v)$. Hence, equation (6.31) is satisfied also with $u$ replaced by $u_{n}$ and $v$ replaced by $v_{n}$. Therefore,

$$
D_{t}^{\alpha}\left(u_{n}-u\right)+M(v) A\left(u_{n}-u\right)=\left(M(v)-M\left(v_{n}\right)\right) A u_{n}
$$

and according to (6.34)

$$
\begin{aligned}
\left\|D_{t}^{\alpha}\left(u_{n}-u\right)\right\|_{L^{p}\left(L^{q}\right)} & +\left(1-\delta_{p, q}\right)\left\|A\left(u_{n}-u\right)\right\|_{L^{p}\left(L^{q}\right)} \\
& \leq C_{p, q}\left\|M(v)-M\left(v_{n}\right)\right\|_{C([0, T] \times[0,1])}\left\|A u_{n}\right\|_{L^{p}\left(L^{q}\right)} .
\end{aligned}
$$


By (6.34), $\left\|A u_{n}\right\|_{L^{p}\left(L^{q}\right)}$ is bounded by a constant, which does not depend on $n$. Since $v_{n} \rightarrow v$ in $E_{p, q}$ and because of the embedding (6.27) and the continuity of $\sigma^{\prime}$, it follows that

$$
\left\|M(v)-M\left(v_{n}\right)\right\|_{C([0, T] \times[0,1])}=\left\|\sigma^{\prime}\left(\left(v_{n}\right)_{x}\right)-\sigma^{\prime}\left(v_{x}\right)\right\|_{C([0, T] \times[0,1])} \rightarrow 0, \quad n \rightarrow \infty .
$$

So, the right-hand side of (6.35) tends to 0 as $n \rightarrow \infty$. Therefore $u_{n} \rightarrow u$ in $E_{p, q}$ and (b) is proved.

(c) Let $v_{n}$ be a bounded sequence in $E_{p, q}$. Since (6.23) is a compact embedding, there exists a subsequence $v_{n, k}$, convergent in $C\left(0, T ; C^{1}(0,1)\right)$. Therefore, (6.36) is satisfied with $v_{n}$ replaced by $v_{n, k}$. Applying now the same argument as in the proof of (b) (with $v_{n}$ replaced by $\left.v_{n, k}\right)$ we obtain that there exists $u \in E_{p, q}$, such that $G\left(v_{n, k}\right) \rightarrow u$ in $E_{p, q}$.

Properties (a), (b), and (c) imply that $G$ is a completely continuous operator which maps the bounded closed convex set $K$ into itself. According to the Schauder fixed point theorem there exists at least one function $u \in K$ such that $G u=u$ and this is a solution of (6.19). The uniqueness of the solution can be proved as in [31]. In this way we proved the global solvability of (6.16).

Theorem 6.7 Let $\alpha \in(1,2), T>0$ and $1<p, q<\infty$ are such that $\alpha \neq 1+1 / p$ and (6.26) hold. Let $\sigma_{0}, \sigma_{1}>0$ satisfy (6.29) and let the function $\sigma(y)$ yields (6.15). Then for any $f \in L^{p}\left(0, T ; L^{q}(0,1)\right)$ there exists a unique function $u \in H_{0}^{\alpha, p}\left(0, T ; L^{q}(0,1)\right) \cap$ $L^{p}\left(0, T ; W_{\#}^{2, q}(0,1)\right)$ satisfying $(6.16)$.

If $\alpha=1+1 / p$ then the above theorem also holds but we have to take $R_{0}^{\alpha, p}\left(0, T ; L^{q}(0,1)\right)$ instead of $H_{0}^{\alpha, p}\left(0, T ; L^{q}(0,1)\right)$.

Condition (6.26) shows that this approach is not applicable in the purely Hilbert space case $p=q=2$.

Implicit estimates for the constant $C_{p, q}$ could be obtained by the multiplier theorem or Dore-Venni theorem. 


\section{Bibliography}

[1] H. A m a n n, Linear and Quasilinear Parabolic Problems, Vol. 1. Birkhäuser Verlag, Basel, Boston, Berlin (1995).

[2] W. A r e n d t, O. E 1 M e n n a o u i, M. H i e b e r, Boundary values of holomorphic semigroups. Proc. Amer. Math. Soc. 125 No 3 (1997), 635-647.

[3] J. B. B a i 11 o n, Caractère borné de certains générateurs de semigroupes linéaires dans les espaces de Banach. C. R. Acad. Sci. Paris 290 (1980), 757-760.

[4] E. B a z h l e k o v a, Subordination principle for fractional evolution equations. Fractional Calculus \& Applied Analysis 3 No 3 (2000), 213-230.

[5] E. B a $\mathrm{z} \mathrm{h} \mathrm{l} \mathrm{e} \mathrm{k} \mathrm{o} \mathrm{v} \mathrm{a,} \mathrm{Perturbation} \mathrm{properties} \mathrm{for} \mathrm{abstract} \mathrm{evolution} \mathrm{equations}$ of fractional order. Transform Methods and Special Functions, AUBG'99. Fractional Calculus $\&$ Applied Analysis 2 No 4 (1999), 359-366.

[6] E. B a z h l e k o v a, The abstract Cauchy problem for the fractional evolution equation. Fractional Calculus \&f Applied Analysis 1 No 3 (1998), 255-270.

[7] E. $\mathrm{B} \mathrm{a} \mathrm{z} \mathrm{h} \mathrm{l} \mathrm{e} \mathrm{k} \mathrm{o} \mathrm{v}$ a, Perturbation and approximation properties for abstract evolution equations of fractional order, Research Report RANA 00-05, Eindhoven University of Technology, Eindhoven (2000).

[8] H. B r e z i s, Opérateurs Maximaux Monotones et Semi-groupes de Contractions dans les Espaces de Hilbert. Math. Studies 5, North-Holland, Amsterdam (1973).

[9] S. B u, S. G u e r r e-D e 1 a b r i e r e, Imaginary powers and interpolation spaces of unbounded operators. Rend. Istit. Mat. Univ. Trieste 28 No suppl. (1996), 91-100 (1997), Dedicated to the memory of Pierre Grisvard.

[10] M. C a p u t o, Linear models of dissipation whose $\mathrm{Q}$ is almost frequency independent, Part II. Geophys. J. R. Astr. Soc. 13 (1967), 529-539.

[11] M. C a p u t o, Elasticitá e Dissipazione. Zanichelli, Bologna (1969). (in Italian: Elasticity and Dissipation)

[12] M. C a p u t o, F. M a i n a r d i, Linear models of dissipation in anelastic solids. Riv. Nuovo Cimento (Ser. II) 1 (1971),161-198. 
[13] P h. C l é m e n t, On the method of sums of operators. Semi-groupes d'Opérateurs et Calcul Fonctionnel (Besançon, 1998) 1-30.

[14] P h. C l é m e n t, G. G r i p e n b e r g, V. H ö g n ä s, Some remarks on the method of sums. Stochastic Processes, Physics and Geometry; New Interplays, II (Lepzig, 1999) Amer. Math. Soc., Providence, RI (2000), 125-134

[15] P h. C l é m e n t, G. G r i p e n b e r g, S-O. L o n d e n, Schauder estimates for equations with fractional derivatives. Trans. Amer. Math. Soc. 352 No 5 (2000), 2239-2260.

[16] P h. C l é m e n t, G. G r i p e n b e r g, S-O. L o n d e n, Regularity properties of solutions of fractional evolution equations. Proc. 6-th International Conference on Evolution Equations, Bad Herrenalb 1998, to appear.

[17] P h. C 1 é m e n t, G. G r i p e n b e r g, S-O. L o n d e n, Hölder regularity for a linear fractional evolution equation. Topics in Nonlinear Analysis, The Herbert Amann Anniversary Volume, Birkhäuser, Basel (1998).

[18] P h. C l é m e n t, S.-O. L o n d e n, On the sum of fractional derivatives and $m$ -accretive operators. Partial Differential Equations / Models in Physics and Biology Mathematical Research 64, Academie Verlag Berlin (1994), 91-100.

[19] P h. C l é m e n t, E. M i t i d i e r i, Qualitative properties of solutions of Volterra equations in Banach spaces. Israel J. Math. 64 No 1 (1988), 1-24.

[20] P h. C l é m e n t, B. d e P a g t e r, F. A. S u k o c h e v, H. W i t v li e t, Schauder decompositions and multiplier theorems. Studia Math. 138 No 2 (2000), 135-163.

[21] P h. C l é m e n t, J. P r ü s s, Completely positive measures and Feller semigroups. Math. Ann. 287 (1990), 73-105.

[22] P h. C l é m e n t, J. P r ü s s, An operator-valued transference principle and maximal regularity on vector-valued $L^{p}$-spaces. Evolution Equations and Their Applications in Physical and Life Sciences (Bad Herrenalb, 1999) (2000), 67-88.

[23] G. D a $\mathrm{P} \mathrm{r}$ a t o, P. G r i s v a r d, Sommes d'opérateurs linéaires et équations différentielles opérationelles. J. Math. Pures Appl. 54 (1975), 305-387.

[24] G. D a P r a t o, M. I a n n e 11 i, Linear abstract integrodifferential equations of hyperbolic type in Hilbert spaces. Rend. Sem. Mat. Padova 62 (1980), 191-206.

[25] G. D a P r a t o, M. I a n n e $1 \mathrm{l}$ i, Linear integrodifferential equations in Banach spaces. Rend. Sem. Mat. Padova 62 (1980), 207-219.

[26] G. D a P r a t o, E. S i n e s t r a r i, Hölder regularity for nonautonomous abstract parabolic equations. Israel J. Math. 42 No 1-2 (1982), 1-19.

[27] G. D o r e, $L^{p}$-regularity for abstract differential equations, Functional Analysis and Related Topics, Proc. Kyoto (1991). 
[28] G. D o r e, A. V e n n i, On the closedness of the sum of two closed operators. Math. Z. 196 (1987), 189-201.

[29] M. M. D z r b a s h j a n, A. N e r s e s y a n, On the application of some integrodifferential operators. Dokl. Acad. Nauk 121 No 2 (1958), 210-213.

[30] P. E g b e r t s, On the Sum of Accretive Operators. Ph.D. Thesis, TU Delft (1992).

[31] H. E n g l e r, Global smooth solutions for a class of parabolic integro-differential equations. Trans. Amer. Math. Soc. 348 No 1 (1996), 267-290.

[32] A. E r d é l y i, W. M a g n u s, F. O b e r h e t t i n g e r, F. G. T r i c o m i, Higher Transcendental Functions. McGraw-Hill, New-York (1955).

[33] M. A. E v g r a f o v, Asymptotic Estimates and Entire Functions. Gordon and Breach (1961).

[34] H. O. F a t t o r i n i, Second Order Linear Differential Equations in Banach Spaces. North-Holland (1985).

[35] Y. F u j i t a, Integrodifferential equations which interpolate the heat equation and the wave equation. Osaka J. Math. 27 (1990), 309-321.

[36] D. G i 1 b a r g, N. S. T r u d i n g e r, Elliptic Partial Differential Equations of Second Order. Springer, Berlin (1983).

[37] J. G o l d s t e i n, Semigroups and second order differential equations, J. Functional Analysis, 4 (1969), 50-70.

[38] J. G o l d s t e i n, The universal addability problem for generators of cosine functions and operator groups. Houston J. Math. 6, No 3 (1980), 365-373.

[39] R. G o r e n f l o, Y u. L u c h k o, F. M a i n a r d i, Analytical properties and applications of the Wright function. Fractional Calculus \& Applied Analysis 2 No 4 (1999), 383-414.

[40] J. de G r a a f, Mathematical addenda to Hopper's model of plane Stokes flow driven by capillarity on a free surface. Geometric and Quantum Aspects of Integrable Systems (Scheveningen, 1992), 167-185, Lecture Notes in Phys., 424, Springer, Berlin (1993).

[41] J. de G r a a f, Evolution equations for polynomials and rational functions which are conformal on the unit disc. J. Comp. Appl. Math. (2001). Preprint in: Research Report RANA 99-39, Eindhoven University of Technology, Eindhoven (1999).

[42] G. G r i p e n b e r g, P h. C l é m e n t, S-O. L o n d e n, Smoothness in fractional evolution equations and conservation laws. Ann. Scuola Norm. Sup. Pisa Cl. Sci. 4 Vol.XXIX (2000), 231-251.

[43] G. G r i p e n b e r g, Global existence of solutions of Volterra integrodifferential equations of parabolic type. J. Diff. Eq. 102 (1993), 382-390. 
[44] P. G r i s v a r d, Équations différentielles abstraites. Ann. Sci. École Norm. Sup. (4) 2 (1969), 311-395.

[45] M. H i e b e r, Integrated semigroups and differential operators on $L^{p}$ spaces. Math. Ann. 291 (1991), 1-16.

[46] E. H i 1 l e, R. S. P h i 11 i p s, Functional Analysis and Semi-Groups. Amer. Math. Soc. Colloquium Pubs. 31 Providence (1957).

[47] N. K a l t o n, G. L a n c i e n, A solution to the problem of the $L^{p}$ maximal regularity, preprint (1999).

[48] T. K a t o, Perturbation Theory for Linear Operators. Springer-Verlag Berlin, Heidelberg, New-York (1966).

[49] T. K a t o, Fractional powers of dissipative operators. II J. Math. Soc. Japan 14 (1962), 242-248.

[50] T. K a t o, Remarks on pseudo-resolvents and infinitesimal generators of semi-groups. Proc. Japan Acad. 35 (1959), 467-468.

[51] B. K l e i n O b b i n k, Moving Boundary Problems in Relation with Equations of Löwner-Kufareev type. Ph.D. Thesis, TU Eindhoven, Eindhoven (1995).

[52] D. L u t z, On bounded time-dependent perturbations of operator cosine functions. Aequationes Math. 23 (1981), 197-203.

[53] F. M a i n a $\mathrm{r}$ d i, Fractional diffusive waves in viscoelastic solids. in: J.L. Wegner, F.R. Norwood (Eds.), IUTAM Symposium - Nonlinear Waves in Solids, ASME/AMR, Fairfield NJ (1995), 93-97. Abstract in: Appl. Mech. Rev. 46 (1993), 549.

[54] F. M a i n a r d i, Fractional relaxation-oscillation and fractional diffusion-wave phenomena. Chaos, Solitons and Fractals 7, No 9 (1996), 1461-1477.

[55] F. M a i n a r d i, Applications of Fractional Calculus in Mechanics. in: P. Rusev, I. Dimovski, V. Kiryakova (Eds.), Transform Methods and Special Functions, Varna 1996. SCTP, Singapore (1997), 309-334.

[56] F. M a i n a r d i, M. T o m i r o t t i, On a special function arising in the time fractional diffusion-wave equation. in: P. Rusev, I. Dimovski, V. Kiryakova (Eds.) Transform Methods and Special Functions, Sofia 1994. SCTP, Singapore (1995), 171-183.

[57] O. I. M a r i c h e v, Handbook of Integral Transforms of Higher Transcendental Functions: Theory and Applications. Ellis Horwood, Halsted Press (1983).

[58] K. S. M i 11 e r, B. R o s s, An Introduction to the Fractional Calculus and Fractional Differential Equations. Wiley, New York (1993). 
[59] S. M o n n i a u x, J. P r ü s s, A theorem of the Dore-Venni type for noncommuting operators. Trans. Amer. Math. Soc. 349 No 12 (1997), 4787-4814.

[60] B. N a g y, On cosine operator functions in Banach spaces. Acta Sci. Math. Szeged 36 (1974), 281-290.

[61] R. R. N i g m a t u $1 \mathrm{l}$ i n, The realization of the generalized transfer equation in a medium with fractal geometry. Phys. Stat. Sol. B 133 (1986), 425-430. [English translation from Russian]

[62] A. P a z y, Semigroup of Linear Operators and Applications to Partial Differential Equations. Applied Mathematical Sciences 44, Springer Verlag, New York (1983).

[63] I. P o d l u b n y, Fractional Differential Equations. Mathematics in Science and Engineering 198, Academic Press, New York (1999).

[64] J. P r ü s s, Evolutionary Integral Equations and Applications. Birkhäuser, Basel, Boston, Berlin (1993).

[65] J. P r ü s s, H. S o h r, On operators with bounded imaginary powers in Banach spaces. Math. Z. 203 (1990), 429-452.

[66] J. P r ü s s, H. S o h r, Imaginary powers of elliptic second order differential operators in $L^{p}$-spaces. Hiroshima Math. J. 23 (1993), 161-192.

[67] S. G. S a m k o, A. A. K i l b a s, O. I. M a r i c h e v, Integral and Derivatives of Fractional Order. Gordon Breach, New York (1993).

[68] P. E. S o b o l e v s k i i, Fractional powers of coercively positive sums of operators. Soviet Math. Dokl. 16 (1975).

[69] H. T a n a b e, Equations of Evolution. Pitman, London (1979).

[70] H. T r i e b e 1, Interpolation Theory, Function Spaces, Differential Operators. NorthHolland, Amsterdam (1978).

[71] H. F. T r o t t e r, On the product of semi-groups of operators. Proc. Amer. Math. Soc. 10 (1959), 545-551.

[72] L. W e i s, Operator-valued Fourier multiplier theorems and maximal $L^{p}$-regularity, preprint (1999).

[73] D. V. W i d d e r, The Laplace Transform. Princeton Univ. Press, Princeton (1946).

[74] E. M. W r i g h t, The generalized Bessel function of order greater than one. Quarterly Journal of Mathematics (Oxford ser.) 11 (1940), 36-48.

[75] A. Y a g i, Coincidence entre des espaces d'interpolation et des domaines de puissances fractionnaires d'opérateurs. C. R. Acad. Sci. Paris Sér.I Math. 299 No. 6 (1984), 173-176. 


\section{Index}

\begin{tabular}{|c|c|}
\hline$(X, Y)_{\gamma, p}, 9$ & $W_{\#}^{2, q}(0,1), 94$ \\
\hline$A_{G}, 49$ & {$[X, Y]_{\theta}, 18$} \\
\hline$A_{\lambda}, 74$ & {$[x]_{D_{A}(\gamma, p)}, 9$} \\
\hline$C(I ; X), 6$ & $\mathcal{A}^{\alpha}, 25$ \\
\hline$C^{\lambda}(I ; X), 7$ & $\mathcal{B}(X), 5$ \\
\hline$C^{m}(I ; X), 6$ & $\mathcal{B}(X, Y), 5$ \\
\hline$C_{0}^{\infty}(\mathbb{R} ; X), 6$ & $\mathcal{B I} \mathcal{P}, 9$ \\
\hline$C_{p, q}, 94$ & $\mathbb{C}, 5$ \\
\hline$D(A), 5$ & $\mathcal{C}^{\alpha}, 20$ \\
\hline$D_{A}(\gamma, p), 9$ & $\Gamma_{r, \theta}, 62$ \\
\hline$D_{t}^{\alpha}, 10$ & $\mathbb{N}, 5$ \\
\hline$D_{t}^{m}, 10$ & $\mathbb{N}_{0}, 5$ \\
\hline$E_{\alpha, \beta}(z), 12$ & $\Phi_{\gamma}(z), 13$ \\
\hline$E_{\alpha}(z), 12$ & $\mathbb{R}, 5$ \\
\hline$E_{p, q}, 94$ & $\mathbb{R}_{+}, 5$ \\
\hline$G_{\gamma}, 48$ & $\mathcal{R}_{A}(\theta), 59$ \\
\hline$H^{\alpha, p}(I ; X), 18$ & $\Sigma_{\theta}, 5$ \\
\hline$H^{\alpha, p}(\mathbb{R} ; X), 18$ & $\Sigma_{\theta}(\omega), 5$ \\
\hline$H_{0}^{\alpha, p}(I ; X), 18$ & $*, 6$ \\
\hline$J_{t}^{\alpha}, 10$ & $\mathbf{D}_{t}^{\alpha}, 11$ \\
\hline$K_{A}(\phi), 8$ & $\hookrightarrow, 5$ \\
\hline$L^{p}(I ; X), 6$ & $\lceil\alpha\rceil, 5$ \\
\hline$L^{p}\left(\mathbb{R}_{+}, e^{-t} d t\right), 48$ & $\lfloor\alpha\rfloor, 5$ \\
\hline$L^{2,+}(0,2 \pi ; \mathbb{C}), 84$ & $\omega_{A}, 8$ \\
\hline$N(A), 5$ & $\varphi_{t, \gamma}(s), 40$ \\
\hline$R(A), 5$ & $\phi_{A}, 8$ \\
\hline$R(\lambda, A), 5$ & $\sigma(A), 5$ \\
\hline$R^{\alpha, p}(I ; X), 15$ & $\theta_{A}, 9$ \\
\hline$R_{0}^{\alpha, p}(I ; X), 15$ & $\varrho(A), 5$ \\
\hline$S_{\alpha}(t), 20$ & $g_{\beta}(t), 10$ \\
\hline$S_{\alpha}(t ; A), 33$ & $h^{\gamma}(I ; X), 7$ \\
\hline$W^{1,2,+}(0,2 \pi ; \mathbb{C}), 84$ & \\
\hline$W^{\beta, p}(I ; X), 18$ & $\mathcal{R}$-bound, 54 \\
\hline$W^{m, p}(I ; X), 7$ & angle \\
\hline$W_{0}^{m, p}(I ; X), 7$ & $\mathcal{R}, 59$ \\
\hline$W_{0}^{\beta, p}(I ; X), 18$ & power, 9 \\
\hline$W_{0}^{m, p}\left(\mathbb{R}_{+}, e^{-t} d t\right), 50$ & spectral, 8 \\
\hline
\end{tabular}


COF, 20

equation

fractional diffusion-wave, 27, 43

fractional oscillation, 13

fractional relaxation, 13

Löwner-Kufarev, 83

family

$\mathcal{R}$-bounded, 54

cosine operator, 20

formula

abstract Weierstrass, 39

Post-Widder inversion, 14

variation of parameters, 37

fractional derivative

Caputo, 11

Riemann-Liouville, 10

fractional integral, 10

function

completely monotonic, 13

Mittag-Leffler, 12

of Wright type, 13

inequality

generalized Minkowski, 64

Hölder, 6

Young, 6

kernel of positive type, 17,81

maximal $L^{p}$ regularity, 53, 55

operator

$\mathcal{R}$-sectorial, 59

$m$-accretive, 8, 72

accretive, 8

monotone, 72

nonnegative, 7

positive, 8

regularly $m$-accretive, 82

sectorial, 9

solution, 20

analytic, 25

exponentially bounded, 20

with bounded imaginary powers, 9

operators $\lambda$-regular pair of, 60

of subordination, 48

semigroup

$C_{0}, 20$

analytic, 25

boundary values of, 44

solution

strict $L^{p}, 61,88$

strong, 19

space

Bessel potential, 18

Hölder, 6

interpolation

complex, 18

real, 9

of class $\mathcal{H} \mathcal{T}, 9$

Sobolev, 7

of fractional order, 18

theorem

mixed derivative, 94

Schauder fixed point, 95

transform

Fourier, 7

Laplace, 7

Mellin, 45

well-posed problem, 19 


\section{Curriculum Vitae}

Emilia Bajlekova was born on May 12, 1963 in Pleven, Bulgaria. After finishing the secondary school of Mathematics, Pleven, from September 1981 till Jun 1986 she studied Mathematics at Sofia University "St. Kliment Ochridski", where she obtained her Master of Science degree in Complex Analysis. The graduation was followed by one year Post M.Sc. specialization at the same university. In the academic year 1990/1991 she was a part-time assistant in Analysis at the Faculty of Physics of this university. From January 1990 till May 1993 she was a Ph.D. student at the Institute of Mathematics and Informatics, Bulgarian Academy of Sciences. Later, from April 1995 till January 1997 she was appointed as a mathematician at the same institute. From December 1997 till September 2001 she carried out a doctoral research project at the Department of Mathematics and Computer Science, Eindhoven University of Technology. 


\section{THESES}

accompanying the dissertation

\section{Fractional Evolution Equations in Banach Spaces}

Emilia Grigorova Bajlekova

\section{I}

The fractional diffusion-wave equation interpolates between diffusion and wave propagation describing an intermediate process $(1<\alpha<2)$, or extrapolates from diffusion to a corresponding ultraslow process $(0<\alpha<1)$.

Section 2.2 of this thesis.

\section{II}

Let $A$ be a nonnegative operator in a Banach space $X, 0<\theta<1,1<p<\infty$, and $\alpha:=\frac{p-1}{\theta p}<2$. Let $P_{\alpha}(t)$ be the solution operator defined in Section 4.2. Then

$$
\|x\|_{\theta, p}:=\|x\|_{X}+\left\|A P_{\alpha}(t) x\right\|_{L^{p}(0, \infty ; X)}
$$

is an equivalent norm in the real interpolation space $(X, D(A))_{\theta, p}$. Section 4.3 of this thesis.

\section{III}

Maximal regularity is an important tool in treating quasi-linear equations. It enters usually in setting up a fixed-point argument.

Chapter 6 of this thesis.

\section{IV}

For generators $A$ of solution operators $S_{\alpha}(t)$ with arbitrary $\alpha>0$ several LandauKolmogorov-type inequalities hold. For example, if $A \in \mathcal{C}^{\alpha}(1,0)$, then

$$
\|A x\|^{2} \leq \frac{4 \alpha \Gamma^{2}(\alpha)}{\Gamma(2 \alpha)}\left\|A^{2} x\right\|\|x\| ; \quad\|A x\|^{3} \leq \frac{243 \alpha^{2} \Gamma^{3}(\alpha)}{4 \Gamma(3 \alpha)}\left\|A^{3} x\right\|\|x\|^{2},
$$

for $x \in D\left(A^{2}\right)$ and $x \in D\left(A^{3}\right)$, respectively.

See Section 2.1 of this thesis for notations. 
The Duhamel-type representation $u=\Omega * f$ of the solution $u(x, t)$ of an evolution equation, where $\Omega(x, t)$ is a kernel associated with the equation, $f(x, t)$ represents the initial data, $*$ is a convolution in the sense of [1], is appropriate for numerically solving the problem with various initial data.

[1] I.H. Dimovski, Convolutional Calculus. Kluwer, Dordrecht (1990).

[2] E. Bazhlekova, Duhamel-type representations of the solutions of non-local boundary value problems for the fractional diffusion-wave equation, in: P. Rusev, I. Dimovski, V. Kiryakova (Eds.), Transform Methods and Special Functions, Varna'96, Bulgarian Acad. Sci., Sofia (1998), pp. 32-40.

\section{VI}

The possible direct connection between Fractional Calculus and Fractals is rather actual but yet an open problem.

[1] B.B. Mandelbrot, The Fractal Geometry of Nature. Freeman, New York (1982).

[2] A. Carpinteri, F. Mainardi (Eds.), Fractals and Fractional Calculus in Continuum Mechanics. Springer Verlag, Wien (1997).

\section{VII}

Fractional Calculus is a "laboratory" for special functions and integral transforms. V. Kiryakova, All the special functions are fractional differintegrals of elementary functions. J.Physics A: Math.\& Gen. 30 No 14 (1997) pp. 5085-5103.

\section{VIII}

"There are two essential elements to teaching science. One is to know the subject. The other, more subtle and more difficult, is that you have to be able to remember what it was like not to understand something you now understand. That's very hard because each thing you understand transforms you for life. Nevertheless, the key to teaching that thing is to remember your untransformed self."

D. Goodstein, Needed: An Isaac Newton of Science education. Los Angeles Times, 3 Dec. 1989, M8.

\section{IX}

"How's your Mathematics? - I speak it like a native."

S. Milligan, The Goon Show Scripts. The Woburn Press, London (1972).

$\mathrm{X}$

We are independent: nothing depends on us. 